\title{
A Cyclopropanol-Based Strategy for Subunit Coupling: Total Synthesis of (+)- Spirolaxine Methyl Ether
}

\author{
Katie A. Keaton and Andrew J. Phillips* \\ Department of Chemistry and Biochemistry, University of Colorado, Boulder, CO 80309-0215
}

\section{SUPPORTING INFORMATION}

\section{General Experimental Procedures.}

${ }^{1} \mathrm{H}$ and ${ }^{13} \mathrm{C}$ NMR spectra were recorded at $25{ }^{\circ} \mathrm{C}$ on a Varian Inova spectrometer at 500 and $125 \mathrm{MHz}$, respectively, using $\mathrm{CDCl}_{3}$ as the solvent and internal reference. Coupling constants are reported in hertz, $\mathrm{Hz}$. All non-aqueous reactions were run in flame-dried glassware under a dry $\mathrm{N}_{2}$ atmosphere. Toluene, THF, $\mathrm{CH}_{2} \mathrm{Cl}_{2}$, and $\mathrm{Et}_{2} \mathrm{O}$ were obtained from Aldrich (Pure-Pac) and further dried by passage through activated alumina as described by Bergman and Grubbs. ${ }^{1}$ All flash chromatography was performed with

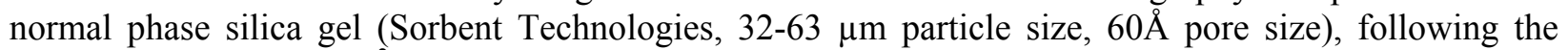
general protocol of Still. ${ }^{2}$ The $\mathrm{Ti}(\mathrm{i}-\mathrm{OPr})_{4}$ was distilled and diluted with dry, deoxygenated, hexanes to afford a $1 \mathrm{M}$ solution. Grignard reagents were freshly prepared and stored under $\mathrm{N}_{2(\mathrm{~g})}$, in the dark at room temperature. The $\mathrm{Fe}\left(\mathrm{NO}_{3}\right)_{3} \cdot 9 \mathrm{H}_{2} \mathrm{O}$ was obtained from Alpha-Aesar. The solid was dissolved in DMF (1M solution) and dried over $4 \AA$ molecular sieves for $16 \mathrm{~h}$ prior to use.

\section{Representative Kulinkovich Procedure}

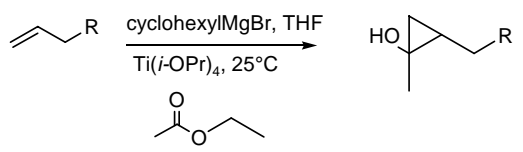

$\mathrm{Ti}(\mathrm{i}-\mathrm{OPr})_{4}(0.44 \mathrm{~mL}$ of a $1.0 \mathrm{M}$ solution in hexanes, $0.44 \mathrm{mmol}, 1.0 \mathrm{eq})$ was added to a flame dried flask

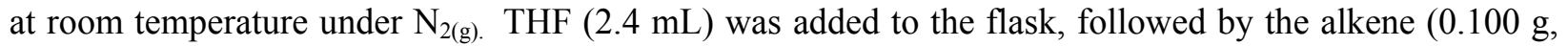
$0.44 \mathrm{mmol}, 1.0 \mathrm{eq})$ in THF $(2 \mathrm{~mL})$ and EtOAc $(0.064 \mathrm{~mL}, 0.65 \mathrm{mmol}, 1.5 \mathrm{eq})$. Then cyclohexylMgBr $\left(1.03 \mathrm{~mL}\right.$ of a $1.77 \mathrm{M}$ solution in $\left.\mathrm{Et}_{2} \mathrm{O}, 1.76 \mathrm{mmol}, 4 \mathrm{eq}\right)$ was added by syringe pump over the period of 1 $\mathrm{h}$ at $25^{\circ} \mathrm{C}$. The reaction was stirred for $1 \mathrm{~h}$ at $25^{\circ} \mathrm{C}$, diluted with EtOAc $(50 \mathrm{~mL})$ and poured into $\mathrm{NH}_{4} \mathrm{Cl}$ $(50 \mathrm{~mL})$. The mixture was stirred vigorously for $0.5 \mathrm{~h}$ to break up the emulsion and then filtered through celite. The layers were separated and the aqueous layer was extracted twice with EtOAc $(50 \mathrm{~mL} \times 2)$. The organic layers were combined, dried with $\mathrm{Na}_{2} \mathrm{SO}_{4}$, filtered and concentrated under reduced pressure. The residue was purified by flash column chromatography.

\footnotetext{
1 a) Alaimo, P. J.; Peters, D.W.; Arnold, J.; Bergman, R.G. J. Chem. Educ. 200178 64. b) Pangborn, A. B.; Giardello, M. A.; Grubbs, R. H.; Rosen, R.K.; Timmers, F. J. Organometallics 1996, 15, 151.

${ }^{2}$ Still, W.C.; Kahn, M.; Mitra, A. J. Org. Chem. 1978, 43, 2923.
} 


\section{Representative Ring Opening Procedure}

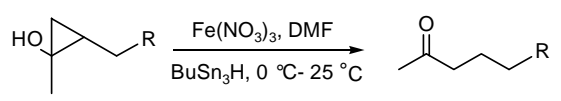

$\mathrm{Bu}_{3} \mathrm{SnH}(0.18 \mathrm{~mL}, 0.66 \mathrm{mmol}, 2$ eq.) was added to the cyclopropanol $(0.090 \mathrm{~g}, 0.33 \mathrm{mmol}, 1 \mathrm{eq})$ in DMF $(0.93 \mathrm{~mL})$ at $0{ }^{\circ} \mathrm{C}$ in a flame dried flask under $\mathrm{N}_{2(\mathrm{~g})}$. $\mathrm{Fe}\left(\mathrm{NO}_{3}\right)_{3}(0.73 \mathrm{~mL}, 0.73 \mathrm{mmol}, 1 \mathrm{M}$ solution in DMF, $2.2 \mathrm{eq}$ ) was then added over $30 \mathrm{~min}$ via syringe pump to the cooled solution. The reaction was stirred for $30 \mathrm{~min}$ at $0{ }^{\circ} \mathrm{C}$ and then warmed to room temperature and stirred for $1 \mathrm{~h}$. Upon completion the reaction was quenched with $\mathrm{H}_{2} \mathrm{O}(50 \mathrm{~mL})$. The aqueous layer was extracted with EtOAc $(20 \mathrm{~mL} \times 2)$ and the organic layers were combined, washed with saturated aqueous $\mathrm{NaCl}(20 \mathrm{~mL})$, dried with $\mathrm{MgSO}_{4}$, filtered and concentrated under reduced pressure. The residue was purified by flash column chromatography.

6-(tert-Butyl-dimethyl-silanyloxy)-7-methyl-octan-2-one (3):

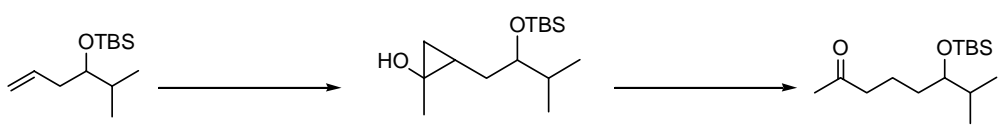

a) 2-[2-(tert-Butyl-dimethyl-silanyloxy)-3-methyl-butyl]-1-methyl-cyclopropanol, 2

${ }^{1} \mathrm{H}$ NMR $\left(400 \mathrm{MHz}, \mathrm{CDCl}_{3}\right) \delta 3.51-3.54(\mathrm{~m}, 1 \mathrm{H}), 1.76-1.84(\mathrm{~m}, 1 \mathrm{H}), 1.55-1.60(\mathrm{~m}, 1 \mathrm{H}), 1.45-1.51(\mathrm{dt}$, $1 \mathrm{H}, J=5.8,11.6 \mathrm{~Hz}), 1.41$ (s, 3H, major) 1.40 (minor), 1.15-1.25 (m, 1H), 1.02-1.08 (m, 1H), $0.89(\mathrm{~s}$, 9H), 0.80-0.89 (m, 6H), 0.07- $0.13(\mathrm{~m}, 1 \mathrm{H}), 0.049$ (s, 6H, 2x $\left.\mathrm{CH}_{3}\right)$.

b) 6-(tert-Butyl-dimethyl-silanyloxy)-7-methyl-octan-2-one, 3

${ }^{1} \mathrm{H}$ NMR $\left(500 \mathrm{MHz}, \mathrm{CDCl}_{3}\right) \delta 3.42(\mathrm{q}, 1 \mathrm{H}, J=5.6 \mathrm{~Hz}), 2.41(\mathrm{t}, 2 \mathrm{H}, J=7.6 \mathrm{~Hz}), 2.13(\mathrm{~s}, 3 \mathrm{H}), 1.68-1.74$ $(\mathrm{m}, 1 \mathrm{H}), 1.59-1.66(\mathrm{~m}, 1 \mathrm{H}), 1.49-1.57(\mathrm{~m}, 1 \mathrm{H}), 1.34-1.38(\mathrm{~m}, 2 \mathrm{H}), 0.88(\mathrm{~s}, 9 \mathrm{H}), 0.85(\mathrm{~d}, 3 \mathrm{H}, J=6.8)$, $0.83(\mathrm{~d}, 3 \mathrm{H}, J=6.8), 0.030(\mathrm{~s}, 3 \mathrm{H}), 0.025(\mathrm{~s}, 3 \mathrm{H}) ;{ }^{13} \mathrm{C} \mathrm{NMR}\left(400 \mathrm{MHz}, \mathrm{CDCl}_{3}\right) \delta 209.06,76.52,43.99$, $32.58,32.45,29.77,25.90,19.89,18.11,17.95,17.67,-4.32,-4.52$; IR (thin film) 2929, 2957, 2856, $1719,1472,1360,1251,1161,1051,835,665 \mathrm{~cm}^{-1}$; HRMS (EI) calculated for $\mathrm{C}_{15} \mathrm{H}_{32} \mathrm{O}_{2} \mathrm{SiNa}^{+}\left[\mathrm{M}+\mathrm{Na}^{+}\right]=$ 295.206378, found $295.206508 \pm<1.0 \mathrm{ppm}$.

6-(tert-Butyl-dimethyl-silanyloxy)-8-phenyl-octan-2-one (Table 1, entry 1):

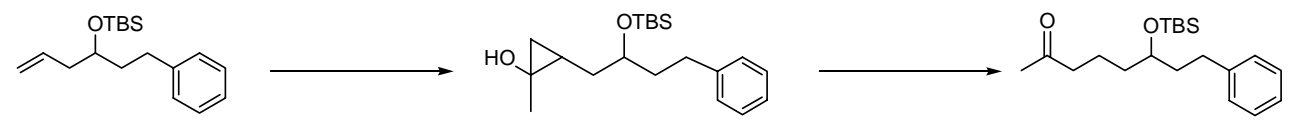

a) 2-[2-(tert-Butyl-dimethyl-silanyloxy)-4-phenyl-butyl]-1-methyl-cyclopropanol

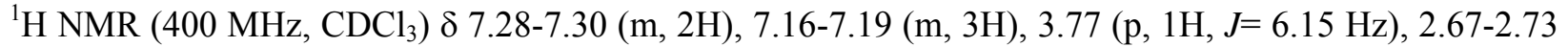
$(\mathrm{m}, 1 \mathrm{H}), 2.57-2.64(\mathrm{~m}, 1 \mathrm{H}), 1.82-1.91(\mathrm{~m}, 1 \mathrm{H}), 1.74-1.79(\mathrm{~m}, 1 \mathrm{H}), 1.61-1.71(\mathrm{~m}, 1 \mathrm{H}), 1.41(\mathrm{~s}, 3 \mathrm{H}), 1.18-$ $1.31(\mathrm{~m}, 1 \mathrm{H}), 1.02-1.10(\mathrm{~m}, 1 \mathrm{H}), 0.92(\mathrm{~s}, 9 \mathrm{H}), 0.86-0.89(\mathrm{~m}, 1 \mathrm{H}), 0.89-0.14(\mathrm{~m}, 1 \mathrm{H}), 0.074(\mathrm{~s}, 3 \mathrm{H})$, $0.066(\mathrm{~s}, 3 \mathrm{H})$. 
b) 6-(tert-Butyl-dimethyl-silanyloxy)-8-phenyl-octan-2-one

${ }^{1} \mathrm{H}$ NMR $\left(500 \mathrm{MHz}, \mathrm{CDCl}_{3}\right) \delta$ 7.28-7.30 (m, 2H), 7.16-7.19 (m, 3H), $3.72(\mathrm{p}, 1 \mathrm{H}, J=5.7 \mathrm{~Hz}), 2.56-2.70$ $(\mathrm{m}, 2 \mathrm{H}), 2.43(\mathrm{t}, 2 \mathrm{H}, J=7.5 \mathrm{~Hz}), 2.14(\mathrm{~s}, 3 \mathrm{H}), 1.73-1.78(\mathrm{~m}, 2 \mathrm{H}), 1.58-1.65(\mathrm{~m}, 2 \mathrm{H}), 1.45-1.50(\mathrm{~m}, 2 \mathrm{H})$, $0.91(\mathrm{~s}, 9 \mathrm{H}), 0.060(\mathrm{~s}, 3 \mathrm{H}), 0.047(\mathrm{~s}, 3 \mathrm{H}) ;{ }^{13} \mathrm{C} \mathrm{NMR}\left(400 \mathrm{MHz}, \mathrm{CDCl}_{3}\right) \delta 208.83,142.53,128.28$, $125.62,71.51,43.76,38.82,36.25,31.59,29.76,25.87,19.54,18.07,-4.44,-4.47$; IR (thin film) 2952, 2929, 2856, 1718, 1471, 1360, 1255, 1063, 1095, 1005, 835, 774, $699 \mathrm{~cm}^{-1}$; HRMS (EI) calculated for $\mathrm{C}_{20} \mathrm{H}_{34} \mathrm{O}_{2} \mathrm{SiNa}^{+}\left[\mathrm{M}+\mathrm{Na}^{+}\right]=357.222028$, found $357.222223 \pm<1.0 \mathrm{ppm}$.

\section{6-Triisopropylsilanyloxy-hexan-2-one (Table 1, entry 2):}

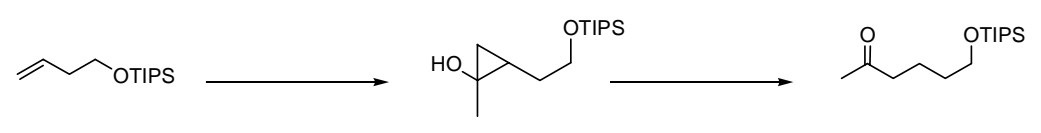

a) 1-Methyl-2-(2-triisopropylsilanyloxy-ethyl)-cyclopropanol

${ }^{1} \mathrm{H}$ NMR $\left(400 \mathrm{MHz}, \mathrm{CDCl}_{3}\right) \delta 3.73(\mathrm{t}, 2 \mathrm{H}, J=7.1 \mathrm{~Hz}), 1.61-1.69(\mathrm{~m}, 1 \mathrm{H}), 1.42(\mathrm{~s}, 3 \mathrm{H}), 1.36(\underline{?}, 1 \mathrm{H}, J=$ $6.6 \mathrm{~Hz}), 1.04-1.16(\mathrm{~m}, 22 \mathrm{H}), 0.84-0.88(\mathrm{~m}, 1 \mathrm{H}), 0.12(\mathrm{dd}, 1 \mathrm{H}, J=5.2,1.1 \mathrm{~Hz})$.

b) 6-Triisopropylsilanyloxy-hexan-2-one

${ }^{1} \mathrm{H}$ NMR $\left(500 \mathrm{MHz}, \mathrm{CDCl}_{3}\right) \delta 3.68(\mathrm{t}, 2 \mathrm{H}, J=6.4 \mathrm{~Hz}), 2.47(\mathrm{t}, 2 \mathrm{H}, J=7.4 \mathrm{~Hz}), 2.14(\mathrm{~s}, 3 \mathrm{H}), 1.63-1.69$ $(\mathrm{m}, 2 \mathrm{H}), 1.51-1.56(\mathrm{~m}, 2 \mathrm{H}), 1.05(\mathrm{~d}, 18 \mathrm{H}, J=4.8 \mathrm{~Hz}), 1.12-1.00(\mathrm{~m}, 3 \mathrm{H}) ;{ }^{13} \mathrm{C}$ NMR $\left(400 \mathrm{MHz}, \mathrm{CDCl}_{3}\right)$ $\delta$ 209.02, 62.95, 43.47, 32.27, 29.70, 20.31, 17.94, 11.88; IR (thin film) 2942, 2866, 1719, 1463, 1365, $1161,1107,995,882 \mathrm{~cm}^{-1}$; HRMS (EI) calculated for $\mathrm{C}_{15} \mathrm{H}_{32} \mathrm{O}_{2} \mathrm{SiNa}^{+}\left[\mathrm{M}+\mathrm{Na}^{+}\right]=295.206378$, found $295.206373 \pm<1.0 \mathrm{ppm}$.

6-(tert-Butyl-dimethyl-silanyloxy)-7-methyl-non-7-en-2-one (Table 1, entry 3):

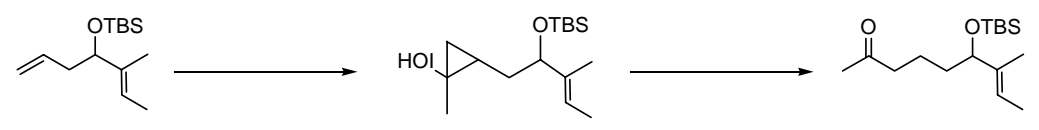

a) 2-[2-(tert-Butyl-dimethyl-silanyloxy)-3-methyl-pent-3-enyl]-1-methyl-cyclopropanol

${ }^{1} \mathrm{H}$ NMR (400 MHz, $\left.\mathrm{CDCl}_{3}\right) \delta 5.38(\mathrm{q}, 1 \mathrm{H}, J=6.2 \mathrm{~Hz}), 3.99(\mathrm{q}, 1 \mathrm{H}, J=7.1 \mathrm{~Hz}), 1.63-1.69(\mathrm{~m}, 1 \mathrm{H}), 1.54-$ $1.59(\mathrm{~m}, 6 \mathrm{H}), 1.40(\mathrm{~s}, 3 \mathrm{H}), 1.11-1.16(\mathrm{~m}, 1 \mathrm{H}), 0.98-1.03(\mathrm{~m}, 1 \mathrm{H}$, minor $\delta 0.93-0.98), 0.88(\mathrm{~s}, 9 \mathrm{H}), 0.80-$ $0.85(\mathrm{~m}, 1 \mathrm{H}), 0.066(\mathrm{dd}, 1 \mathrm{H}, J=0.10$, minor $\delta 0.10), 0.032(\mathrm{~s}, 3 \mathrm{H}),-0.020(\mathrm{~s}, 3 \mathrm{H})$.

b) 6-(tert-Butyl-dimethyl-silanyloxy)-7-methyl-non-7-en-2-one

${ }^{1} \mathrm{H}$ NMR $\left(400 \mathrm{MHz}, \mathrm{CDCl}_{3}\right) \delta 5.35(\mathrm{q}, 1 \mathrm{H}, J=5.8 \mathrm{~Hz}), 3.92(\mathrm{t}, 1 \mathrm{H}, J=5.8 \mathrm{~Hz}), 2.41(\mathrm{t}, 2 \mathrm{H}, J=6.8), 2.12$ (s, 3H), $1.56(\mathrm{~d}, 3 \mathrm{H}, J=6.8 \mathrm{~Hz}), 1.52(\mathrm{~s}, 3 \mathrm{H}), 1.38-1.49(\mathrm{~m}, 4 \mathrm{H}), 0.85(\mathrm{~s}, 9 \mathrm{H}), 0.0075(\mathrm{~s}, 3 \mathrm{H}),-0.039(\mathrm{~s}$, $3 \mathrm{H}) ;{ }^{13} \mathrm{C}$ NMR $\left(400 \mathrm{MHz}, \mathrm{CDCl}_{3}\right) \delta 209.10,137.92,119.78,78.31,43.66,35.58,29.76,25.80,20.23$, 18.25, 12.87, 10.64, -4.74, -5.10; IR (thin film) 2955, 2929, 2857, 1719, 1472, 1360, 1254, 1065, 836, $775 \mathrm{~cm}^{-1}$; HRMS (EI) calculated for $\mathrm{C}_{16} \mathrm{H}_{32} \mathrm{O}_{2} \mathrm{SiNa}^{+}\left[\mathrm{M}+\mathrm{Na}^{+}\right]=307.206378$, found $307.206321 \pm<1.0$ ppm.

\section{6-(tert-Butyl-dimethyl-silanyloxy)-5-methyl-8-phenyl-octan-2-one (Table 1, entry 4):}




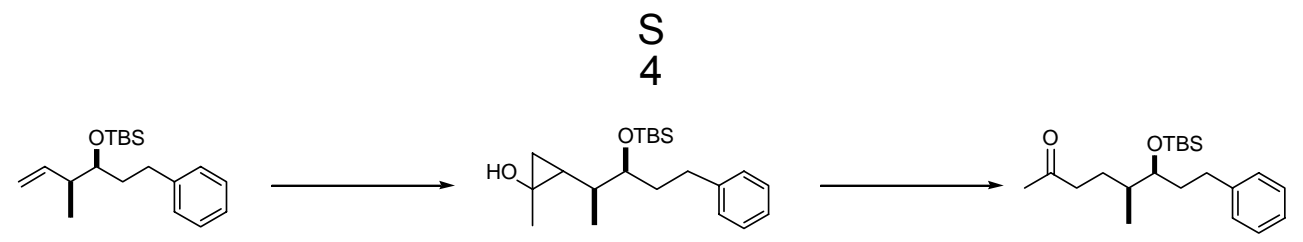

a) 2-[2-(tert-Butyl-dimethyl-silanyloxy)-1-methyl-4-phenyl-butyl]-1-methyl-cyclopropanol

${ }^{1} \mathrm{H}$ NMR (400 MHz, $\left.\mathrm{CDCl}_{3}\right) \delta$ 7.26- $7.30(\mathrm{~m}, 2 \mathrm{H}), 7.17-7.20(\mathrm{~m}, 3 \mathrm{H}), 3.73$ (dt, 1H, J=2.9, 6.7 Hz), 2.55$2.58(\mathrm{~m}, 2 \mathrm{H}), 1.80-1.84(\mathrm{~m}, 2 \mathrm{H}), 1.38(\mathrm{~s}, 3 \mathrm{H}), 1.09-1.16(\mathrm{~m}, 1 \mathrm{H}), 0.99-1.04(\mathrm{~m}, 1 \mathrm{H}), 0.95(\mathrm{~s}, 3 \mathrm{H}$, minor $\delta 0.93), 0.91(\mathrm{~s}, 9 \mathrm{H}), 0.82-0.85(\mathrm{~m}, 1 \mathrm{H}), 0.078-0.088(\mathrm{~m}, 7 \mathrm{H})$.

b) 6-(tert-Butyl-dimethyl-silanyloxy)-5-methyl-8-phenyl-octan-2-one

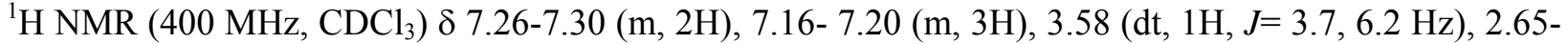
$2.73(\mathrm{~m}, 1 \mathrm{H}), 2.35-2.55(\mathrm{~m}, 3 \mathrm{H}), 2.14(\mathrm{~s}, 3 \mathrm{H}), 1.70-1.83(\mathrm{~m}, 4 \mathrm{H}), 1.25-1.40(\mathrm{~m}, 1 \mathrm{H}), 0.91(\mathrm{~s}, 9 \mathrm{H}), 0.85$ $(\mathrm{d}, 3 \mathrm{H}, J=6.78 \mathrm{~Hz}), 0.060(\mathrm{~s}, 3 \mathrm{H}), 0.032(\mathrm{~s}, 3 \mathrm{H}) ;{ }^{13} \mathrm{C} \mathrm{NMR}\left(400 \mathrm{MHz}, \mathrm{CDCl}_{3}\right) \delta 209.29,173.79,142.55$, $128.35,125.71,75.48,67.92,42.15,37.53,35.13,32.42,29.81,26.34,25.93,18.15,14.62,-4.26 ;[\alpha]_{D}$ 0.151 (c $0.62, \mathrm{CHCl}_{3}$ ) ; IR (thin film) 3026, 2955, 2856, 1719, 1603, 1471, 1359, 1254, 1164, 1061, 835, $773,699 \mathrm{~cm}^{-1}$; HRMS (EI) calculated for $\mathrm{C}_{21} \mathrm{H}_{36} \mathrm{O}_{2} \mathrm{SiNa}^{+}\left[\mathrm{M}+\mathrm{Na}^{+}\right]=371.237678$, found $371.237545 \pm$ $<1.0 \mathrm{ppm}$.

6-(tert-Butyl-dimethyl-silanyloxy)-5-methyl-8-phenyl-octan-2-one (Table 1, entry 5):

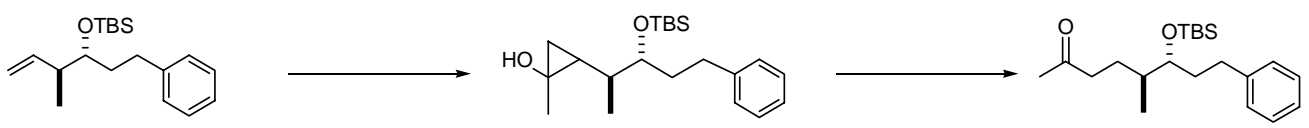

a) 2-[2-(tert-Butyl-dimethyl-silanyloxy)-1-methyl-4-phenyl-butyl]-1-methyl-cyclopropanol

$\mathrm{Ti}(\mathrm{i}-\mathrm{OPr})_{4}(0.14 \mathrm{~mL}$ of a $1.0 \mathrm{M}$ solution in hexanes, $0.49 \mathrm{mmol}, 3.0 \mathrm{eq})$ was added to a flame dried flask

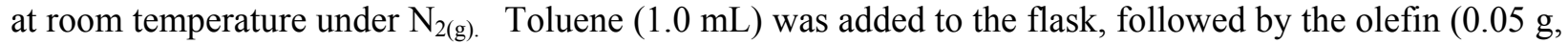
$0.16 \mathrm{mmol}, 1.0 \mathrm{eq})$ in toluene $(0.6 \mathrm{~mL})$ and EtOAc $(0.024 \mathrm{~mL}, 0.25 \mathrm{mmol}, 1.5 \mathrm{eq}$,). CyclohexylMgBr $\left(1.1 \mathrm{~mL}\right.$ of a $0.9 \mathrm{M}$ solution in $\left.\mathrm{Et}_{2} \mathrm{O}, 0.98 \mathrm{mmol}, 6 \mathrm{eq}\right)$ was then added at $25^{\circ} \mathrm{C}$ [NOTE when performing this reaction on a moderate to large scale $(>100 \mathrm{mg})$ the solution must be cooled to $0{ }^{\circ} \mathrm{C}$ before adding the cyclohexylMgBr. The reaction can be subsequently warmed to $25^{\circ} \mathrm{C}$ after addition is complete] and the reaction was stirred for $1 \mathrm{~h}$. $\operatorname{Ti}(i-\mathrm{OPr})_{4}(0.046 \mathrm{~mL}, 0.16 \mathrm{mmol}, 1.0 \mathrm{eq})$ and cyclohexylMgBr $(0.36 \mathrm{~mL}$ of a $0.9 \mathrm{M}$ solution in $\left.\mathrm{Et}_{2} \mathrm{O}, 0.33 \mathrm{mmol}, 2 \mathrm{eq}\right)$ were added again and the reaction was stirred for $1 \mathrm{~h}$ at 25 ${ }^{\circ} \mathrm{C}$, diluted with EtOAc $(50 \mathrm{~mL})$ and poured into $\mathrm{NH}_{4} \mathrm{Cl}(50 \mathrm{~mL})$. The mixture was stirred vigorously for $0.5 \mathrm{~h}$ to break up the emulsion and then filtered through celite. The layers were separated and the aqueous layer was extracted twice with EtOAc $\left(50 \mathrm{~mL}\right.$ x2). The organic layers were combined, dried with $\mathrm{Na}_{2} \mathrm{SO}_{4}$, filtered and concentrated under reduced pressure. The residue was purified by flash column chromatography $\left(20 \%\right.$ EtOAc /Hexanes). ${ }^{1} \mathrm{H}$ NMR $\left(400 \mathrm{MHz}, \mathrm{CDCl}_{3}\right) \delta$ 7.29- $7.35(\mathrm{~m}, 2 \mathrm{H}), 7.21-7.26$ $(\mathrm{m}, 3 \mathrm{H}), 3.71-3.74(\mathrm{~m}, 1 \mathrm{H}), 2.83-2.89(\mathrm{~m}, 1 \mathrm{H}), 2.54-2.60(\mathrm{~m}, 1 \mathrm{H}), 1.83-1.89(\mathrm{~m}, 1 \mathrm{H}), 1.73-1.80(\mathrm{~m}$, $1 \mathrm{H}), 1.39(\mathrm{~s}, 3 \mathrm{H}), 1.07-1.13(\mathrm{~m}, 1 \mathrm{H}), 0.95(\mathrm{~s}, 9 \mathrm{H}), 0.92-0.97(\mathrm{~m}, 3 \mathrm{H}), 0.84-0.90(\mathrm{~m}, 1 \mathrm{H}), 0.11(\mathrm{~s}, 3 \mathrm{H})$, $0.78-0.87(\mathrm{~m}, 1 \mathrm{H}), 0.049(\mathrm{~s}, 3 \mathrm{H})$.

b) 6-(tert-Butyl-dimethyl-silanyloxy)-5-methyl-8-phenyl-octan-2-one

${ }^{1} \mathrm{H}$ NMR $\left(400 \mathrm{MHz}, \mathrm{CDCl}_{3}\right) \delta$ 7.26- 7.35(m, 2H), 7.16-7.19 (m, 3H), 3.56 (q, 1H, J=4.8 Hz), 2.69- 2.77 $(\mathrm{m}, 1 \mathrm{H}), 2.45-2.57(\mathrm{~m}, 2 \mathrm{H}), 2.30-2.39(\mathrm{~m}, 1 \mathrm{H}), 2.13(\mathrm{~s}, 3 \mathrm{H}), 1.58-1.73(\mathrm{~m}, 4 \mathrm{H}), 1.29-1.38(\mathrm{~m}, 1 \mathrm{H})$, $0.91(\mathrm{~s}, 9 \mathrm{H}), 0.87(\mathrm{~d}, 3 \mathrm{H}, \mathrm{J}=6.8 \mathrm{~Hz}), 0.073(\mathrm{~s}, 3 \mathrm{H}), 0.041(\mathrm{~s}, 3 \mathrm{H}) ;{ }^{13} \mathrm{C}$ NMR $\left(400 \mathrm{MHz}, \mathrm{CDCl}_{3}\right) \delta 209.02$, 142.70, 128.32, 125. 64, 75.46, 41.81, 37.82, 34.42, 32.21, 29.81, 26.40, 25.90, 18.11, 14.31, -4.37, -4.39; $[\alpha]_{\mathrm{D}}+2.33\left(c \mathrm{1.3}, \mathrm{CHCl}_{3}\right)$; IR (thin film) 3026, 2955, 2856, 1719, 1603, 1471, 1359, 1255, 1165, 1061, 
835, 773, $699 \mathrm{~cm}^{-1}$; HRMS (EI) calculated for $\mathrm{C}_{21} \mathrm{H}_{36} \mathrm{O}_{2} \mathrm{SiNa}^{+}\left[\mathrm{M}+\mathrm{Na}^{+}\right]=371.237678$, found 371.237606 $\pm<1.0 \mathrm{ppm}$.

6-(tert-Butyl-dimethyl-silanyloxy)-6-phenyl-hexan-2-one (Table 1, entry 6):

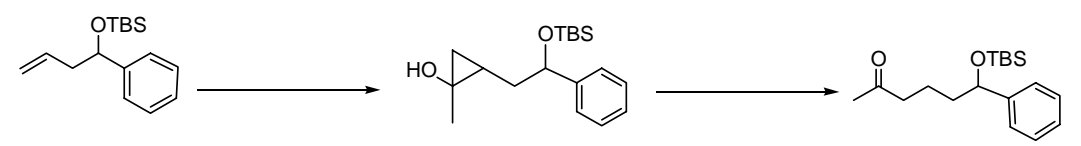

a) 2-[2-(tert-Butyl-dimethyl-silanyloxy)-2-phenyl-ethyl]-1-methyl-cyclopropanol

${ }^{1} \mathrm{H}$ NMR $\left(400 \mathrm{MHz}, \mathrm{CDCl}_{3}\right)$ 8 7.29- $7.32(\mathrm{~m}, 4 \mathrm{H}), 7.22$ - $7.25(\mathrm{~m}, 6 \mathrm{H}), 4.68-4.71(\mathrm{~m}, 2 \mathrm{H}), 1.82(\mathrm{p}, 1 \mathrm{H}$, $J=6.5 \mathrm{~Hz}), 1.71(\mathrm{~s}, 1 \mathrm{H}), 1.65(\mathrm{~s}, 1 \mathrm{H}), 1.61(\mathrm{dt}, 2 \mathrm{H}, J=1.9,7.0 \mathrm{~Hz}), 1.33-1.39(\mathrm{~m}, 1 \mathrm{H}), 1.23(\mathrm{~s}, 6 \mathrm{H}), 1.10-$ $1.18(\mathrm{~m}, 1 \mathrm{H}), 0.98-1.04(\mathrm{~m}, 1 \mathrm{H}), 0.89(\mathrm{~s}, 18 \mathrm{H}), 0.76-0.87(\mathrm{~m}, 2 \mathrm{H}), 0.017-0.085(\mathrm{~m}, 2 \mathrm{H}), 0.042(\mathrm{~s}, 6 \mathrm{H}),-$ $0.12(\mathrm{~s}, 6 \mathrm{H})$.

b) 6-(tert-Butyl-dimethyl-silanyloxy)-6-phenyl-hexan-2-one

${ }^{1} \mathrm{H}$ NMR $\left(400 \mathrm{MHz}, \mathrm{CDCl}_{3}\right) \delta$ 7.26- $7.30(\mathrm{~m}, 2 \mathrm{H}), 7.18-7.25(\mathrm{~m}, 3 \mathrm{H}), 4.64(\mathrm{t}, 1 \mathrm{H}, J=4.5 \mathrm{~Hz}), 2.39(\mathrm{t}, 2 \mathrm{H}$, $J=6.8 \mathrm{~Hz}), 2.09(\mathrm{~s}, 3 \mathrm{H}), 1.55-1.70(\mathrm{~m}, 4 \mathrm{H}), 0.89(\mathrm{~s}, 9 \mathrm{H}), 0.026(\mathrm{~s}, 3 \mathrm{H}),-0.15(\mathrm{~s}, 3 \mathrm{H}) ;{ }^{13} \mathrm{C}$ NMR $(400$ $\left.\mathrm{MHz}_{\mathrm{CDCl}}\right) \delta 208.87,145.29,127.99,126.86,125.76,74.72,43.56,40.18,29.74,25.80,19.92,18.16$, 4.69, -5.03; IR (thin film) 2954, 2856, 1718, 1361, 1256, 1095, 1061, 836, 775, $700 \mathrm{~cm}^{-1}$; HRMS (EI) calculated for $\mathrm{C}_{18} \mathrm{H}_{30} \mathrm{O}_{2} \mathrm{SiNa}^{+}\left[\mathrm{M}+\mathrm{Na}^{+}\right]=329.190728$, found $329.190357 \pm<1.1 \mathrm{ppm}$.

Decan-2-one (Table 1, entry 7):

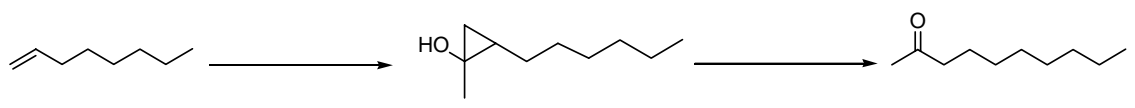

a) 2-Hexyl-1-methyl-cyclopropanol

${ }^{1} \mathrm{H}$ NMR (400 MHz, $\left.\mathrm{CDCl}_{3}\right) \delta 1.79(\mathrm{~s}, 1 \mathrm{H}), 1.40$ (s, 3H), 1.23-1.38 (m, 9H), 1.08-1.17 (m, 1H), 0.94$1.01(\mathrm{~m}, 1 \mathrm{H}), 0.88$ (t, 3H, $J=7.2 \mathrm{~Hz}), 0.84(\mathrm{~m}, 1 \mathrm{H}), 0.053$ (dd, $1 \mathrm{H}, J=5.2,1.1 \mathrm{~Hz})$.

b) Decan-2-one

${ }^{1} \mathrm{H}$ NMR (400 MHz, $\left.\mathrm{CDCl}_{3}\right) \delta 2.41(\mathrm{t}, 2 \mathrm{H}, J=7.5 \mathrm{~Hz}), 2.13(\mathrm{~s}, 3 \mathrm{H}), 1.55(\mathrm{p}, 3 \mathrm{H}, J=7.3 \mathrm{~Hz}), 1.21-1.31$ $(\mathrm{m}, 10 \mathrm{H}), 0.87(\mathrm{t}, 3 \mathrm{H}, J=6.8 \mathrm{~Hz}) ;{ }^{13} \mathrm{C}$ NMR $\left(400 \mathrm{MHz}, \mathrm{CDCl}_{3}\right) \delta 209.45,43.81,31.79,29.85,29.34$, 29.16, 29.12, 23.84, 22.62, 14.08; IR (thin film) 2926, 1718, 1460, 1357, 1162, $715 \mathrm{~cm}^{-1}$; HRMS (EI) calculated for $\mathrm{C}_{10} \mathrm{H}_{20} \mathrm{ONa}^{+}\left[\mathrm{M}+\mathrm{Na}^{+}\right]=179.140636$, found $179.140555 \pm<1.0 \mathrm{ppm}$.

7-(tert-Butyl-dimethyl-silanyloxy)-2-methyl-7-phenyl-heptan-3-one (Table 1, entry 8):

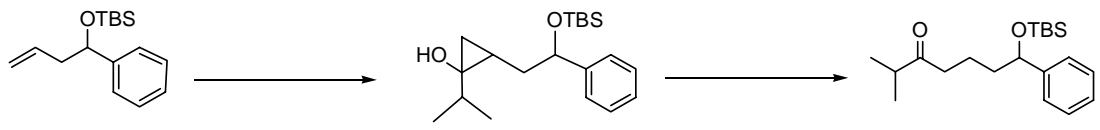

a) 2-[2-(tert-Butyl-dimethyl-silanyloxy)-2-phenyl-ethyl]-1-isopropyl-cyclopropanol 
$\mathrm{Ti}(\mathrm{i}-\mathrm{OPr})_{4}(0.33 \mathrm{~mL}$ of a $1.0 \mathrm{M}$ solution in hexanes, $1.14 \mathrm{mmol}, 3.0 \mathrm{eq})$ was added to a flame dried flask

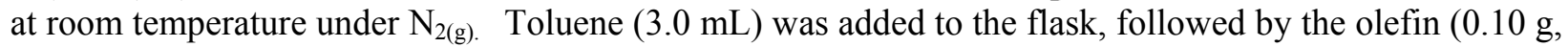
$0.38 \mathrm{mmol}, 1.0 \mathrm{eq})$ in toluene $(0.8 \mathrm{~mL})$ and methylisobutyrate $(0.066 \mathrm{~mL}, 0.57 \mathrm{mmol}, 1.5 \mathrm{eq}$,). CyclohexylMgBr $\left(2.28 \mathrm{~mL}\right.$ of a $1.0 \mathrm{M}$ solution in $\left.\mathrm{Et}_{2} \mathrm{O}, 2.28 \mathrm{mmol}, 6 \mathrm{eq}\right)$ was then added at $0{ }^{\circ} \mathrm{C}$ and the reaction was stirred for $1 \mathrm{~h}$. Ti(i-OPr $)_{4}(0.11 \mathrm{~mL}, 0.38 \mathrm{mmol}, 1.0 \mathrm{eq})$ and cyclohexylMgBr $(0.76 \mathrm{~mL}$ of a $1.0 \mathrm{M}$ solution in $\left.\mathrm{Et}_{2} \mathrm{O}, 0.76 \mathrm{mmol}, 2 \mathrm{eq}\right)$ were added again at $0{ }^{\circ} \mathrm{C}$ and the reaction was stirred for $1 \mathrm{~h}$ at $25^{\circ} \mathrm{C}$, diluted with EtOAc $(50 \mathrm{~mL})$ and poured into $\mathrm{NH}_{4} \mathrm{Cl}(50 \mathrm{~mL})$. The mixture was stirred vigorously for $0.5 \mathrm{~h}$ to break up the emulsion and then filtered through celite. The layers were separated and the aqueous layer was extracted twice with EtOAc $(50 \mathrm{~mL}$ x2). The organic layers were combined, dried with $\mathrm{Na}_{2} \mathrm{SO}_{4}$, filtered and concentrated under reduced pressure. The residue was purified by flash column chromatography (20\% EtOAc /Hexanes). (1:1 mixture of diastereoisomers) ${ }^{1} \mathrm{H}$ NMR $\left(400 \mathrm{MHz}, \mathrm{CDCl}_{3}\right) \delta$ 7.21-7.32 (m, 10H), $4.69(\mathrm{t}, 1 \mathrm{H}, J=6.4 \mathrm{~Hz}), 4.63(\mathrm{dd}, 1 \mathrm{H}, J=4.2,8.7 \mathrm{~Hz}), 2.12-2.17(\mathrm{~m}, 1 \mathrm{H}), 1.97-2.02$ $(\mathrm{m}, 1 \mathrm{H}), 1.23-1.39(\mathrm{~m}, 4 \mathrm{H}), 1.05-1.11(\mathrm{~m}, 8 \mathrm{H}), 1.02(\mathrm{~d}, 3 \mathrm{H}, J=6.8 \mathrm{~Hz}), 0.99(\mathrm{~d}, 3 \mathrm{H}, J=7.0 \mathrm{~Hz}), 0.79-$ $0.93(\mathrm{~m}, 6 \mathrm{H}), 0.88(\mathrm{~s}, 9 \mathrm{H}), 0.87(\mathrm{~s}, 9 \mathrm{H}), 0.083(\mathrm{dd}, 2 \mathrm{H}, J=5.5,6.5 \mathrm{~Hz}), 0.037(\mathrm{~s}, 3 \mathrm{H}), 0.028(\mathrm{~s}, 3 \mathrm{H}),-$ $0.14(\mathrm{~s}, 3 \mathrm{H}),-0.15(\mathrm{~s}, 3 \mathrm{H})$.

b) 7-(tert-Butyl-dimethyl-silanyloxy)-2-methyl-7-phenyl-heptan-3-one

${ }^{1} \mathrm{H}$ NMR $\left(400 \mathrm{MHz}, \mathrm{CDCl}_{3}\right) \delta$ 7.17- $7.30(\mathrm{~m}, 5 \mathrm{H}), 4.61-4.64(\mathrm{~m}, 1 \mathrm{H}), 2.53(\mathrm{sp}, 1 \mathrm{H}, J=6.9 \mathrm{~Hz}), 2.40(\mathrm{t}$, $2 \mathrm{H}, J=7.0 \mathrm{~Hz}), 1.47-1.71(\mathrm{~m}, 4 \mathrm{H}), 1.04(\mathrm{~d}, 3 \mathrm{H}, J=2.9 \mathrm{~Hz}), 1.03(\mathrm{~d}, 3 \mathrm{H}, \mathrm{J}=3.0 \mathrm{~Hz}) 0.86(\mathrm{~s}, 9 \mathrm{H})$, $0.0019(\mathrm{~s}, 3 \mathrm{H}),-0.18(\mathrm{~s}, 3 \mathrm{H}) ;{ }^{13} \mathrm{C}$ NMR $\left(400 \mathrm{MHz}, \mathrm{CDCl}_{3}\right) \delta 214.68,145.47,128.00,126.86,125.80$, 74.91, 40.67, 40.41, 40.21, 25.83, 19.96, 18.23, -4.65, -4.98; IR (thin film) 2956, 2930, 2857, 1712, 1467, 1363, 1254, 1092, 1068, $1006 \mathrm{~cm}^{-1}$; HRMS (EI) calculated for $\mathrm{C}_{20} \mathrm{H}_{34} \mathrm{O}_{2} \mathrm{SiNa}^{+}\left[\mathrm{M}+\mathrm{Na}^{+}\right]=357.2220$, found $357.2226 \pm<1.6 \mathrm{ppm}$.

\section{2-[4,6-Bis-(tert-butyl-dimethyl-silanyloxy)-hexyl]-1-(3-hydroxy-butyl)-cyclopropanol (5)}

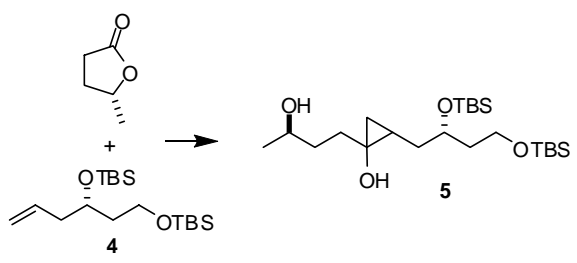

$\mathrm{Ti}(\mathrm{i}-\mathrm{OPr})_{4}(0.435 \mathrm{~mL}$ of a $1.0 \mathrm{M}$ solution in hexanes, $0.435 \mathrm{mmol}, 3.0 \mathrm{eq})$ was added to a flame dried flask at room temperature under $\mathrm{N}_{2(\mathrm{~g})}$. Toluene $(1.0 \mathrm{~mL}$ ) was added to the flask, followed by olefin (4) $(0.05 \mathrm{~g}, 0.15 \mathrm{mmol}, 1.0 \mathrm{eq})$ in toluene $(0.5 \mathrm{~mL})$ and $(\mathrm{R})-\gamma$-valerolactone $(0.22 \mathrm{~mL}$ of a $1 \mathrm{M}$ THF solution, $0.22 \mathrm{mmol}, 1.5 \mathrm{eq}$,). CyclohexylMgBr $\left(1.09 \mathrm{~mL}\right.$ of a $0.88 \mathrm{M}$ solution in $\left.\mathrm{Et}_{2} \mathrm{O}, 0.87 \mathrm{mmol}, 6 \mathrm{eq}\right)$ was then added at $25{ }^{\circ} \mathrm{C}$ [NOTE when performing this reaction on a moderate to large scale $(>100 \mathrm{mg})$ the solution must be cooled to $0{ }^{\circ} \mathrm{C}$ before adding the cyclohexylMgBr. The reaction can be subsequently warmed to $25^{\circ} \mathrm{C}$ after addition is complete] and the reaction was stirred for $1 \mathrm{~h}$. Ti(i-OPr $)_{4}(0.13 \mathrm{~mL}$ $, 0.44 \mathrm{mmol}, 3.0 \mathrm{eq})$ and cyclohexylMgBr $\left(1.09 \mathrm{~mL}\right.$ of a $0.88 \mathrm{M}$ solution in $\mathrm{Et}_{2} \mathrm{O}, 0.87 \mathrm{mmol}$, 6 eq) were added again and the reaction was stirred for $1 \mathrm{~h}$ at $25^{\circ} \mathrm{C}$, diluted with EtOAc $(50 \mathrm{~mL})$ and poured into $\mathrm{NH}_{4} \mathrm{Cl}(50 \mathrm{~mL})$. The mixture was stirred vigorously for $0.5 \mathrm{~h}$ to break up the emulsion and then filtered through celite. The layers were separated and the aqueous layer was extracted twice with EtOAc $(50 \mathrm{~mL}$ x2). The organic layers were combined, dried with $\mathrm{Na}_{2} \mathrm{SO}_{4}$, filtered and concentrated under reduced pressure. The residue was purified by flash column chromatography ( $20 \%$ EtOAc /Hexanes) to provide 5 $(0.060 \mathrm{~g}, 92 \%)$ as an oil $\left(3: 1\right.$ mixture of diastereoisomers). ${ }^{1} \mathrm{H}$ NMR $\left(500 \mathrm{MHz}, \mathrm{CDCl}_{3}\right) \delta 3.82-3.96(\mathrm{~m}$, $2 \mathrm{H}), 3.67(\mathrm{t}, 2 \mathrm{H}, J=6.6 \mathrm{~Hz}), 1.59-1.80(\mathrm{~m}, 8 \mathrm{H}), 1.24(\mathrm{~d}, 3 \mathrm{H}, J=6.2 \mathrm{~Hz})$, minor 1.25, 1.04- $1.14(\mathrm{~m}$, $2 \mathrm{H}), 0.88(\mathrm{~s}, 18 \mathrm{H}), 0.094-0.14(\mathrm{~m}, 1 \mathrm{H}), 0.052(\mathrm{mH}, \mathrm{s}), 0.041(\mathrm{~m}, 6 \mathrm{H}) ;{ }^{13} \mathrm{C} \mathrm{NMR}\left(400 \mathrm{MHz}, \mathrm{CDCl}_{3}\right)$ 
$\delta$ minor $69.46,69.42$, minor $68.39,68.21,59.91$, minor $58.48,58.05$, minor $40.10,39.90$, minor 37.52 , 37.35 , minor $35.86,35.73,31.07,25.93$, 25.89, minor $23.90,23.71$, minor $22.70,22.06,19.94$, 18.24, 18.05, minor $-4.32,-4.40$, minor $-4.64,-4.67,-5.27$; $[\alpha]_{\mathrm{D}}+12.32$ (c 1.0, $\mathrm{CHCl}_{3}$ ); IR (thin film) 3326, 2954, 2930, 2857, 1469, 1254, 1095 (thin film): $\mathrm{cm}^{-1}$; HRMS (EI): calculated for $\mathrm{C}_{23} \mathrm{H}_{51} \mathrm{O}_{4} \mathrm{Si}_{2}\left[\mathrm{M}+\mathrm{H}^{+}\right]=$ 447.3320 , found $447.3311 \pm 2.1 \mathrm{ppm}$.

\section{9, 11-Bis-(tert-butyl-dimethyl-silanyloxy)-2-hydroxy-undecan-5-one (6)}

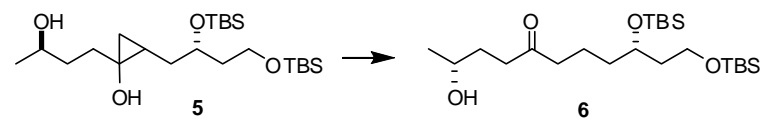

$\mathrm{Bu}_{3} \mathrm{SnH}(0.071 \mathrm{~mL}, 0.13 \mathrm{mmol}, 2$ eq. $)$ was added to the cyclopropanol (5) (0.060g, $\left.0.13 \mathrm{mmol}\right)$ in DMF $(0.37 \mathrm{~mL})$ at $0{ }^{\circ} \mathrm{C}$ in a flame dried flask under $\mathrm{N}_{2(\mathrm{~g})}$. $\mathrm{Fe}\left(\mathrm{NO}_{3}\right)_{3}(0.30 \mathrm{~mL}, 1 \mathrm{M}$ solution in DMF, $2.2 \mathrm{eq})$ was then added over $30 \mathrm{~min}$. (via syringe pump) to the cooled solution. The reaction was stirred for another $30 \mathrm{~min}$ at $0{ }^{\circ} \mathrm{C}$ and then warmed to room temperature and stirred for $1 \mathrm{~h}$. Upon completion the reaction was quenched with $\mathrm{H}_{2} \mathrm{O}(50 \mathrm{~mL})$. The aqueous layer was extracted twice with EtOAc $(50 \mathrm{~mL} \times 4)$ and the organic layers were combined, washed with saturated aqueous $\mathrm{NaCl}(50 \mathrm{~mL})$, dried with $\mathrm{MgSO}_{4}$, filtered and concentrated under reduced pressure. The residue was purified by flash column chromatography (20\% EtOAc /Hexanes) to provide $6(0.045 \mathrm{~g}, 75 \%)$ as an oil. ${ }^{1} \mathrm{H}$ NMR $\left(400 \mathrm{MHz}, \mathrm{CDCl}_{3}\right) \delta 3.74-3.83$ $(\mathrm{m}, 2 \mathrm{H}), 3.64(\mathrm{t}, 2 \mathrm{H}, J=6.6 \mathrm{~Hz}), 2.54(\mathrm{t}, 2 \mathrm{H}, \mathrm{J}=7.1 \mathrm{~Hz}), 2.42(\mathrm{t}, 2 \mathrm{H}, \mathrm{J}=7.3 \mathrm{~Hz}), 1.70-1.80(\mathrm{~m}, 2 \mathrm{H})$, 1.58- $1.67(\mathrm{~m}, 4 \mathrm{H}), 1.39-1.47(\mathrm{~m}, 2 \mathrm{H}), 1.19(\mathrm{~d}, 3 \mathrm{H}, \mathrm{J}=6.2), 0.874(\mathrm{~s}, 9 \mathrm{H}), 0.870(\mathrm{~s}, 9 \mathrm{H}), 0.027(\mathrm{~s}, 12 \mathrm{H})$ $;{ }^{13} \mathrm{C}$ NMR $\left(400 \mathrm{MHz}, \mathrm{CDCl}_{3}\right) \delta 211.66,68.96,67.51,59.82,43.02,39.93,38.98,36.77,32.56,25.87$, $23.71,19.51,18.24,18.06,-4.45,-4.61,-5.33 ;[\alpha]_{\mathrm{D}}+16.75\left(c 0.75, \mathrm{CHCl}_{3}\right)$; IR (thin film) 3416, 2954, 2930, 2857, 1713, 1463, 1376, 1254, 1094, 1005, $938 \mathrm{~cm}^{-1}$; HRMS (EI) calculated for $\mathrm{C}_{23} \mathrm{H}_{50} \mathrm{O}_{4} \mathrm{Si}_{2}$ $\left[\mathrm{M}+\mathrm{Na}^{+}\right]=469.3139$, found $469.3149 \pm 2 \mathrm{ppm}$.

\section{2-(2-Methyl-1,6-dioxa-spiro[4.5]dec-7-yl)-ethanol (7)}

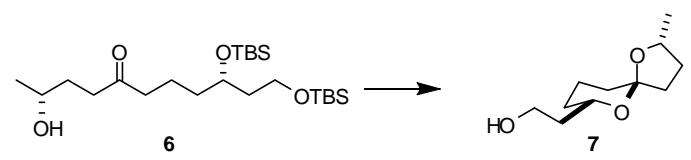

Aqueous HF (0.36 mL, $2.49 \mathrm{mmol}, 2.8 \mathrm{eq})$ was added to ketone (6) $(0.40 \mathrm{~g}, 0.90 \mathrm{mmol}, 1 \mathrm{eq})$ in acetonitrile $(8.95 \mathrm{~mL})$ at $25^{\circ} \mathrm{C}$ in a Nalgene bottle under $\mathrm{N}_{2(\mathrm{~g})}$. The solution was stirred for $16 \mathrm{~h}$ and was then quenched with $\mathrm{Na}_{2} \mathrm{CO}_{3}(50 \mathrm{~mL})$. The aqueous layer was extracted with EtOAc $(50 \mathrm{~mL} \times 2)$ and the organic layers were combined, washed with saturated aqueous $\mathrm{NaCl}(50 \mathrm{~mL})$, dried with $\mathrm{MgSO}_{4}$, filtered and concentrated under reduced pressure. The residue was purified by flash column chromatography (15\% EtOAc, $\left.1 \% \mathrm{Et}_{3} \mathrm{~N} / \mathrm{Hexanes}\right)$ to provide $7(0.160 \mathrm{~g}, 89 \%)$ as an oil.

${ }^{1} \mathrm{H}$ NMR $\left(500 \mathrm{MHz}, \mathrm{CDCl}_{3}\right) \delta 4.20$ (sextet, $\left.1 \mathrm{H}, J=6.6 \mathrm{~Hz}\right), 4.03(\mathrm{tq}, 1 \mathrm{H}, J=2.5,9.1 \mathrm{~Hz}), 3.74-3.80(2 \mathrm{H}$, $\mathrm{m}), 2.07-2.14(1 \mathrm{H}, \mathrm{m}), 1.63-1.90(7 \mathrm{H}, \mathrm{m}), 1.52-1.55(1 \mathrm{H}, \mathrm{M}), 1.41-1.48(1 \mathrm{H}, \mathrm{m}), 1.31-1.34(2 \mathrm{H}, \mathrm{m}), 1.25$ $(\mathrm{d}, 3 \mathrm{H}, J=6.2 \mathrm{~Hz}) ;{ }^{13} \mathrm{C} \mathrm{NMR}\left(400 \mathrm{MHz}, \mathrm{CDCl}_{3}\right) \delta 106.21,74.01,71.87,62.30,37.96,37.56,33.18$, $31.12,30.83,21.25,20.03 ;[\alpha]_{\mathrm{D}}+69.24\left(c 0.50, \mathrm{CHCl}_{3}\right)$; IR (thin film) 3426, 2939, 2872, 1440, 1383, 1221, 1160, 1068, 1029, 945, $877 \mathrm{~cm}^{-1}$; HRMS (EI) calculated for $\mathrm{C}_{11} \mathrm{H}_{20} \mathrm{O}_{3}\left[\mathrm{M}+\mathrm{Na}^{+}\right]=223.1304$, found $223.1295 \pm 4.3 \mathrm{ppm}$.

\section{7-(2-Bromo-ethyl)-2-methyl-1,6-dioxa-spiro[4.5]decane (8)}




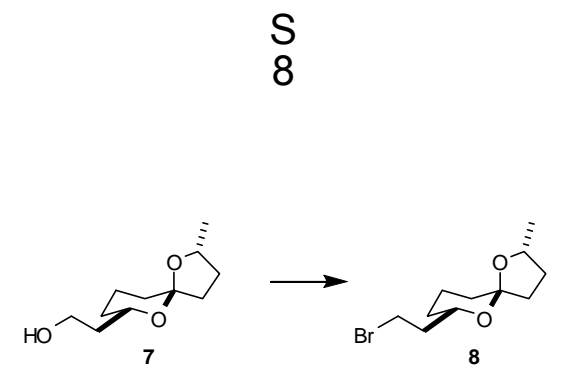

$\mathrm{PPh}_{3}(0.051 \mathrm{~g}, 0.19 \mathrm{mmol}, 1.1 \mathrm{eq})$ and $\mathrm{N}$-bromosuccinimide $(0.034 \mathrm{~g}, 0.19 \mathrm{mmol}, 1.1 \mathrm{eq})$ were added to a solution of alcohol (7) $(0.035 \mathrm{~g}, 0.18 \mathrm{mmol}, 1 \mathrm{eq})$ in $\mathrm{CH}_{2} \mathrm{Cl}_{2}(1.8 \mathrm{~mL})$ at $0{ }^{\circ} \mathrm{C}$ in a flame dried flask under $\mathrm{N}_{2(\mathrm{~g})}$. The reaction was stirred at $0{ }^{\circ} \mathrm{C}$ for $30 \mathrm{~min}$ and then warmed to $\mathrm{rt}$ for $1 \mathrm{~h}$. The solvent was removed under reduced pressure and the resulting material was passed through a plug of silica gel with a $1 \%$ EtOAc/ $1 \% \mathrm{Et}_{3} \mathrm{~N} /$ hexanes solution to provide $8(0.045 \mathrm{~g}, 99 \%)$ as a yellow oil. ${ }^{1} \mathrm{H}$ NMR $(500 \mathrm{MHz}$, $\left.\mathrm{CDCl}_{3}\right) \delta 4.23$ (sextet, $\left.1 \mathrm{H}, J=6.3 \mathrm{~Hz}\right), 3.93-3.98(\mathrm{~m}, 1 \mathrm{H}), 3.45-3.53(2 \mathrm{H}, \mathrm{m}), 2.09-2.16(1 \mathrm{H}, \mathrm{m}), 1.83-$ $1.98(4 \mathrm{H}, \mathrm{m}), 1.72-1.78(1 \mathrm{H}, \mathrm{M}), 1.64-1.69(2 \mathrm{H}, \mathrm{m}), 1.53-1.57(1 \mathrm{H}, \mathrm{m}), 1.38-1.45(\mathrm{~m}, 2 \mathrm{H}), 1.24(\mathrm{~d}, 3 \mathrm{H}, J$ $=6.3 \mathrm{~Hz}), 1.18-1.26(\mathrm{~m}, 1 \mathrm{H}) ;{ }^{13} \mathrm{C} \mathrm{NMR}\left(400 \mathrm{MHz}, \mathrm{CDCl}_{3}\right) \delta 105.97,73.84,67.69,39.15,37.82,33.25$, $31.24,30.63,30.30,21.26,20.25 ;[\alpha]_{\mathrm{D}}+62.28\left(c 0.10, \mathrm{CHCl}_{3}\right)$; IR (thin film) 2940, 2871, 1440, 1384, $1264,1220,1075,980,928,880 \mathrm{~cm}^{-1}$; HRMS (EI) calculated for $\mathrm{C}_{11} \mathrm{H}_{20} \mathrm{O}_{2} \mathrm{Br}\left[\mathrm{M}+\mathrm{H}^{+}\right]=263.0641$, found $263.0641 \pm 0.5 \mathrm{ppm}$.

Diethyl-carbamic acid 1-(2-bromo-3,5-dimethoxy-phenyl)-but-3-enyl ester (10)

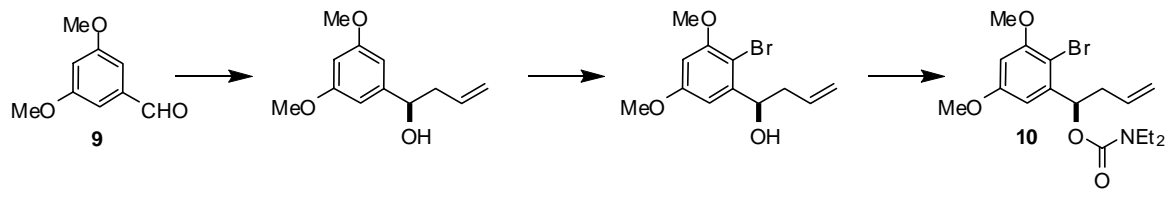

a) 1-(3,5-Dimethoxy-phenyl)-but-3-en-1-ol

Allyl magnesium bromide $\left(28.89 \mathrm{~mL}, 28.89 \mathrm{mmol}, 1.2 \mathrm{eq}, 1 \mathrm{M}\right.$ solution in $\left.\mathrm{Et}_{2} \mathrm{O}\right)$ was added to a solution of (+)-IPC ${ }_{2} \mathrm{BOMe}(9.149,28.89 \mathrm{mmol}, 1.2 \mathrm{eq})$ and $\mathrm{Et}_{2} \mathrm{O}(60.2 \mathrm{~mL})$ at $-78^{\circ} \mathrm{C}$ in a flame dried flask under $\mathrm{N}_{2(\mathrm{~g})}$. The resulting cloudy, white solution was warmed to $\mathrm{rt}$ for $1 \mathrm{~h}$ and then returned to $-78^{\circ} \mathrm{C}$, at which point the aldehyde $(4.0 \mathrm{~g}, 24.07 \mathrm{mmol}, 1.0 \mathrm{eq})$ was added dropwise in $\mathrm{Et}_{2} \mathrm{O}(5 \mathrm{~mL})$. The reaction was stirred for $2 \mathrm{~h}$ at $-78{ }^{\circ} \mathrm{C}$ and then warmed to $\mathrm{rt}$ for $1 \mathrm{~h}$. Upon completion, the flask was cooled to $0{ }^{\circ} \mathrm{C}$ and $\mathrm{NaOH}(21.4 \mathrm{~mL})$ and $\mathrm{H}_{2} \mathrm{O}_{2}(11.6 \mathrm{~mL})$ were added dropwise via addition funnel. To complete to oxidation process, the reaction was refluxed for $45 \mathrm{~min}$. The layers were separated and the organic layer was dried with $\mathrm{MgSO}_{4}$, filtered and concentrated under reduced pressure. The residue was purified by flash column chromatography (5\% EtOAc, /Hexanes) to provide $(3.75 \mathrm{~g}, 75 \%)$ as an oil. ${ }^{1} \mathrm{H}$ NMR $(500$ $\left.\mathrm{MHz}, \mathrm{CDCl}_{3}\right) \delta 6.53(\mathrm{~s}, 1 \mathrm{H}), 6.52(\mathrm{~s}, 1 \mathrm{H}), 6.38(\mathrm{t}, 1 \mathrm{H}, J=2.3 \mathrm{~Hz}), 5.85-5.94(\mathrm{~m}, 1 \mathrm{H}), 5.17(\mathrm{dd}, 1 \mathrm{H}, J=$ $1.8,15.8 \mathrm{~Hz}), 5.15(\mathrm{dd}, 1 \mathrm{H}, J=1.9,9.1 \mathrm{~Hz}), 4.67(\mathrm{dd}, 1 \mathrm{H}, \mathrm{J}=4.9,7.8 \mathrm{~Hz}), 3.80(\mathrm{~s}, 6 \mathrm{H}), 2.44-2.55(\mathrm{~m}$, $2 \mathrm{H}) ;{ }^{13} \mathrm{C} \mathrm{NMR}\left(400 \mathrm{MHz}, \mathrm{CDCl}_{3}\right) \delta 160.78,146.48,134.38,118.38,103.64,99.36,73.27,55.29$, 43.68; $[\alpha]_{\mathrm{D}}+31.70\left(c 1.1, \mathrm{CHCl}_{3}\right)$; IR (thin film) 3426, 3000, 2937, 2838, 1598, 1463, 1429, 1349, 1295 , 1204, 1155, 1059, $920 \mathrm{~cm}^{-1}$; HRMS (EI) calculated for $\mathrm{C}_{12} \mathrm{H}_{17} \mathrm{O}_{3}\left[\mathrm{M}+\mathrm{H}^{+}\right]=209.1172$, found $209.1173 \pm$ $0.4 \mathrm{ppm}$.

b) 1-(2-Bromo-3,5-dimethoxy-phenyl)-but-3-en-1-ol

NBS (2.81 g, $15.80 \mathrm{mmol}, 1 \mathrm{eq})$ was added to the alcohol in $\mathrm{CHCl}_{3}(80 \mathrm{~mL})$ in a flame dried flask under $\mathrm{N}_{2(\mathrm{~g})}$. The solution was refluxed for $1.5 \mathrm{~h}$, and then concentrated under reduced pressure. The residue was purified by flash column chromatography (10\% EtOAc, /Hexanes) to provide $(3.63 \mathrm{~g}, 80 \%)$ as an oil. ${ }^{1} \mathrm{H}$ NMR $\left(500 \mathrm{MHz}, \mathrm{CDCl}_{3}\right) \delta 6.77(\mathrm{~d}, 1 \mathrm{H}, J=2.9 \mathrm{~Hz}), 6.41(\mathrm{~d}, 1 \mathrm{H}, J=2.7 \mathrm{~Hz}), 5.85-5.94(\mathrm{~m}, 1 \mathrm{H})$, 
$5.20(\mathrm{dd}, 1 \mathrm{H}, J=1.4,17.0 \mathrm{~Hz}), 5.18(\mathrm{dd}, 1 \mathrm{H}, J=0.55,9.2 \mathrm{~Hz}), 5.14(\mathrm{ddd}, 1 \mathrm{H}, \mathrm{J}=3.4,6.9,8.6 \mathrm{~Hz}), 3.87$ $(\mathrm{s}, 3 \mathrm{H}), 3.82(\mathrm{~s}, 3 \mathrm{H}), 2.61-2.66(\mathrm{~m}, 1 \mathrm{H}), 2.31(\mathrm{dt}, 1 \mathrm{H}, J=8.4,16.4 \mathrm{~Hz}), 2.23(\mathrm{~d}, 1 \mathrm{H}, J=3.3 \mathrm{~Hz}) ;{ }^{13} \mathrm{C}$ NMR (400 MHz, $\left.\mathrm{CDCl}_{3}\right) \delta 159.93,156.30,144.85,134.41,118.56,102.97,101.92,98.81,71.94,56.29$, $55.55,41.90 ;[\alpha]_{\mathrm{D}}+51.04\left(c 1.15, \mathrm{CHCl}_{3}\right)$; IR (thin film) 3419, 3003, 2938, 1588, 1454, 1430, 1323, $1284,1200,1160,1052,1022,919 \mathrm{~cm}^{-1}$; HRMS (EI) calculated for $\mathrm{C}_{12} \mathrm{H}_{16} \mathrm{O}_{3} \mathrm{Br}\left[\mathrm{M}+\mathrm{H}^{+}\right]=287.0277$, found $287.0287 \pm 3.4 \mathrm{ppm}$.

c) Diethyl-carbamic acid 1-(2-bromo-3,5-dimethoxy-phenyl)-but-3-enyl ester (10)

$\mathrm{NaH}(0.33 \mathrm{~g}, 13.67 \mathrm{mmol}, 1.2 \mathrm{eq}$, washed with dry hexanes) was added to stirred solution of the alcohol (3.27 g, $11.39 \mathrm{mmol}, 1 \mathrm{eq})$ in THF $(115 \mathrm{~mL})$ at $0{ }^{\circ} \mathrm{C}$ in a flame dried flask under $\mathrm{N}_{2(\mathrm{~g})}$. After $45 \mathrm{~min}$ diethylcarbamyl chloride $(1.73 \mathrm{~mL}, 13.67 \mathrm{mmol}, 1.2 \mathrm{eq})$ was added and the reaction was warmed to $\mathrm{rt}$ and stirred for $16 \mathrm{~h}$. The mixture was then quenched with $\mathrm{Na}_{2} \mathrm{CO}_{3}(100 \mathrm{~mL})$. The aqueous layer was extracted twice with EtOAc $(100 \mathrm{~mL} \times 2)$ and the organic layers were combined, washed with saturated aqueous $\mathrm{NaCl}(100 \mathrm{~mL})$, dried with $\mathrm{MgSO}_{4}$, filtered and concentrated under reduced pressure. The residue was purified by flash column chromatography (10\% EtOAc /Hexanes) to provide $(3.42 \mathrm{~g}, 82 \%)$ as an oil. ${ }^{1} \mathrm{H}$ NMR $\left(400 \mathrm{MHz}, \mathrm{CDCl}_{3}\right) \delta 6.52(\mathrm{~d}, 1 \mathrm{H}, J=2.8 \mathrm{~Hz}), 6.39(\mathrm{~d}, 1 \mathrm{H}, J=2.8), 6.09$ (q, $1 \mathrm{H}, J=4.2$ $\mathrm{Hz}), 5.76-5.86(\mathrm{~m}, 1 \mathrm{H}), 5.08(\mathrm{dd}, 1 \mathrm{H}, J=1.9,15.8 \mathrm{~Hz}), 5.05(\mathrm{dd}, 1 \mathrm{H}, J=1.9,9.0 \mathrm{~Hz}), 3.84(\mathrm{~s}, 3 \mathrm{H}), 3.78$ $(\mathrm{s}, 6 \mathrm{H}), 3.28-3.33(\mathrm{~m}, 4 \mathrm{H}), 2.59-2.66(\mathrm{~m}, 1 \mathrm{H}), 2.46-2.54(\mathrm{~m}, 1 \mathrm{H}), 1.09-1.19(\mathrm{~m}, 6 \mathrm{H}){ }^{13} \mathrm{C}$ NMR $(400$ $\left.\mathrm{MHz}, \mathrm{CDCl}_{3}\right) \delta 159.67,156.48,154.72,142.63,133.55,117.65,103.38,102.12,98.31,74.79,56.23$, 55.30, 41.83, 41.21, 39.59, $14.25,13.39 ;[\alpha]_{\mathrm{D}}-10.03\left(c \mathrm{1.2}, \mathrm{CHCl}_{3}\right)$; IR (thin film) 2974, 2836, 1702, $1588,1422,1325,1276,1164,1068 \mathrm{~cm}^{-1}$; HRMS (EI) calculated for $\mathrm{C}_{17} \mathrm{H}_{25} \mathrm{O}_{4} \mathrm{BrN}\left[\mathrm{M}+\mathrm{H}^{+}\right]=386.0961$, found $386.0956 \pm 1.4 \mathrm{ppm}$.

3-Allyl-5,7-dimethoxy-3H-isobenzofuran-1-one (11)

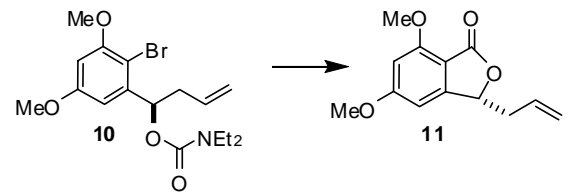

$t$-BuLi $(1.04 \mathrm{~mL}$, in a $1.7 \mathrm{M}$ pentane solution, $1.76 \mathrm{mmol}, 2.0 \mathrm{eq})$ was added quickly to carbamate (10) $(0.34 \mathrm{~g}, 0.88 \mathrm{mmol}, 1.0 \mathrm{eq})$ in THF $(8.8 \mathrm{~mL})$ at $-78^{\circ} \mathrm{C}$ in a flame dried flask under $\mathrm{N}_{2(\mathrm{~g})}$. The reaction was kept at $-78{ }^{\circ} \mathrm{C}$ for $1.5 \mathrm{~h}$ and then allowed to warm to $\mathrm{rt}$ for $1 \mathrm{~h}$ and $45 \mathrm{~min}$, at which time the reaction was quenched with $p$-toluenesulfonic acid $(0.67 \mathrm{~g}, 3.52 \mathrm{mmol}, 4 \mathrm{eq})$ and stirred vigorously for $16 \mathrm{~h}$. The cloudy solution was poured into $\mathrm{Na}_{2} \mathrm{CO}_{3}(50 \mathrm{~mL})$. The aqueous layer was extracted twice with EtOAc (50 $\mathrm{mL} \mathrm{x} 2)$ and the organic layers were combined, washed with saturated aqueous $\mathrm{NaCl}(50 \mathrm{~mL})$, dried with $\mathrm{MgSO}_{4}$, filtered and concentrated under reduced pressure. The residue was purified by flash column chromatography (50\% EtOAc /Hexanes) to provide $11(0.12 \mathrm{~g}, 79 \%)$ as solid. ${ }^{1} \mathrm{H}$ NMR (400 MHz, $\left.\mathrm{CDCl}_{3}\right) \delta 6.44(\mathrm{~s}, 1 \mathrm{H}), 6.41(\mathrm{~s}, 1 \mathrm{H}), 5.70-5.80(\mathrm{~m}, 1 \mathrm{H}), 5.33(\mathrm{t}, 1 \mathrm{H}, J=5.9 \mathrm{~Hz}) 5.17(\mathrm{dd}, 1 \mathrm{H}, J=1.5$, $17.1 \mathrm{~Hz}), 5.12(\mathrm{dd}, 1 \mathrm{H}, J=1.2,11.5 \mathrm{~Hz}), 3.93(\mathrm{~s}, 3 \mathrm{H}), 3.88(\mathrm{~s}, 3 \mathrm{H}), 2.73-2.66(\mathrm{~m}, 1 \mathrm{H}), 2.52-2.60(\mathrm{~m}$, 1H) ${ }^{13} \mathrm{C} \mathrm{NMR}\left(400 \mathrm{MHz}, \mathrm{CDCl}_{3}\right) \delta 168.21,166.61,159.55,154.30,131.35,119.41,106.91,98.69,97.64$, 78.81, 55.95, 55.89, 38.71; $[\alpha]_{\mathrm{D}}+67.55\left(\mathrm{c} 1.2, \mathrm{CHCl}_{3}\right)$; IR (thin film) 3079, 3009, 2941, 2842, 1747, 1604, 1493, 1463., 1429, 1222, 1059, 998, 934, $902 \mathrm{~cm}^{-1}$; HRMS (EI) calculated for $\mathrm{C}_{13} \mathrm{H}_{15} \mathrm{O}_{4}\left[\mathrm{M}+\mathrm{H}^{+}\right]=$ 235.0964 , found $235.0968 \pm 1.3 \mathrm{ppm}$. 


\section{(+)-Spirolaxine methyl ether}

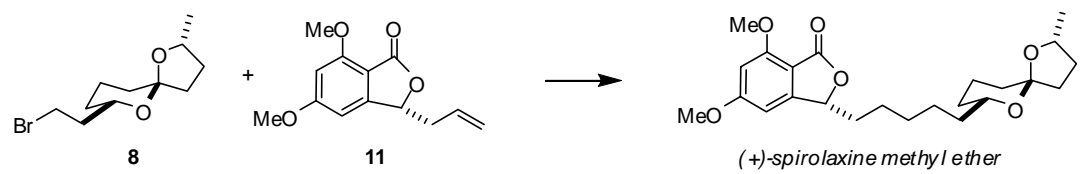

9-BBN $(0.58 \mathrm{~mL}$ of $0.5 \mathrm{M}$ THF solution, $0.29 \mathrm{mmol}, 1.7 \mathrm{eq})$ was added to olefin 11 (0.045 g, $0.26 \mathrm{mmol}$, $1.5 \mathrm{eq})$ at $25^{\circ} \mathrm{C}$ in a flame dried flask under $\mathrm{Ar}_{(\mathrm{g})}$. The solution was stirred for $4 \mathrm{~h}$ at $\mathrm{rt}$, then $\mathrm{Cs}_{2} \mathrm{CO}_{3}($ $0.043 \mathrm{~mL}$ of a $10 \mathrm{M}$ solution in $\mathrm{H}_{2} \mathrm{O}, 0.43 \mathrm{mmol}, 2.5 \mathrm{eq}$ ) was added and the stirring was continued for 10 min. The resulting solution was cannulated into a flask containing alkyl bromide $8(0.045 \mathrm{~g}, 0.17 \mathrm{mmol}$, $1.0 \mathrm{eq}), \mathrm{Pd}(\mathrm{OAc})_{2}(0.0019 \mathrm{~g}, 0.0086 \mathrm{mmol})$ and $\mathrm{PCy}_{3}(0.0048 \mathrm{~g}, 0.017 \mathrm{mmol})$ under $\mathrm{Ar}_{(\mathrm{g})}$. The flask and cannula were rinsed once with dioxane $(0.30 \mathrm{~mL}$, the solvent was degassed by performing a freeze, pump, thaw cycle 3 times prior to use). The reaction mixture was stirred at $40{ }^{\circ} \mathrm{C}$ for $20 \mathrm{~h}$, then diluted with EtOAc $(5 \mathrm{~mL})$ and flushed through a plug of celite. The residue was concentrated under reduced pressure and purified by flash column chromatography $\left(30 \% \mathrm{EtOAc} / 1 \% \mathrm{Et}_{3} \mathrm{~N} / \mathrm{Hexanes}\right)$ to provide spirolaxine methyl ether $(0.56 \mathrm{mg}, 79 \%)$ as an oil. ${ }^{1} \mathrm{H}$ NMR $\left(400 \mathrm{MHz}, \mathrm{CDCl}_{3}\right) \delta 6.40(\mathrm{~s}, 1 \mathrm{H}), 6.39(\mathrm{~s}, 1 \mathrm{H}), 5.28$ (dd, $1 \mathrm{H}, J=3.8,7.7 \mathrm{~Hz}$ ), 4.12 (sextet, $1 \mathrm{H}, J=6.5 \mathrm{~Hz}$ ), 3.93 (s, 3H), $3.88(\mathrm{~s}, 3 \mathrm{H}) 3.64-3.69$ (m, 1H), 2.06$2.15(1 \mathrm{H}, \mathrm{m}), 1.92-1.99(1 \mathrm{H}, \mathrm{m}), 1.59-1.88(7 \mathrm{H}, \mathrm{M}), 1.50-1.54(1 \mathrm{H}, \mathrm{m}), 1.30-1.42(9 \mathrm{H}, \mathrm{m}), 1.21$ (d, 3H, J $=6.2 \mathrm{~Hz}), 1.14(\mathrm{dddd}, 1 \mathrm{H}, \mathrm{J}=3.9,13.1,13.1,16.7 \mathrm{~Hz}) ;{ }^{13} \mathrm{C}$ NMR $\left(400 \mathrm{MHz}, \mathrm{CDCl}_{3}\right) \delta 168.51,166.60$, 159.52 , 155.11, 106.86, 105.96, 98.53, 97.32, 79.87, 73.58, 69.87, 55.93, 55.86, 37.93, 36.06, 34.72, $33.44,31.29,30.90,29.30,25.40,24.50,21.23,20.35 ;[\alpha]_{\mathrm{D}}+62.9\left(\right.$ c $\left.0.22, \mathrm{CHCl}_{3}\right)\left[\mathrm{Lit}^{1}:+62(c\right.$ 0.22, $\left.\left.\mathrm{CHCl}_{3}\right)\right]$; IR (thin film) 2934, 2861, 1755, 1610, 1493, 1459, 1433, 1338, 1219, 1158, 1053, 1031, 981 $\mathrm{cm}^{-1}$; HRMS (EI) calculated for $\mathrm{C}_{24} \mathrm{H}_{35} \mathrm{O}_{6}\left[\mathrm{M}+\mathrm{H}^{+}\right]=419.2428$, found $419.2442 \pm 3.3 \mathrm{ppm}$. 


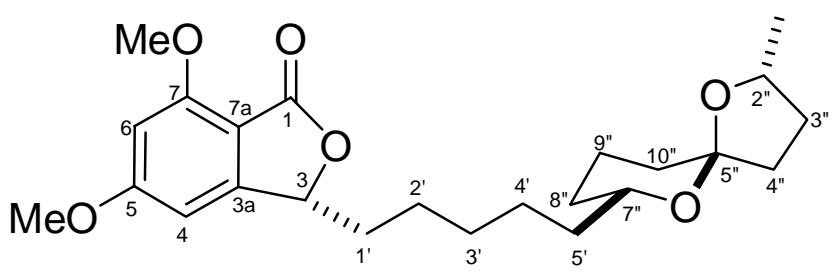

Comparison of data for synthetic and natural $(+)$-spirolaxine methyl ether

\begin{tabular}{|c|c|c|c|c|}
\hline & Literature $^{2}$ & & Synthetic & \\
\hline & ${ }^{1} \mathrm{H} \delta($ mult,$J)$ & ${ }^{13} \mathrm{C} \delta$ & ${ }^{1} \mathrm{H} \delta($ mult,$J)$ & ${ }^{13} \mathrm{C} \delta^{3}$ \\
\hline 1 & - & 168.5 & - & 168.5 \\
\hline 3 & $5.30(\mathrm{dd}, 7.8,3.8)$ & 79.9 & $5.28(\mathrm{dd}, 3.8,7.7)$ & 79.9 \\
\hline $3 a$ & - & 155.2 & - & 155.1 \\
\hline 4 & $6.42(\mathrm{~d}, 1.7)$ & 98.6 & $6.40(\mathrm{~s})$ & 98.5 \\
\hline 5 & - & 166.6 & - & 166.6 \\
\hline 6 & $6.40(\mathrm{~s})$ & 97.3 & $6.39(\mathrm{~s})$ & 97.3 \\
\hline 7 & - & 159.6 & - & 159.5 \\
\hline $7 \mathrm{a}$ & - & 107.0 & - & 106.9 \\
\hline 1 ' & $\begin{array}{l}1.59-1.72(\mathrm{~m})_{\mathrm{a}}, 1.94-2.01 \\
(\mathrm{~m})_{\mathrm{b}}\end{array}$ & 34.8 & $1.59-1.88(\mathrm{~m})_{\mathrm{a}}, 1.92-1.99(\mathrm{~m})_{\mathrm{b}}$ & 34.7 \\
\hline $2^{\prime}$ & N.R. & 24.5 & $1.30-1.42(\mathrm{~m})$ & 24.5 \\
\hline $3^{\prime}$ & $1.25-1.48(\mathrm{~m})$ & 29.3 & $1.30-1.42(\mathrm{~m})$ & 29.3 \\
\hline 4 & $1.25-1.48(\mathrm{~m})$ & 25.4 & $1.30-1.42(\mathrm{~m})$ & 25.4 \\
\hline 5 & $1.25-1.48(\mathrm{~m})$ & 36.1 & $1.30-1.42(\mathrm{~m})$ & 36.1 \\
\hline $\mathrm{Me}$ & $1.23(\mathrm{~d}, 6.6)$ & 21.3 & $1.21(\mathrm{~d}, 6.2)$ & 21.2 \\
\hline $2 "$ & $4.14(\mathrm{dq}, 6.6,6.6)$ & 73.9 & $4.12(\mathrm{tq}, 6.2,6.8)$ & 73.6 \\
\hline $3 ”$ & $\begin{array}{l}1.25-1.48 \quad(\mathrm{~m})_{\mathrm{a}}, \\
(\mathrm{dddd}, 11.9,8.8,6.6,6.6)_{\mathrm{b}}\end{array}$ & 31.3 & $1.30-1.42(\mathrm{~m})_{\mathrm{a}}, \quad 2.06-2.15(\mathrm{~m})_{\mathrm{b}}$ & 31.3 \\
\hline $4 "$ & $\begin{array}{l}1.74 \quad(\mathrm{ddd}, \quad 12.7, \quad 10.4, \\
6.6)_{\mathrm{a}}, 1.80-1.89(\mathrm{~m})_{\mathrm{b}}\end{array}$ & 38.0 & $1.59-1.88(\mathrm{~m})$ & 38.0 \\
\hline $5 "$ & - & 106.0 & - & 106.0 \\
\hline $7 "$ & $3.66-3.72(\mathrm{~m})$ & 69.9 & $3.64-3.69(\mathrm{~m})$ & 69.9 \\
\hline $8 "$ & $\begin{array}{l}1.14 \text { (dddd, 13.0, 13.0, } \\
13.0,3.8)_{\mathrm{a}}, 1.51-1.56(\mathrm{~m})_{\mathrm{b}}\end{array}$ & 30.9 & $\begin{array}{l}1.14(\mathrm{dddd}, 3.9,13.1,13.1,16.7)_{\mathrm{a}}, \\
1.50-1.54(\mathrm{~m})_{\mathrm{b}}\end{array}$ & 30.9 \\
\hline 9" & $\begin{array}{l}1.59-1.72 \quad(\mathrm{~m})_{\mathrm{a}}, \quad 1.80-1.89 \\
(\mathrm{~m})_{\mathrm{b}}\end{array}$ & 20.4 & $1.59-1.88(\mathrm{~m})$ & 20.4 \\
\hline $10 "$ & $1.59-1.72(\mathrm{~m})$ & 33.5 & $1.59-1.88(\mathrm{~m})$ & 33.4 \\
\hline $\mathrm{MeO}$ & $3.89(\mathrm{~s})$ & 55.9 & $3.88(\mathrm{~s})$ & 55.9 \\
\hline $\mathrm{MeO}$ & $3.95(\mathrm{~s})$ & 56.0 & $3.93(\mathrm{~s})$ & 56.0 \\
\hline
\end{tabular}

*not reported

${ }^{1}$ T. Adachi, I. Takagi, K. Kondo, A. Kawashima, A. Kobayashi, I. Taneoka, S. Morimoto, B. M. Hi and Z. Chen, PCT Int. Appl., 1996, W0 9610020.

${ }^{2}$ M. A. Gaudliana, L. H. Huang, T. Kaneko and P. C. Watts, PCT Int. Appl., 1996 , W0 9605204. 


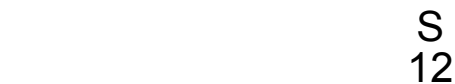

$\mathrm{S}$
12

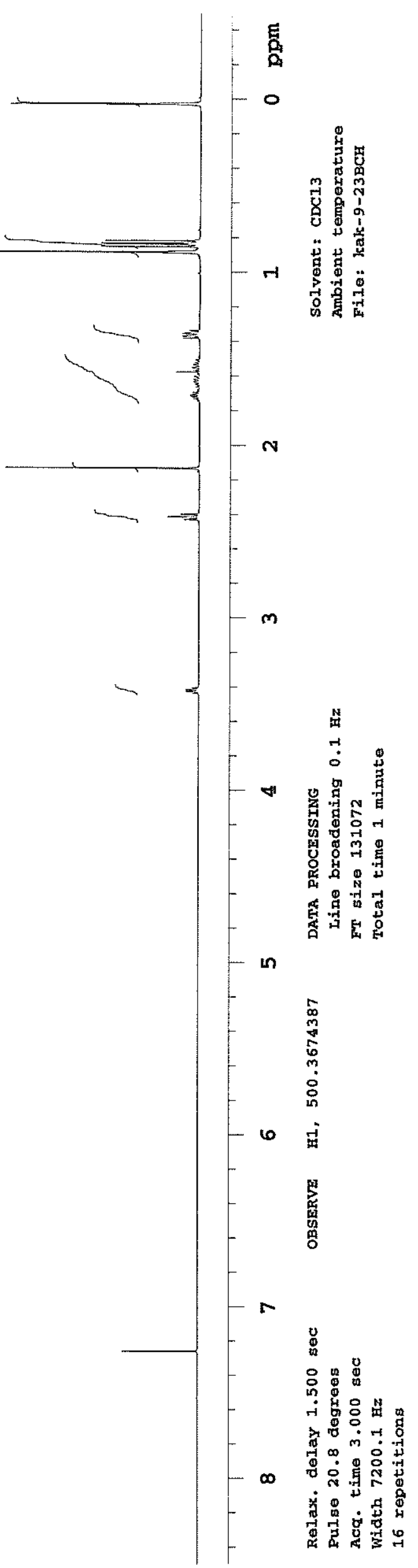




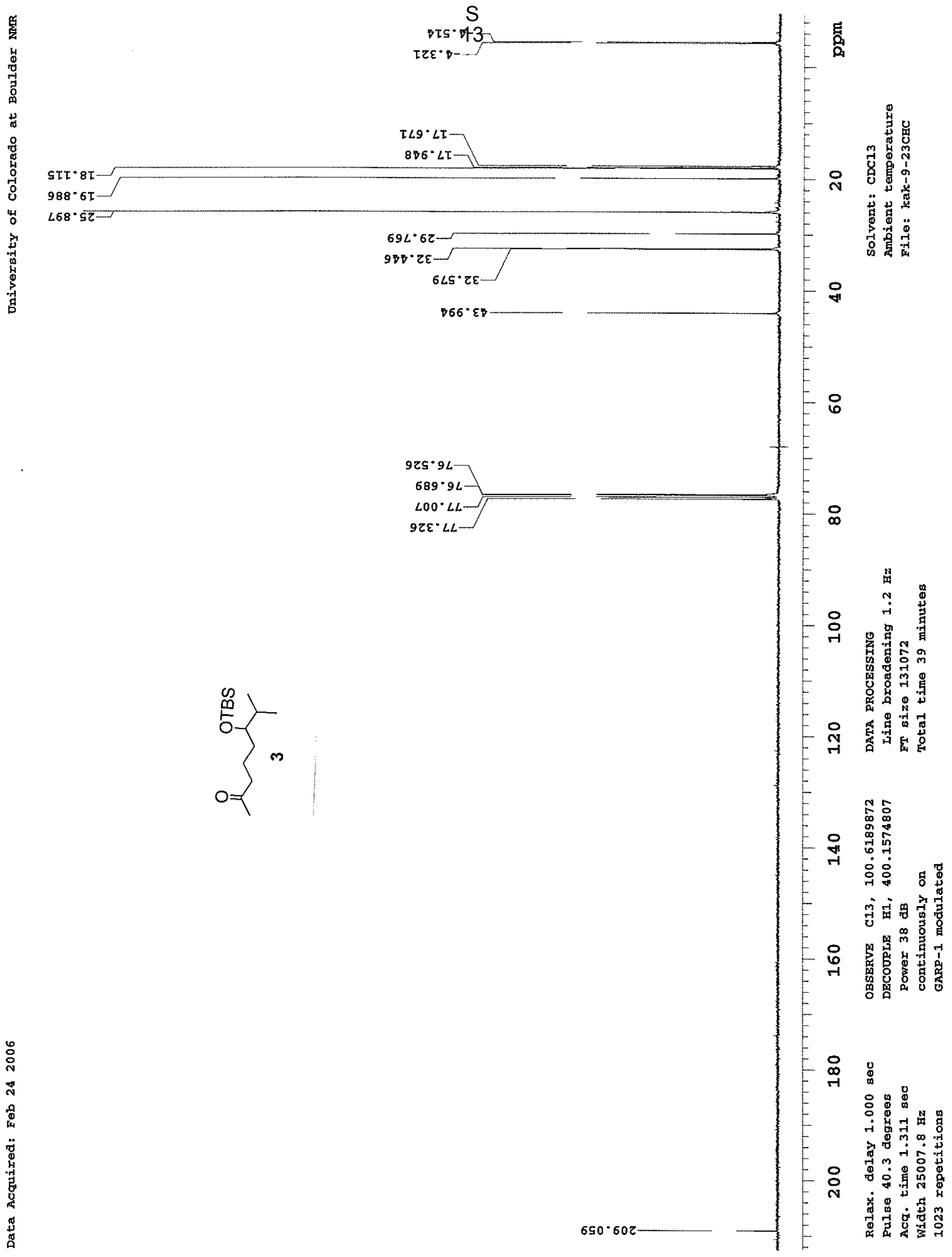




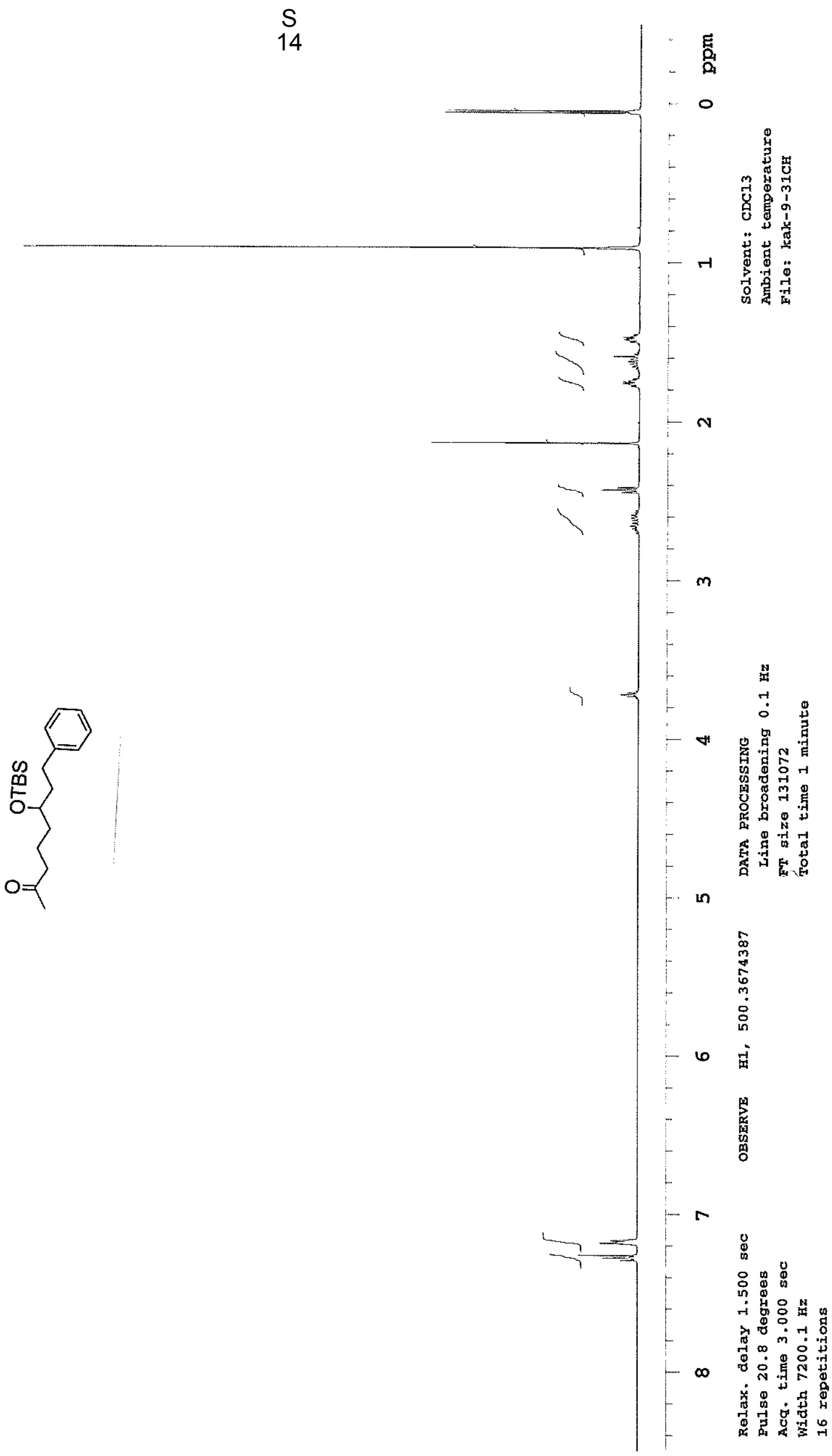




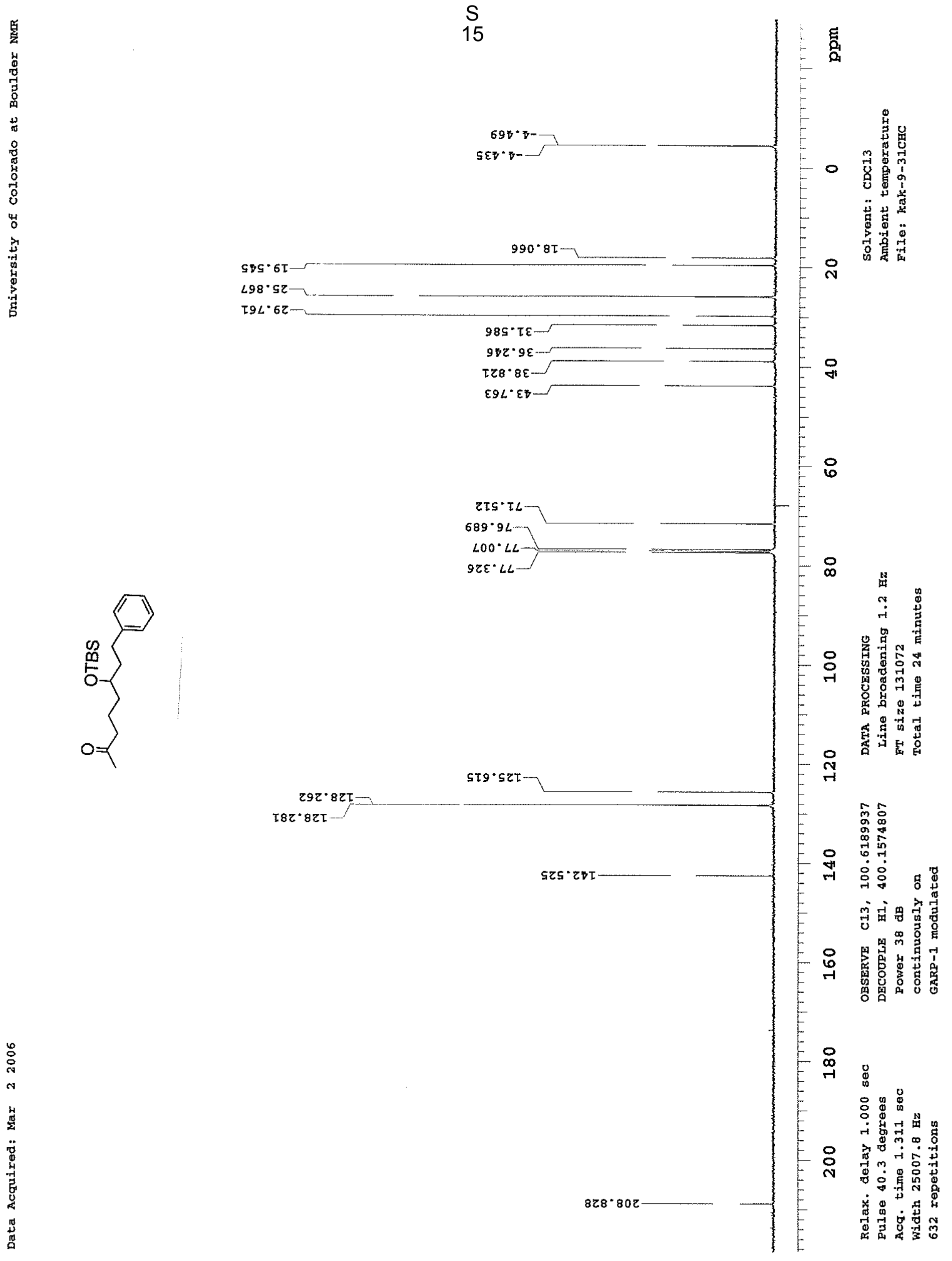




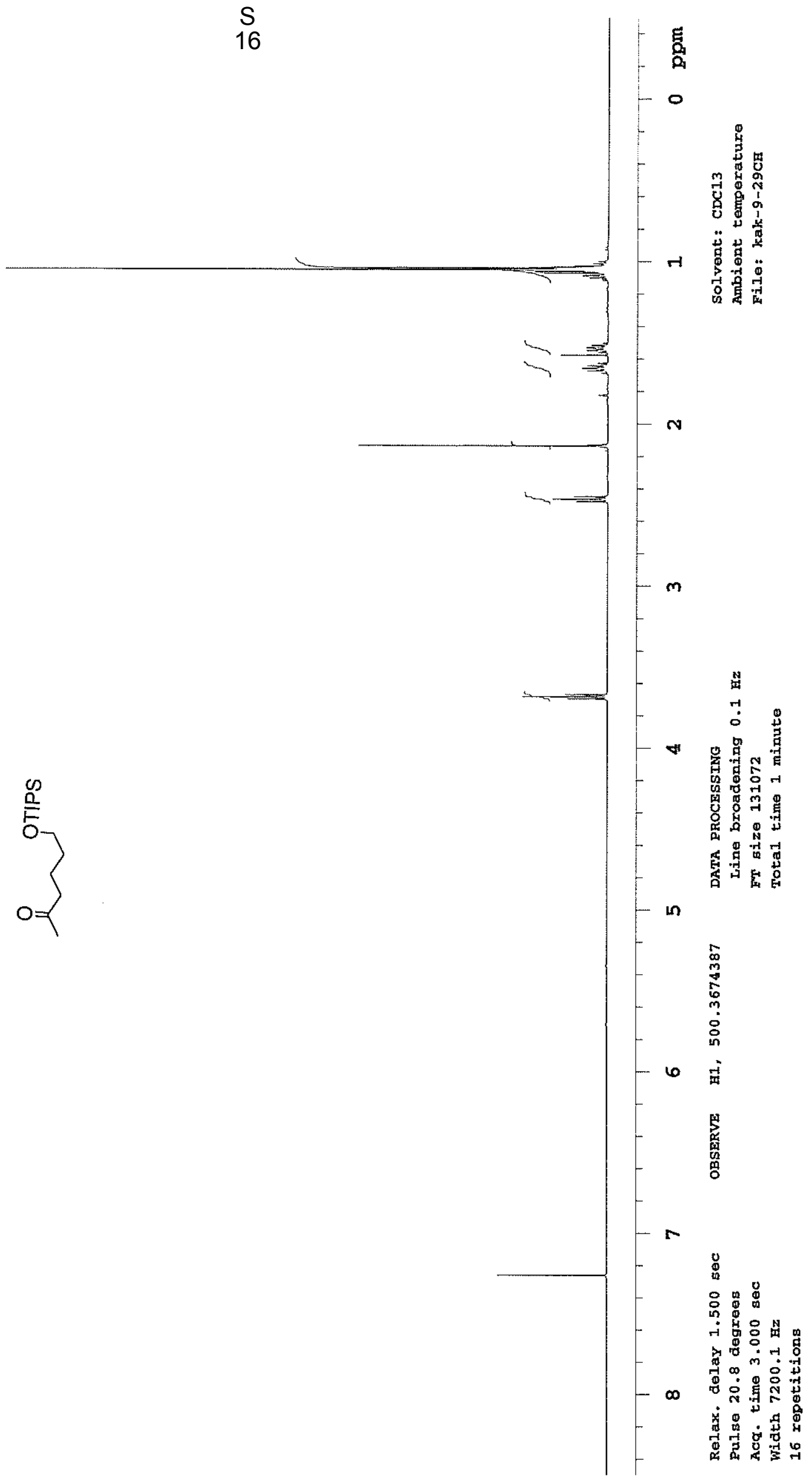




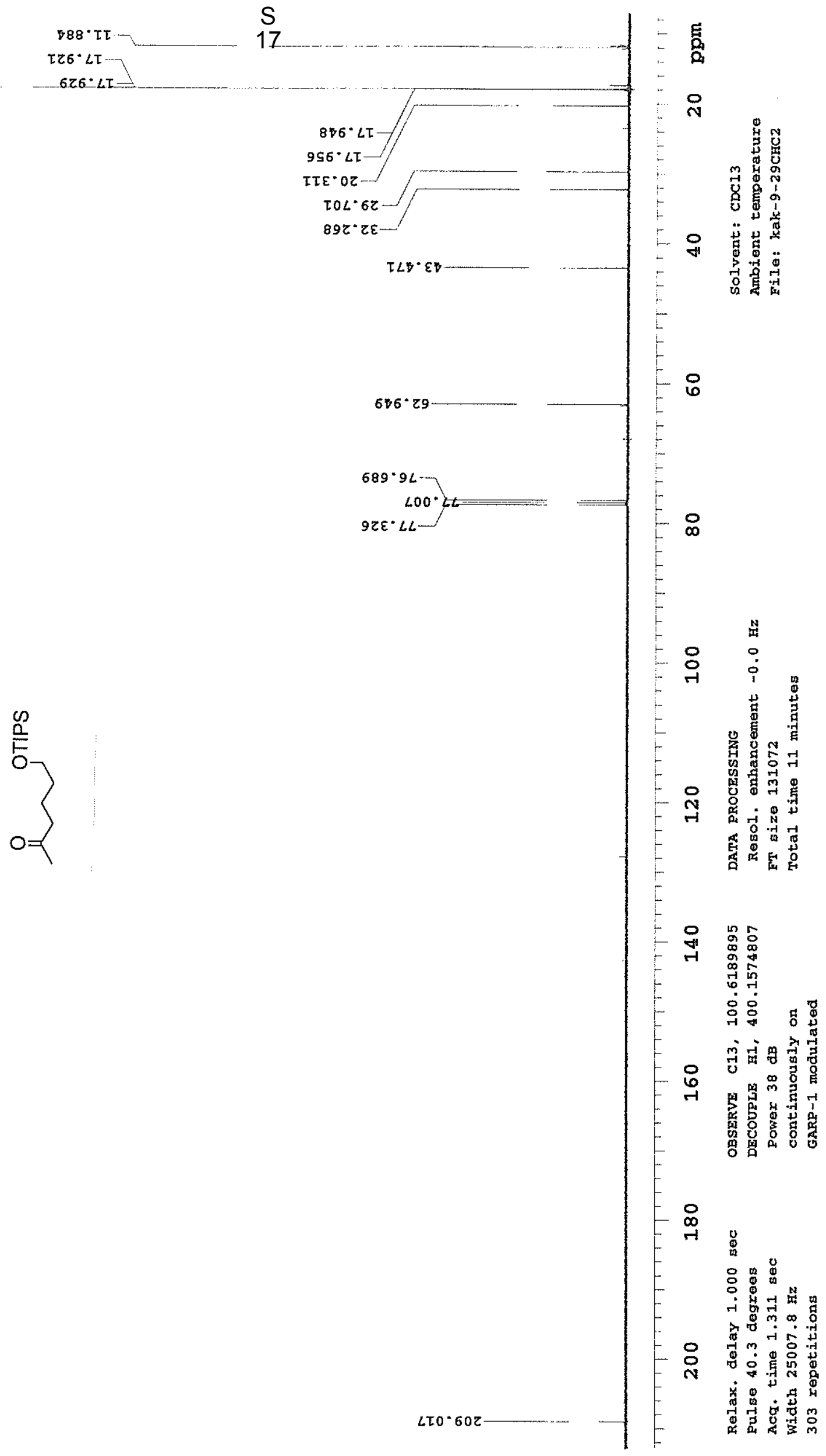




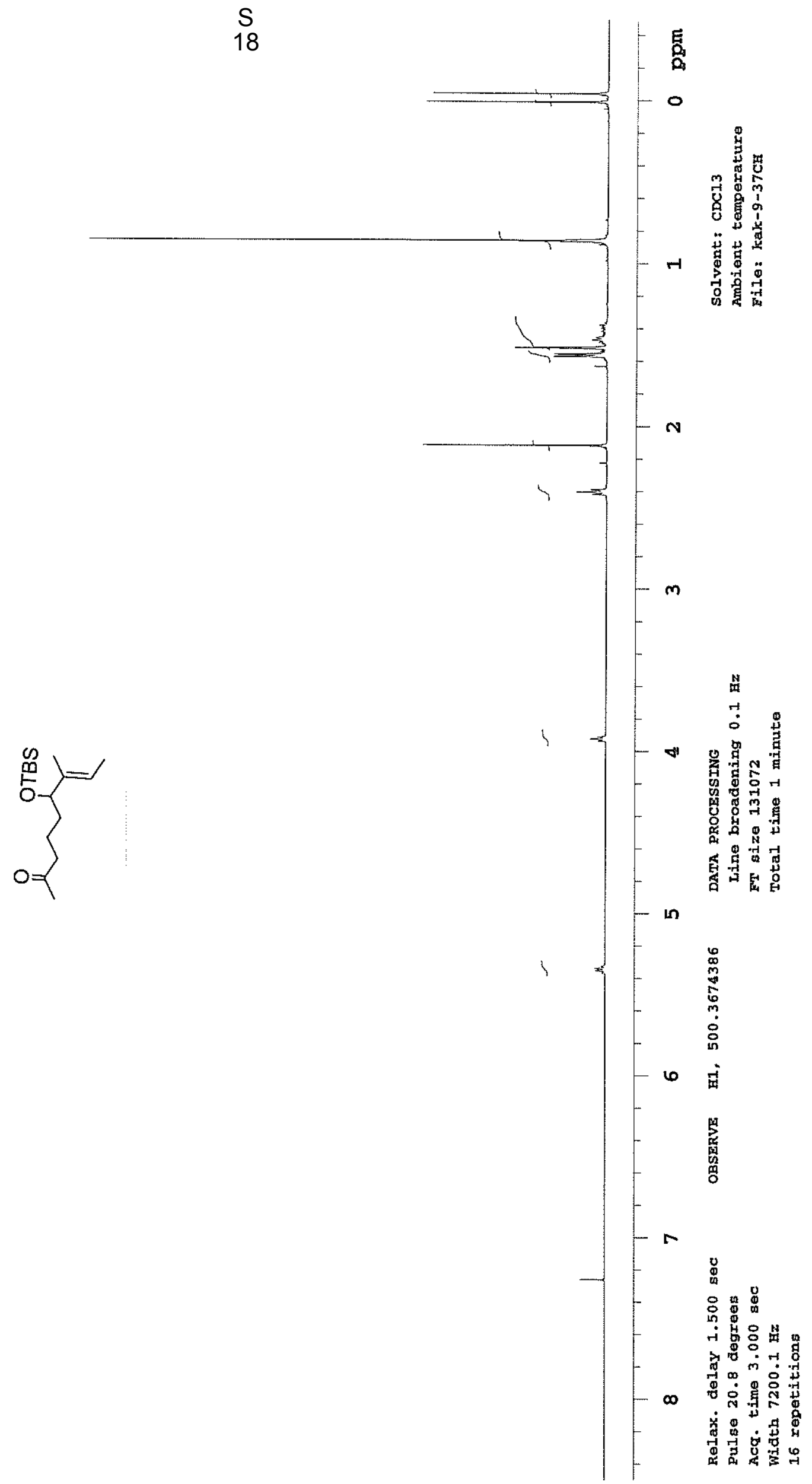




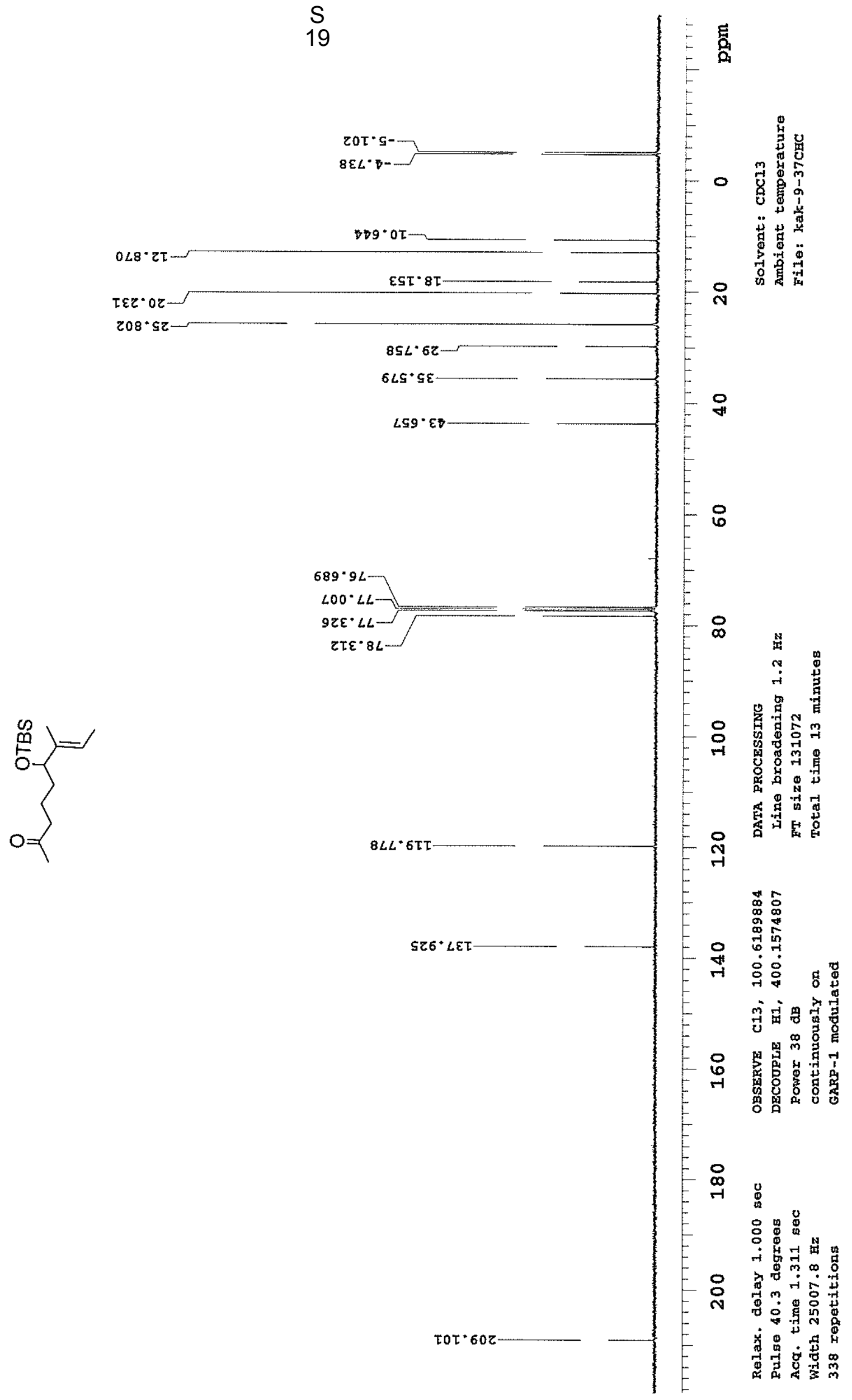




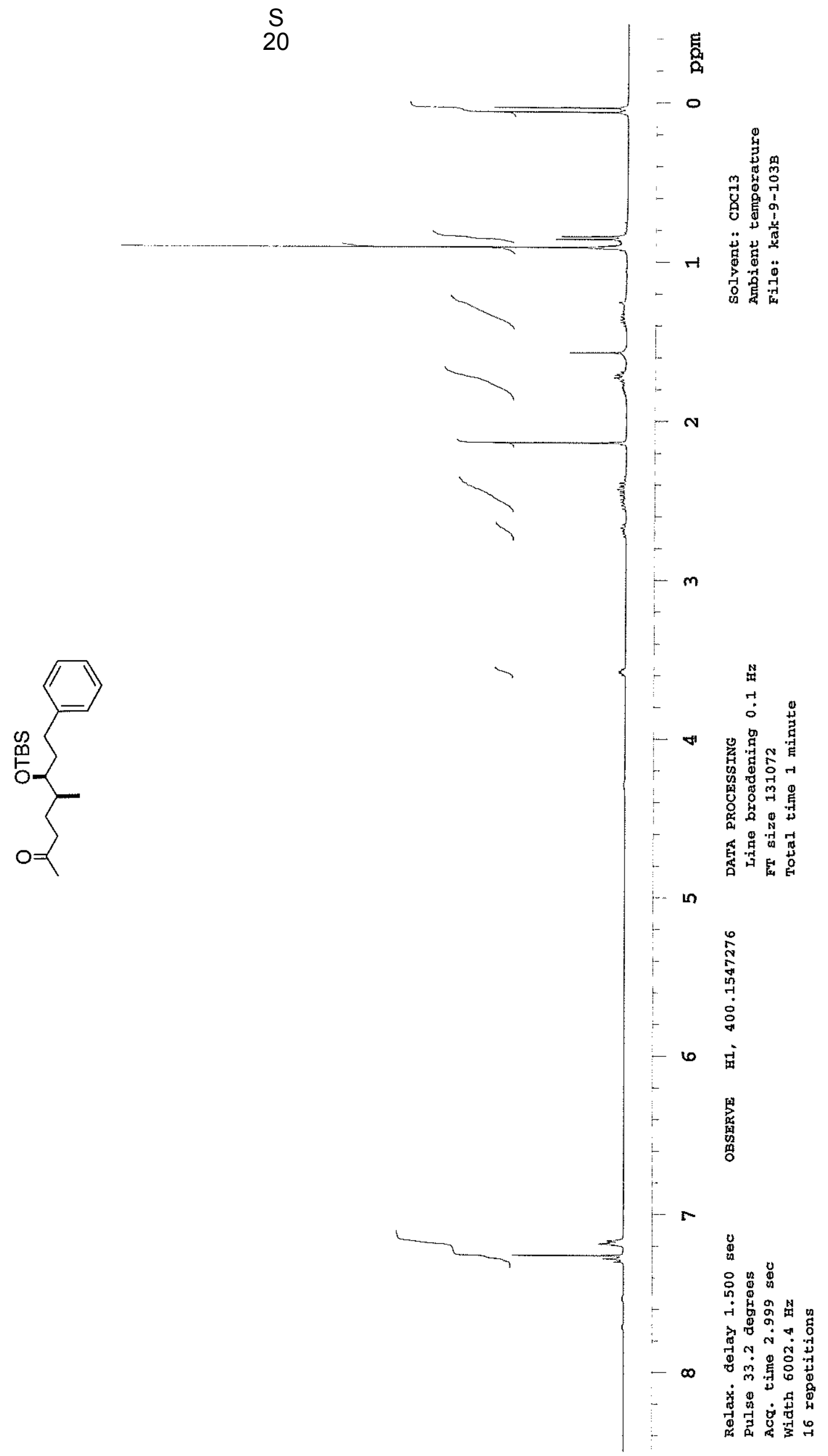




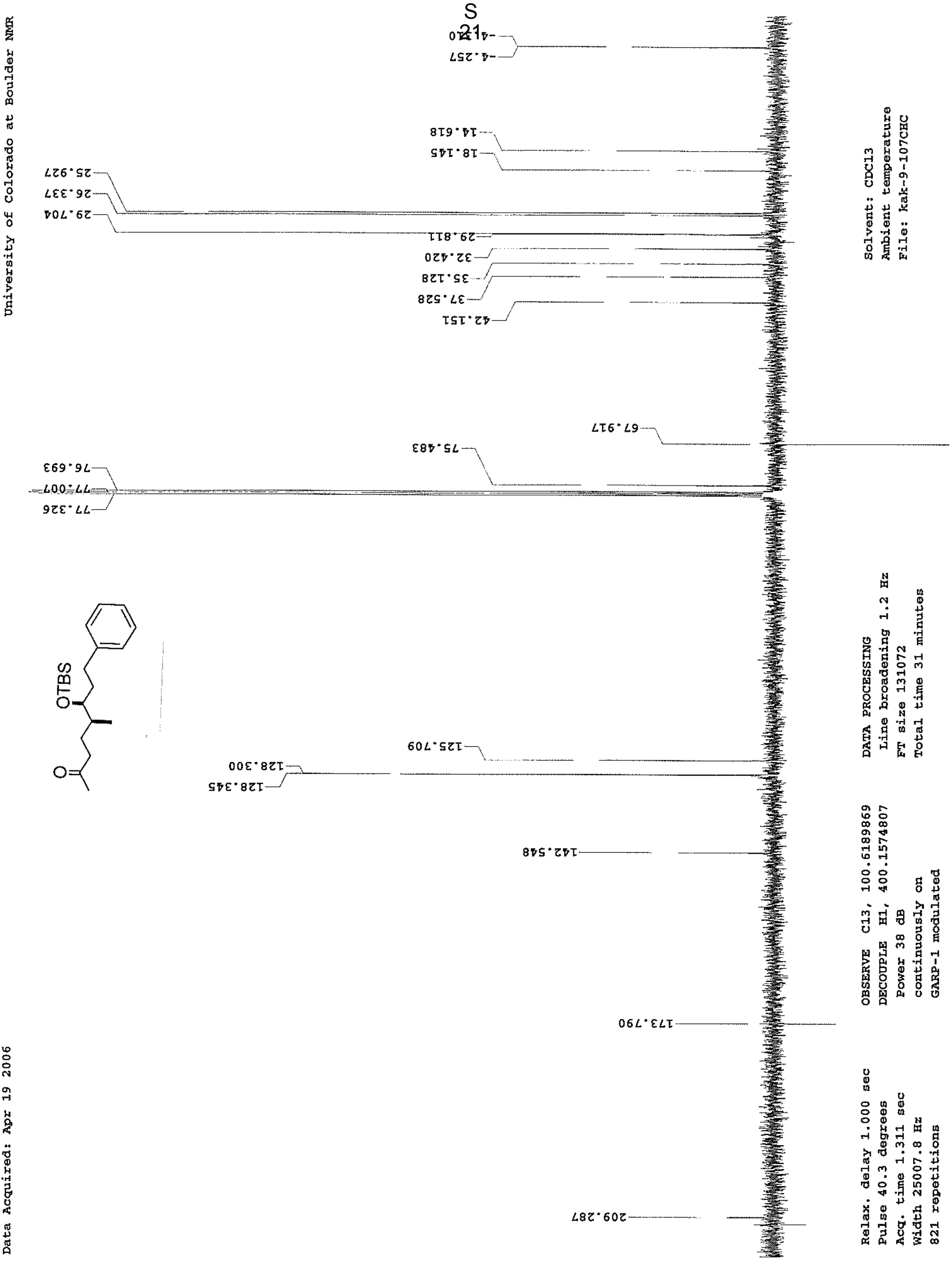




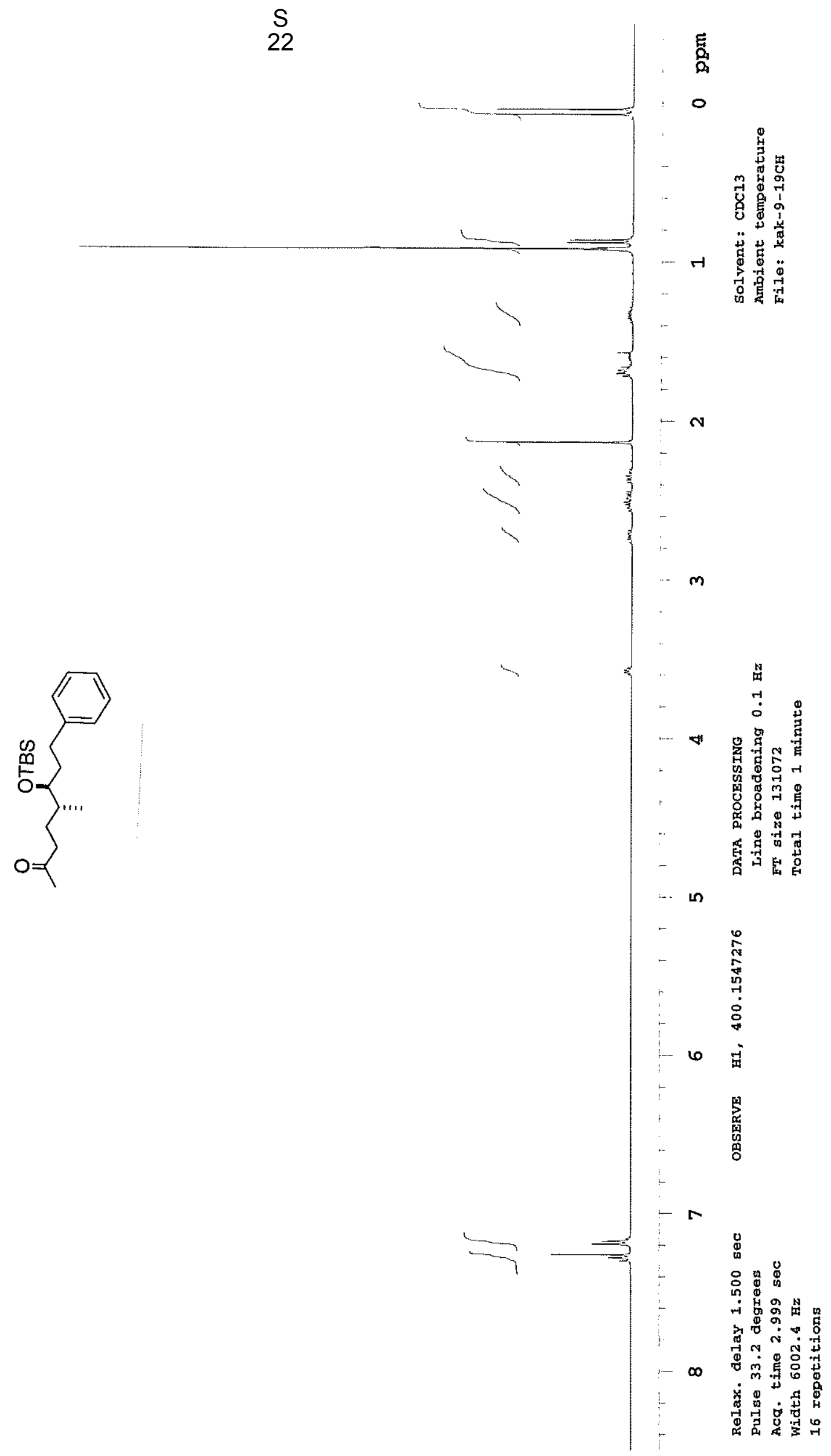




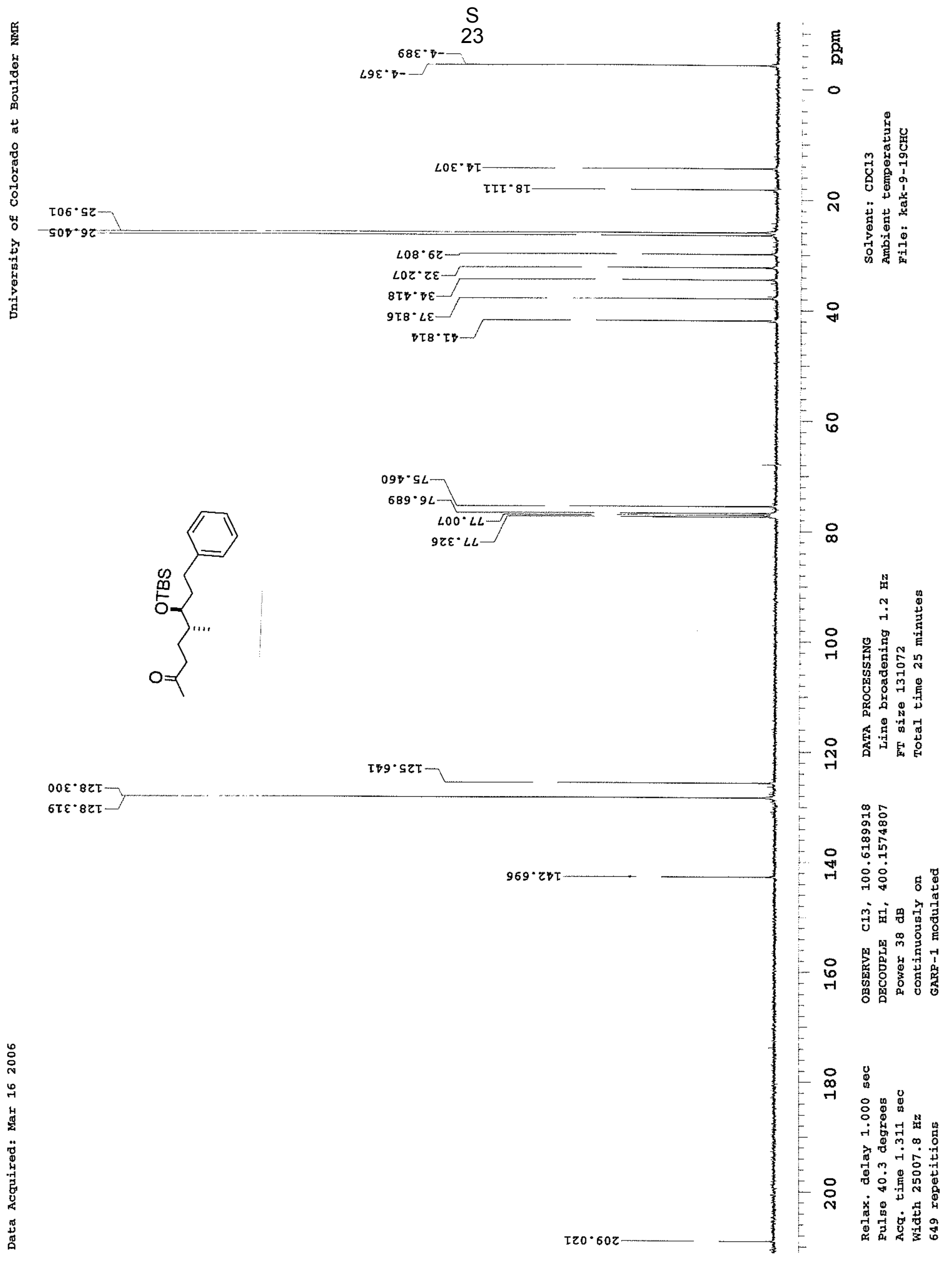




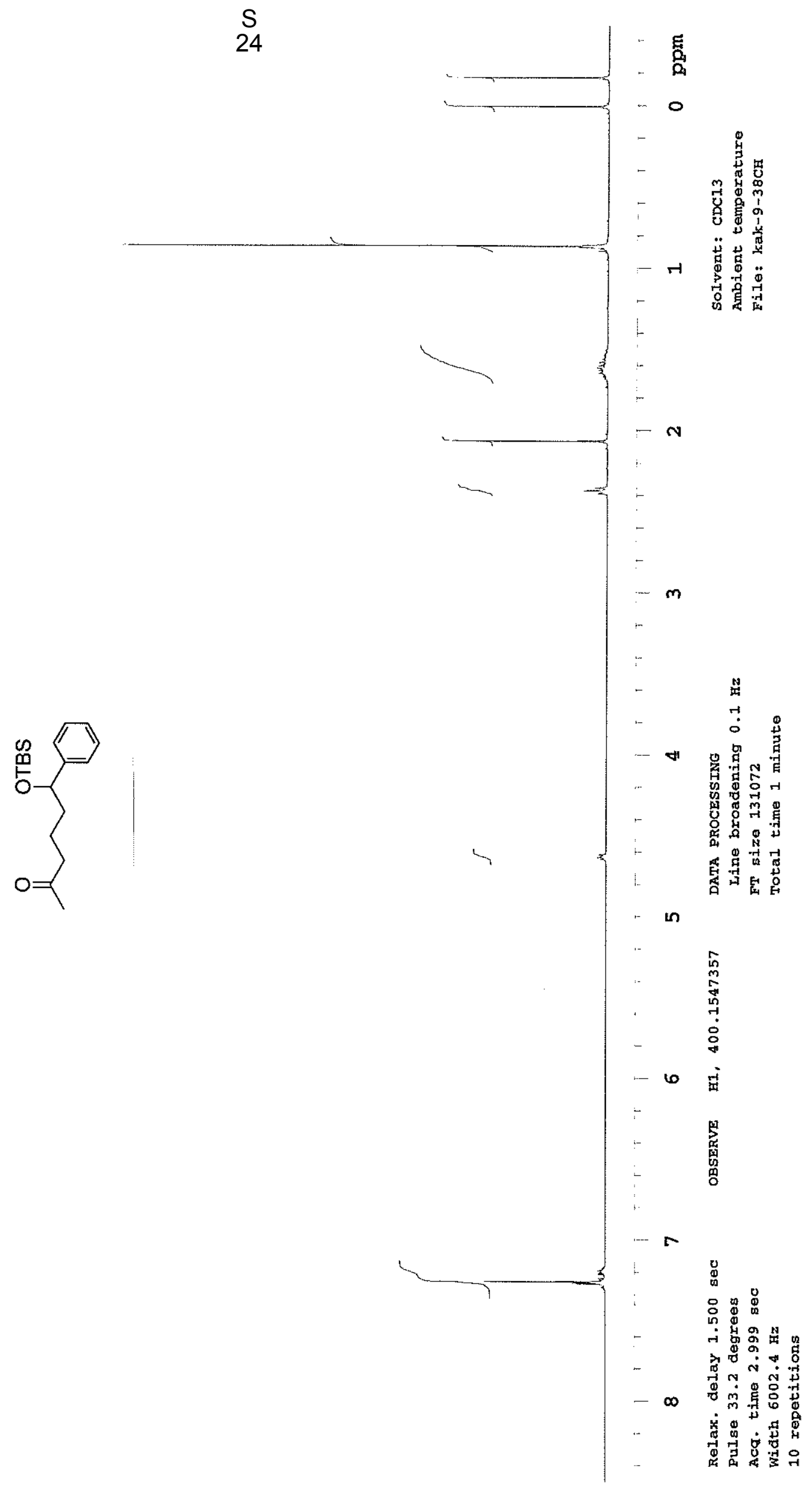




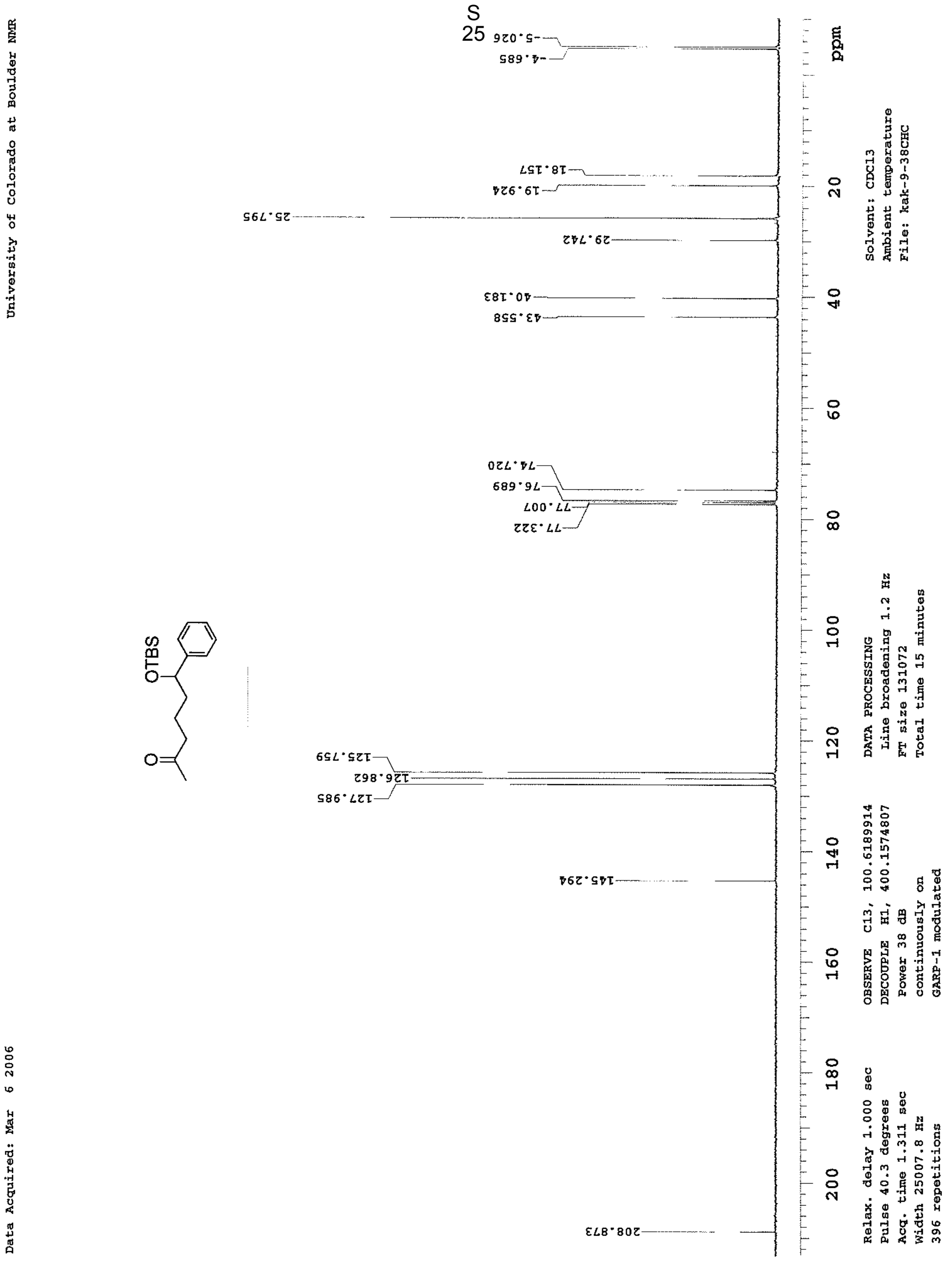



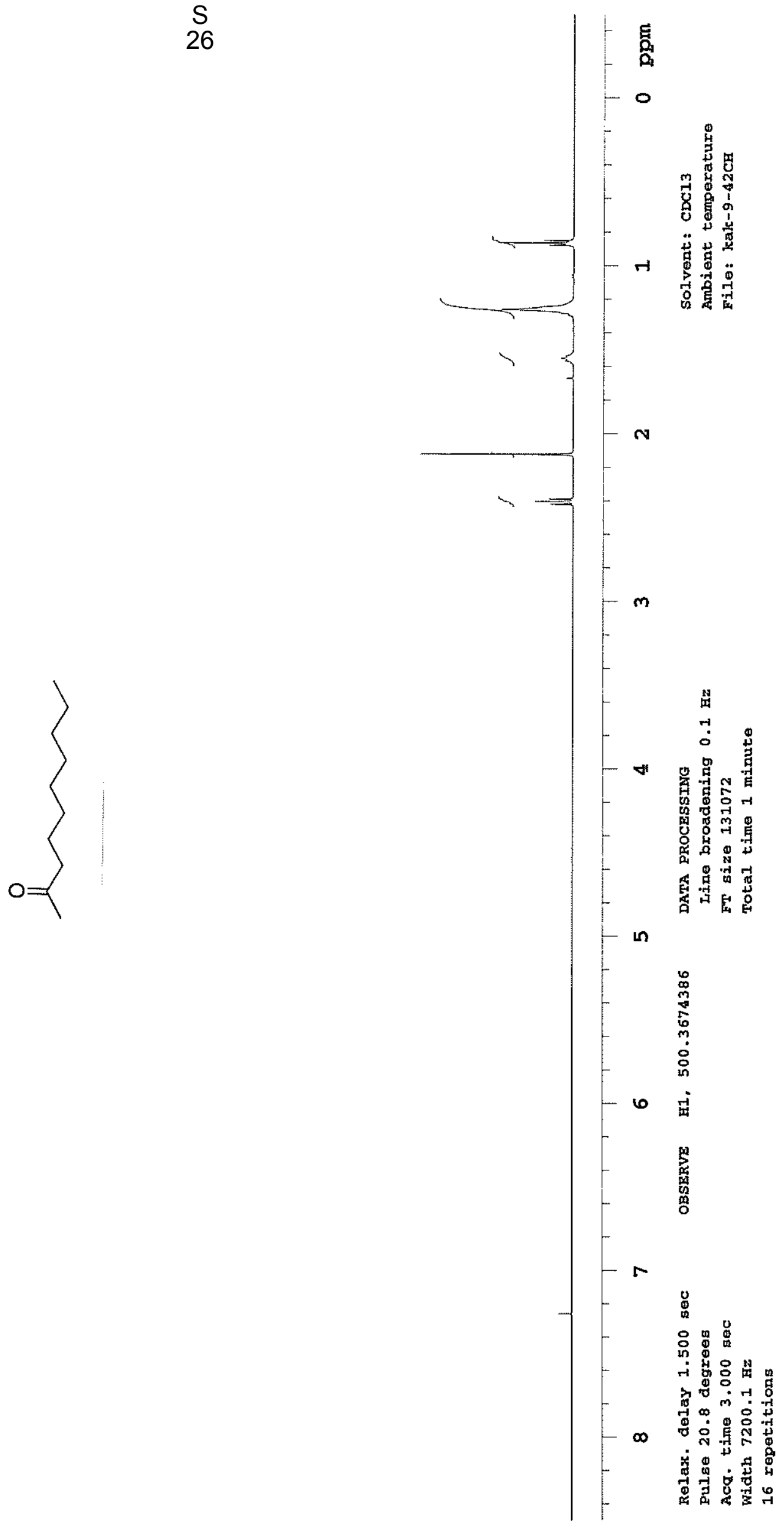


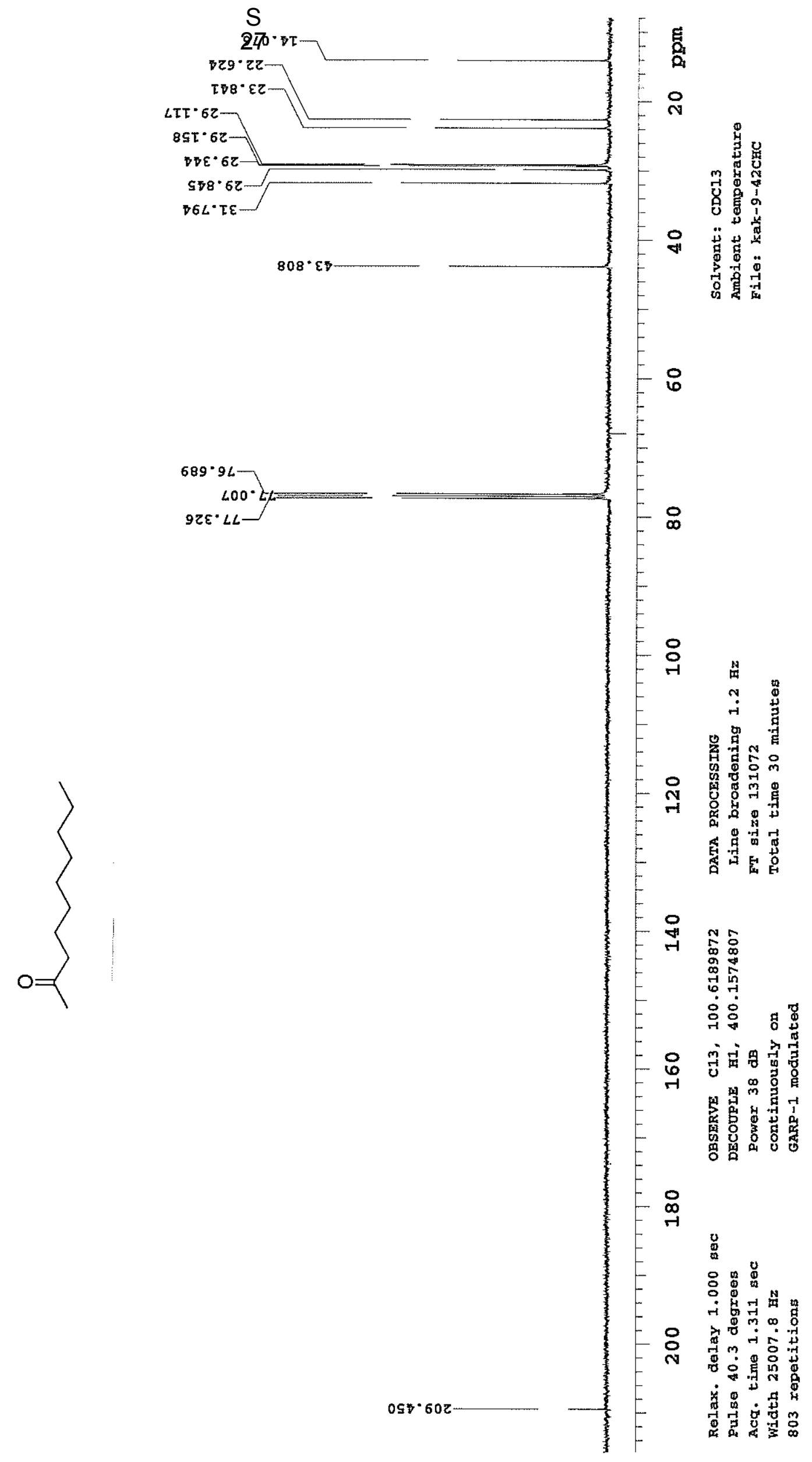

章 


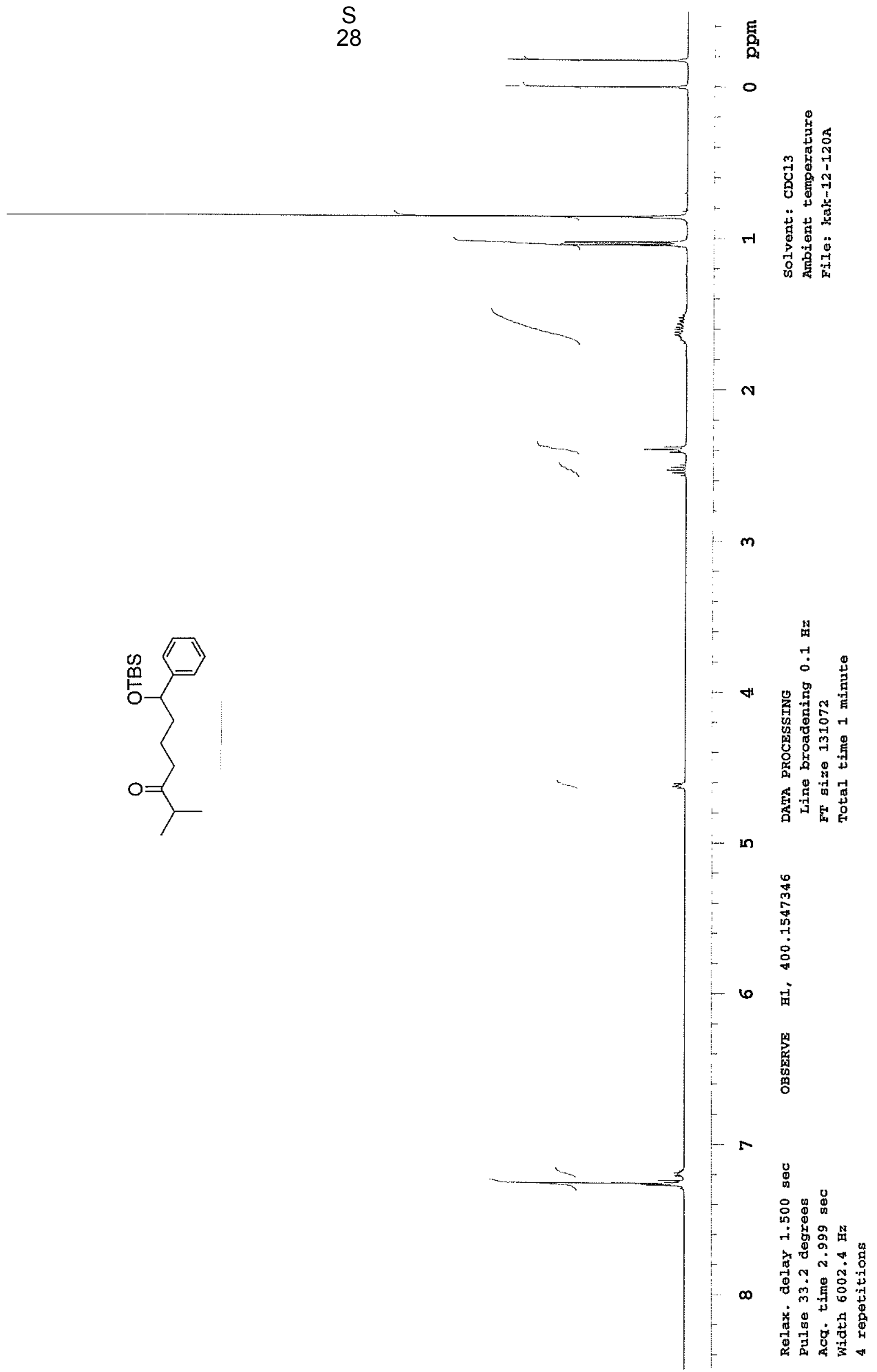




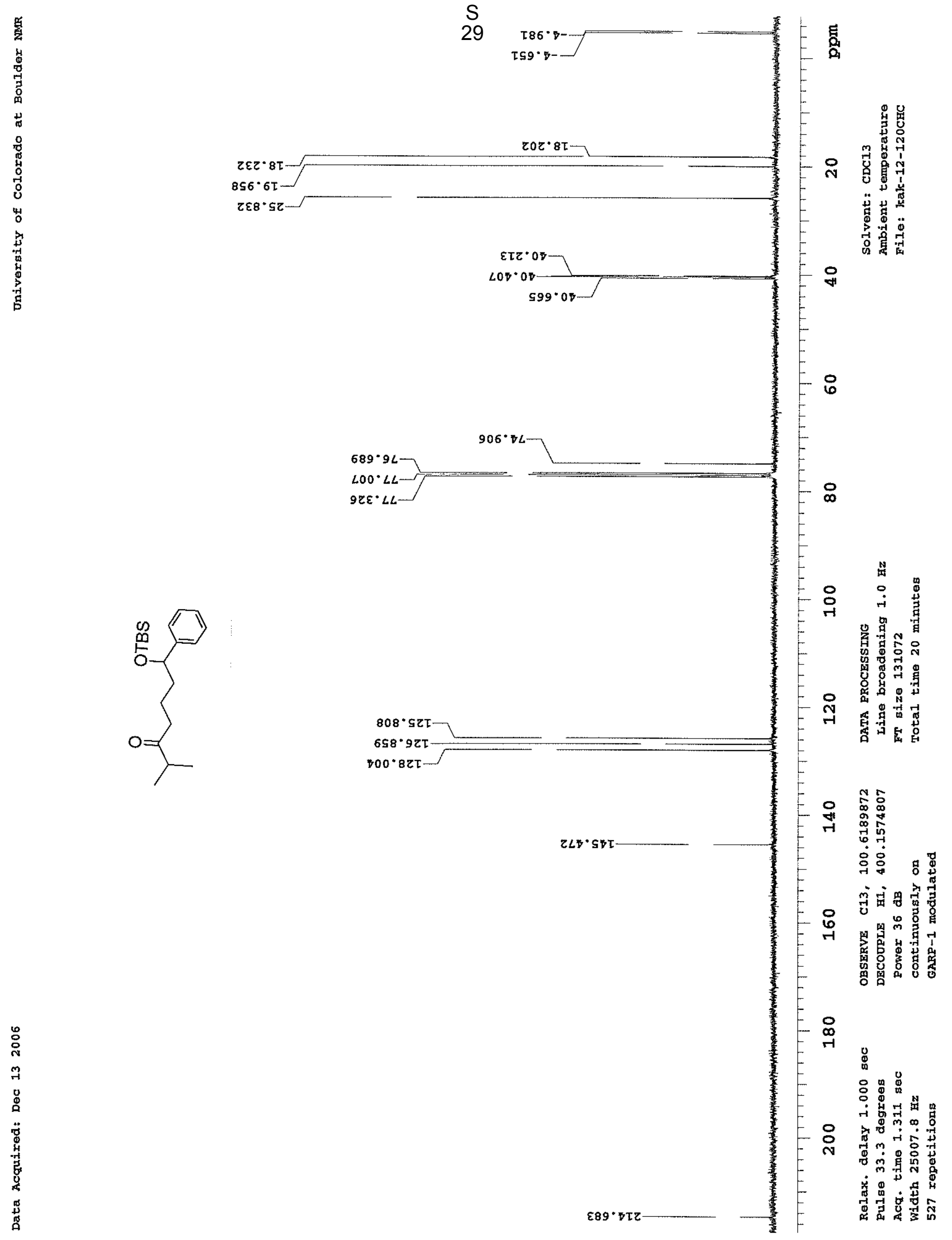




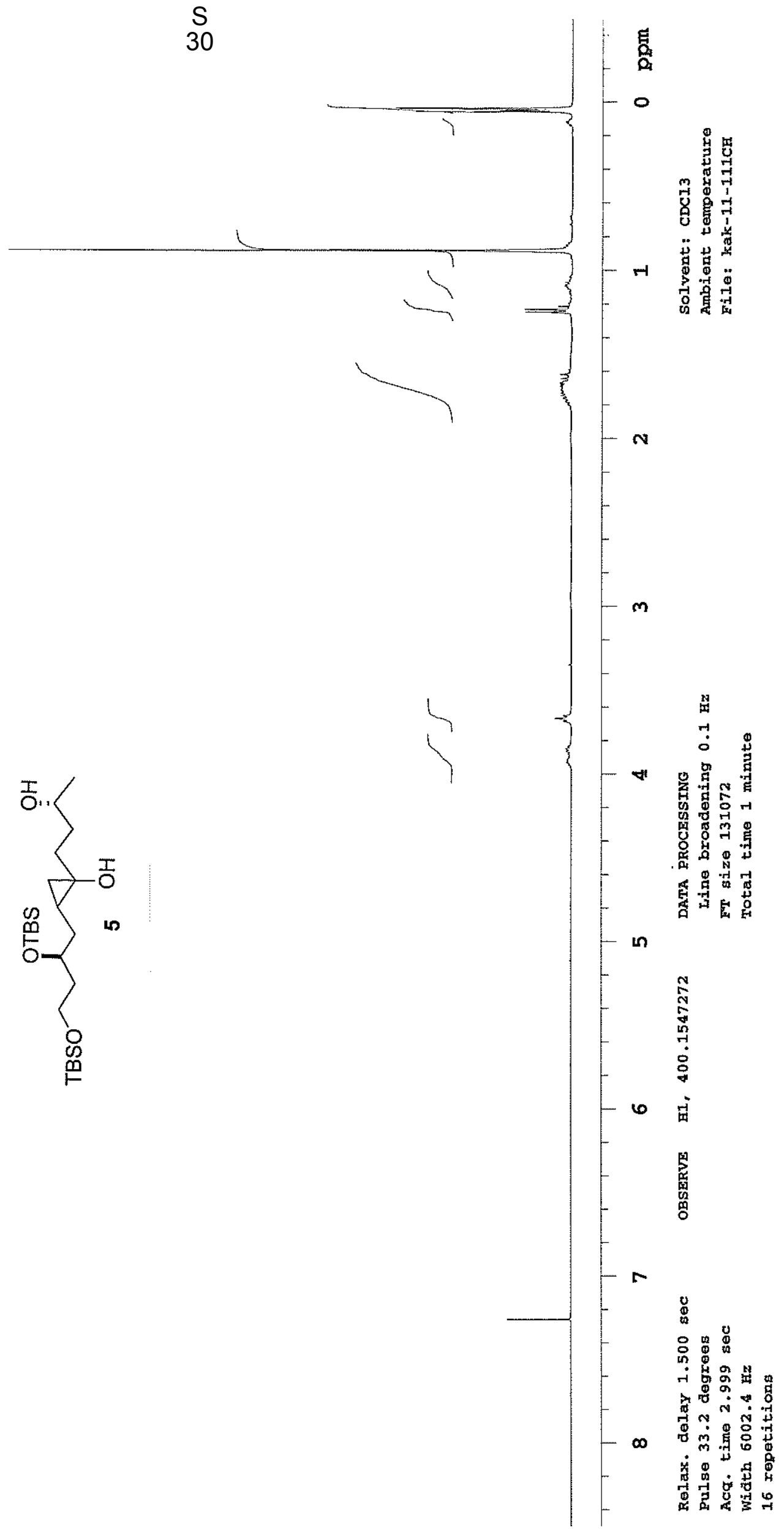



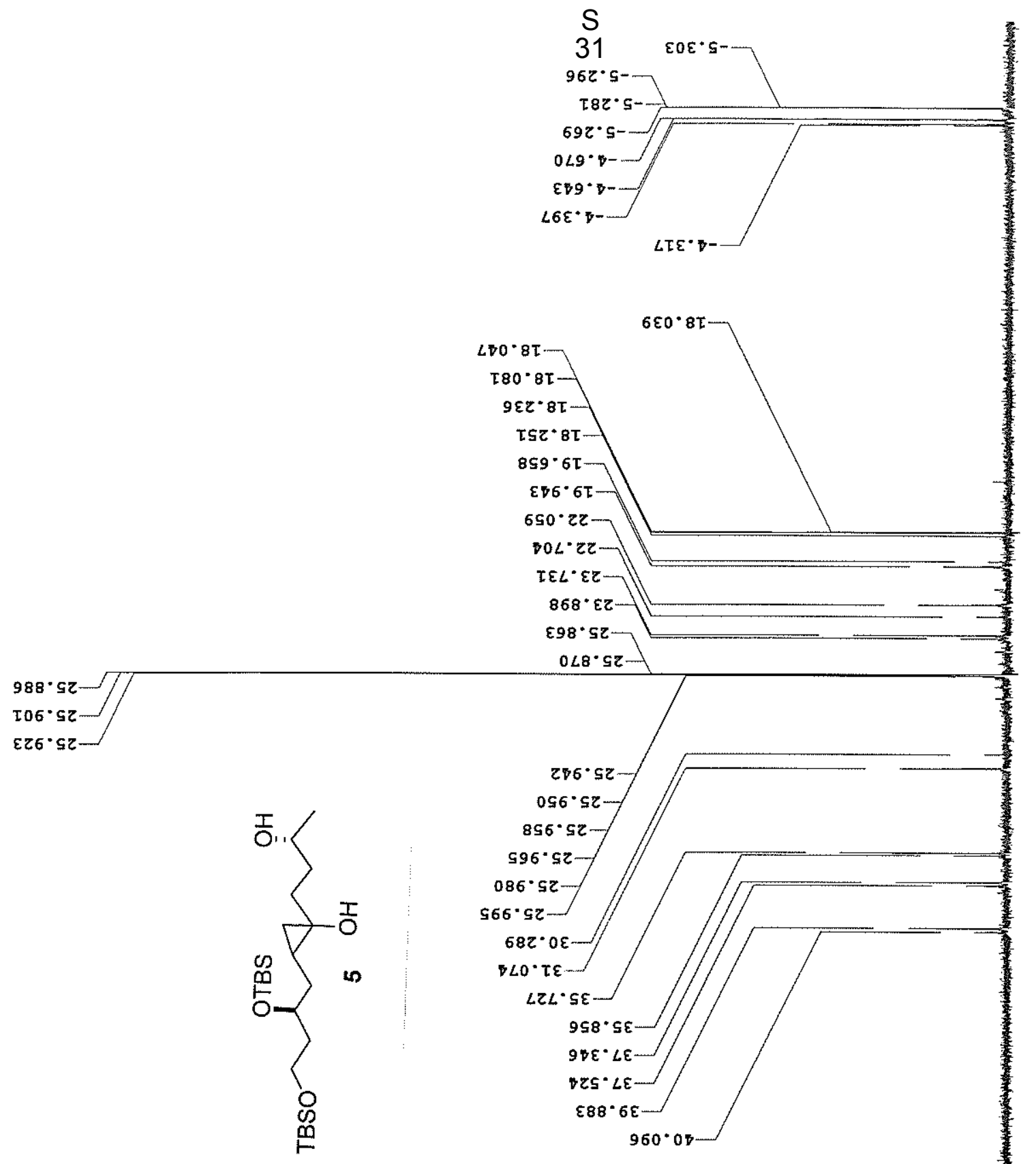

- 点

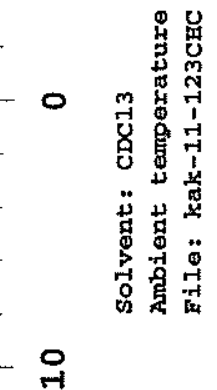

오

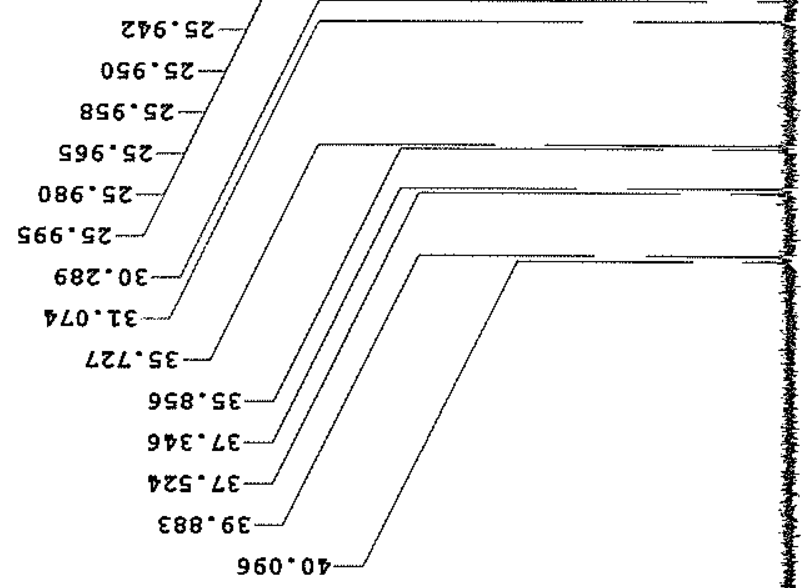

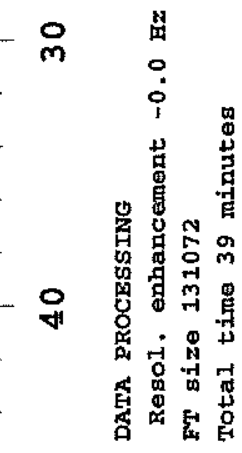

in

今ั

牙

융

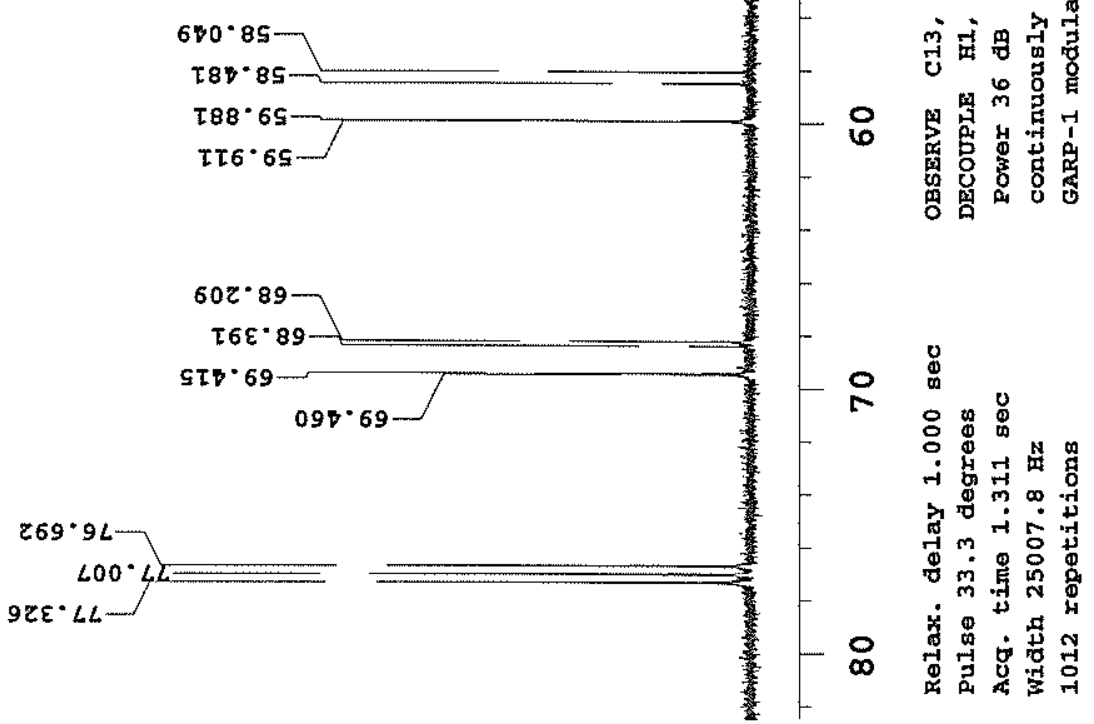

可的舀焉 


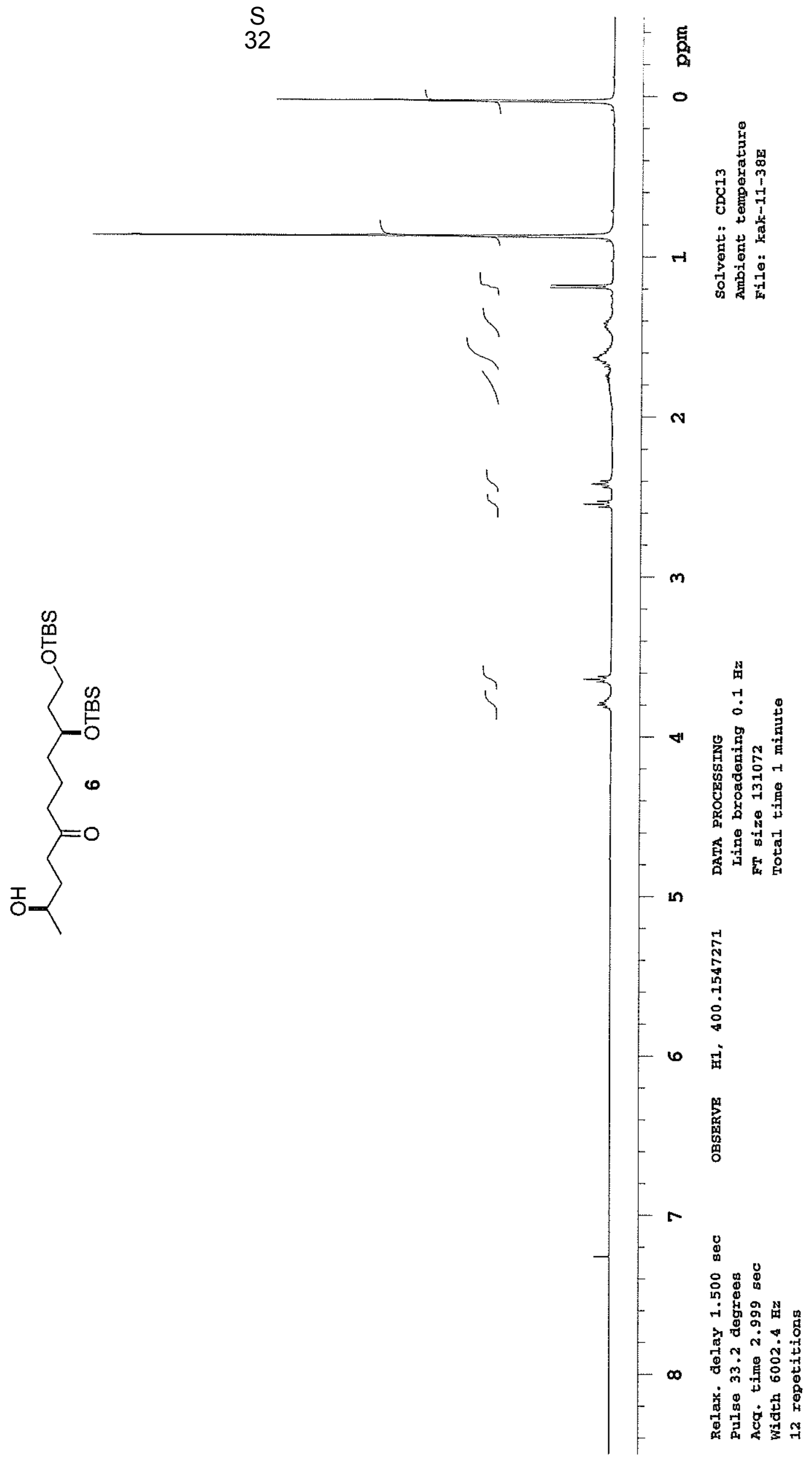




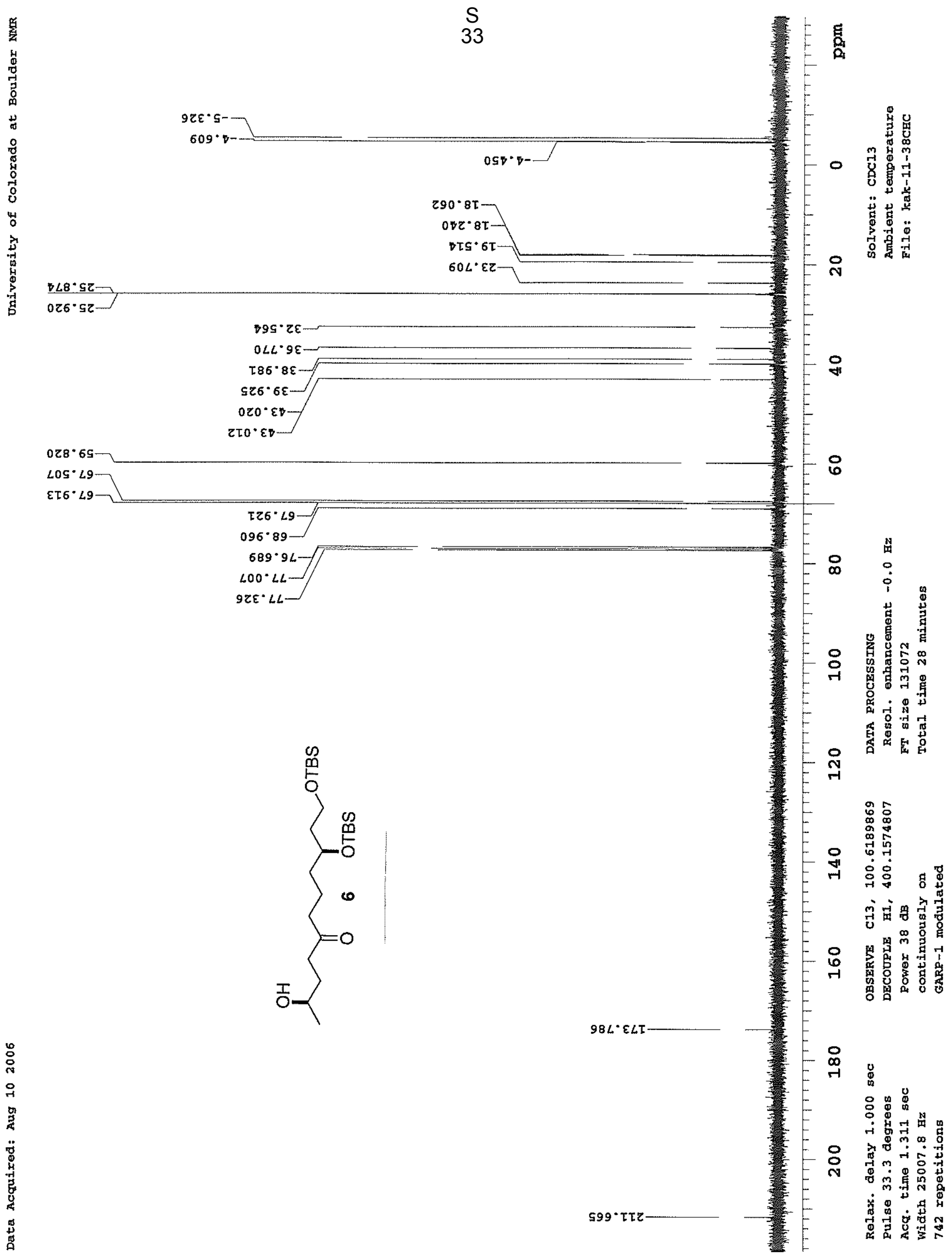



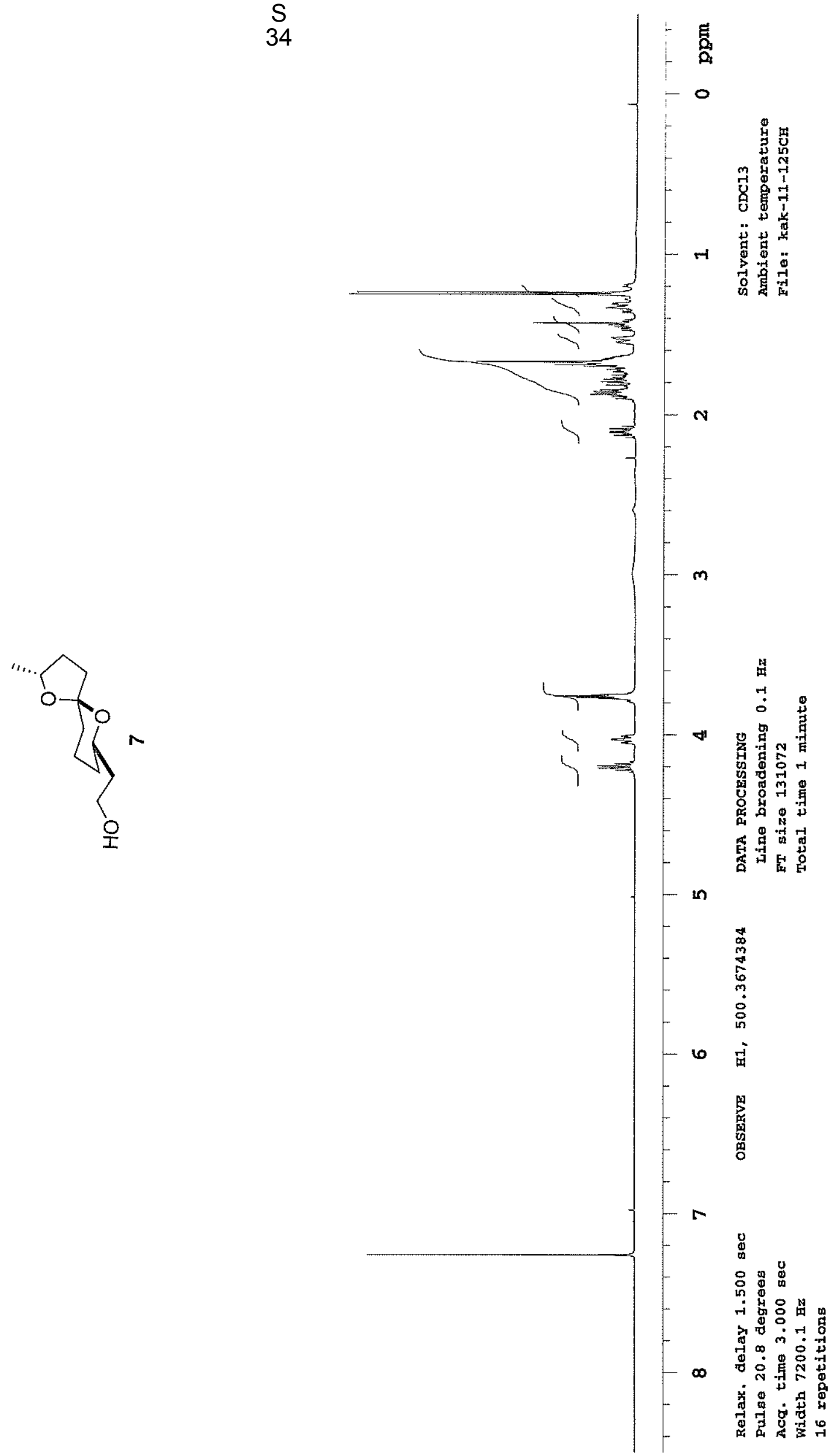


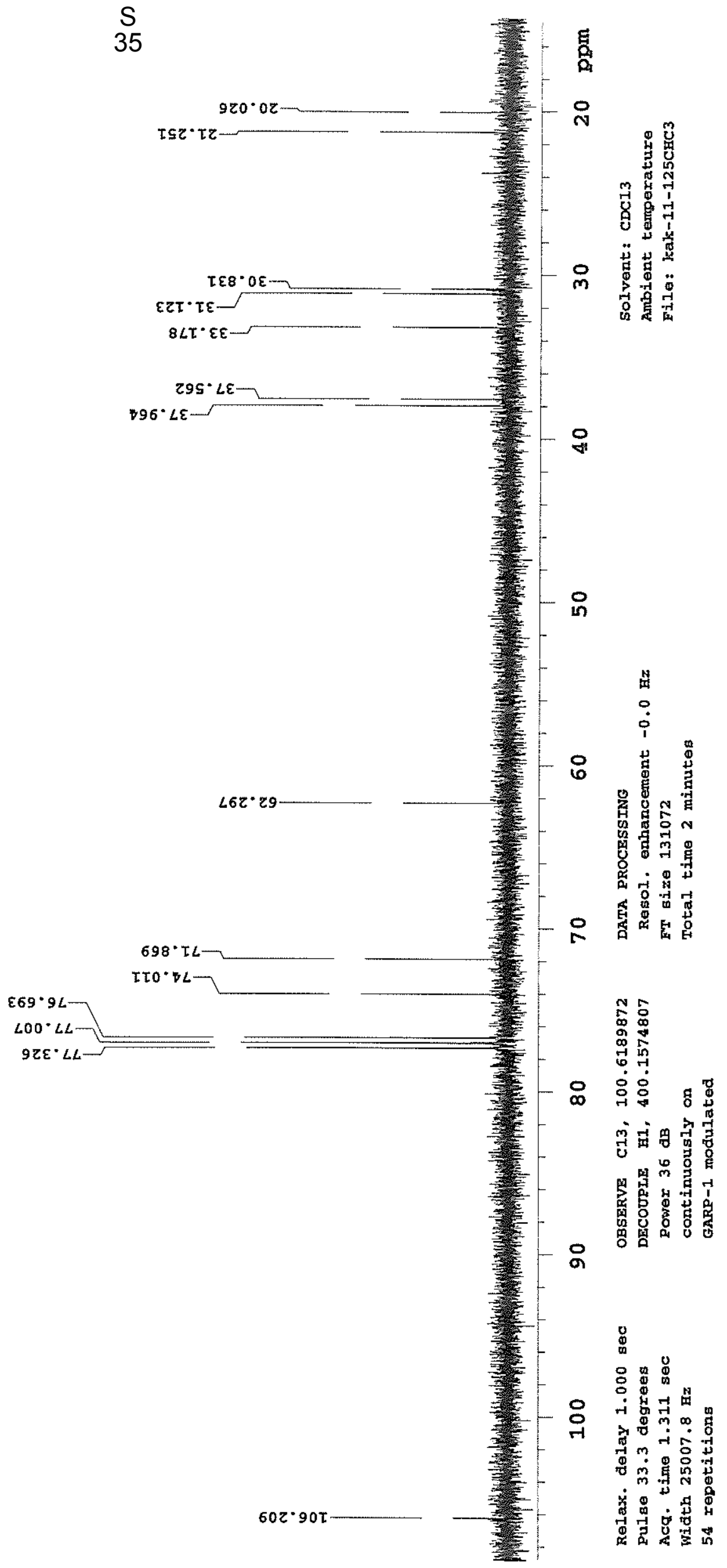



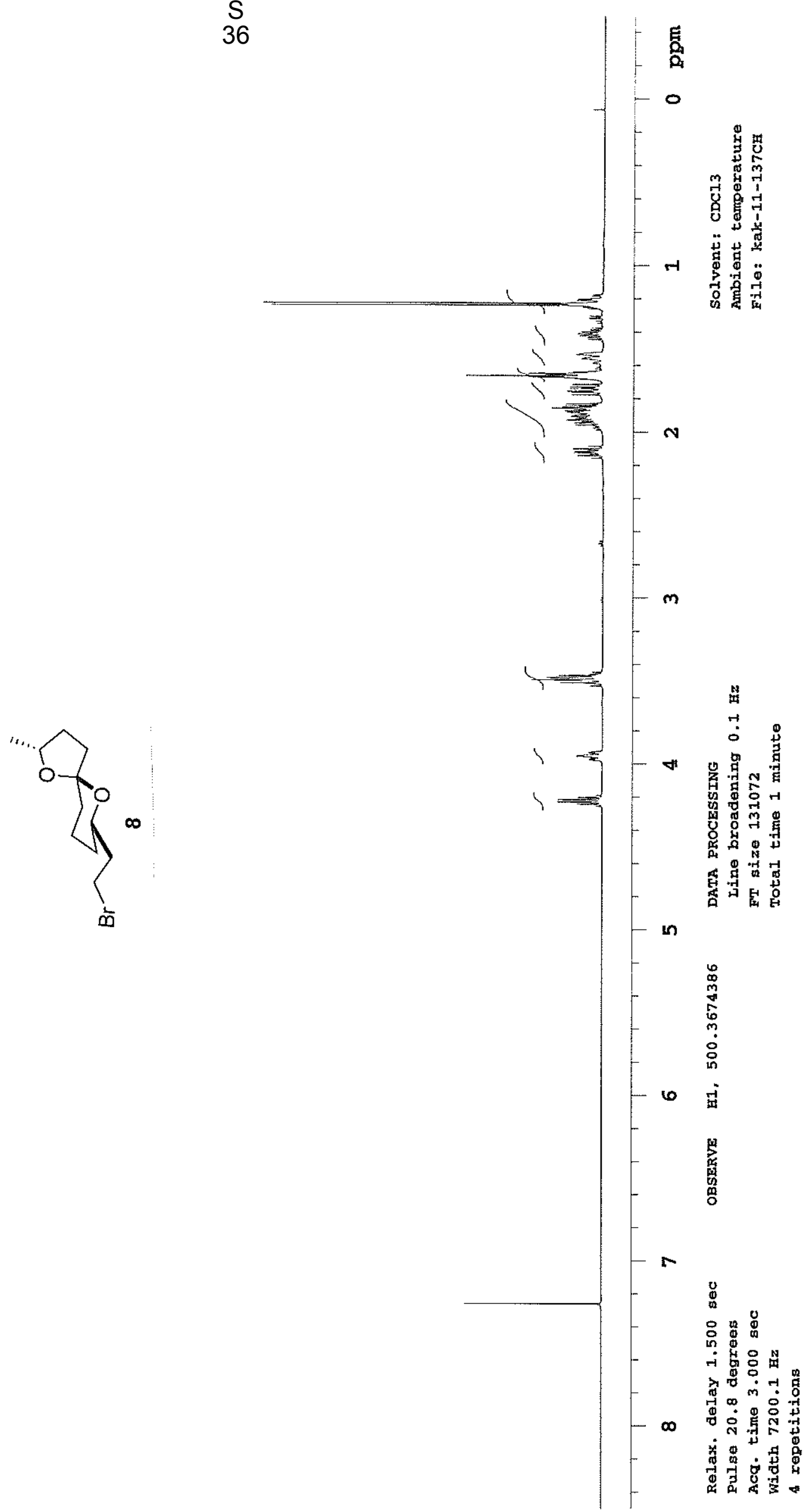

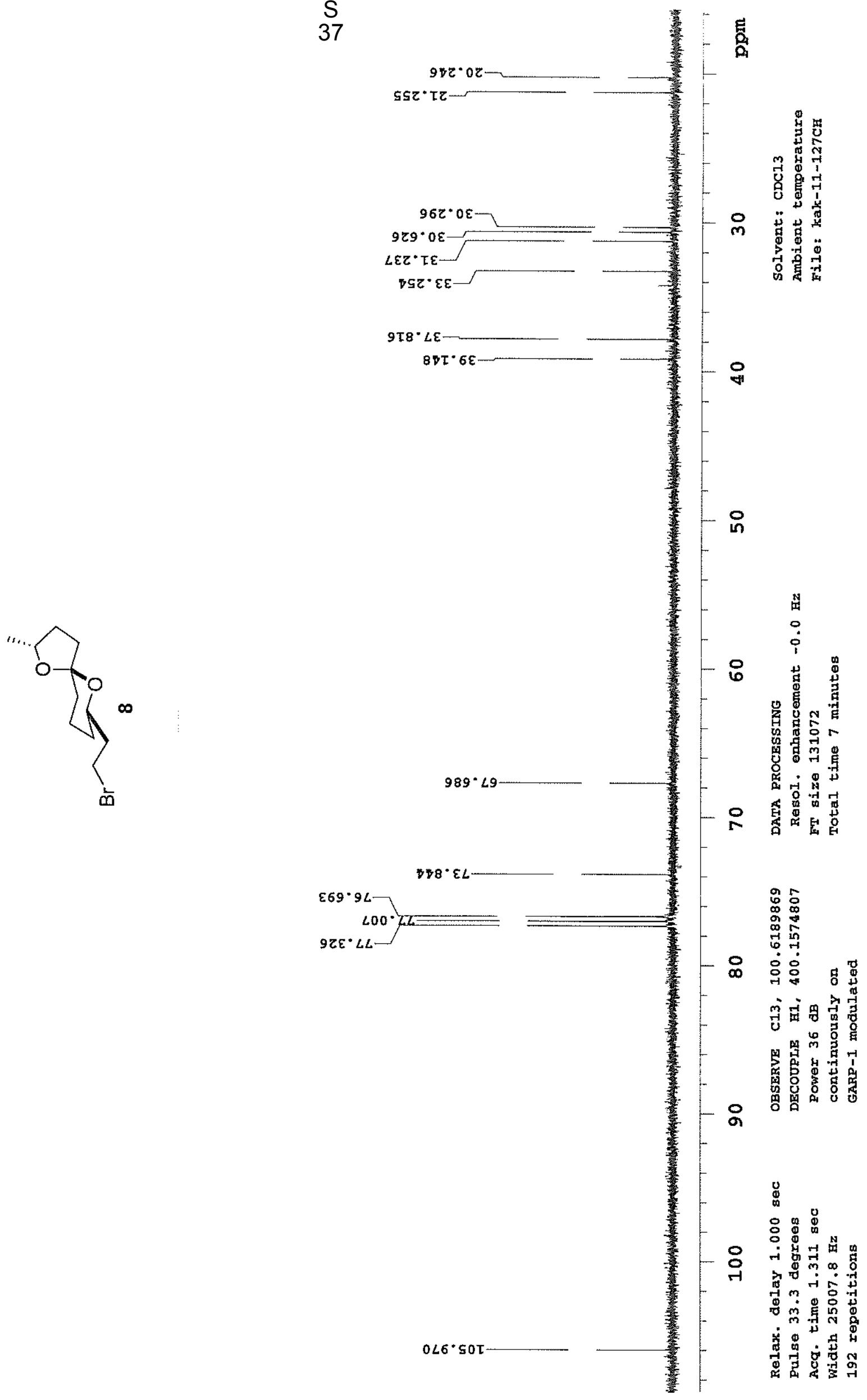


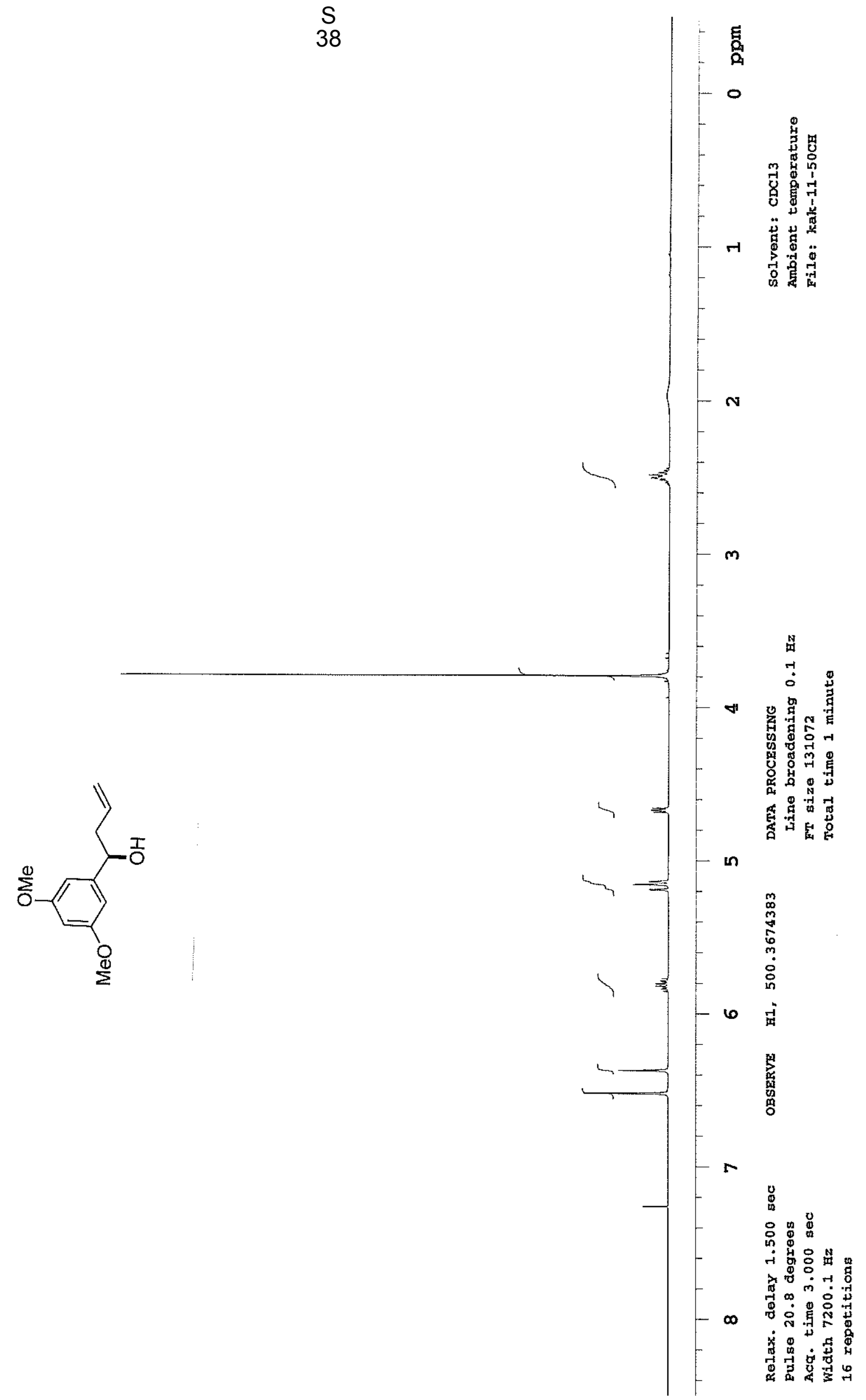




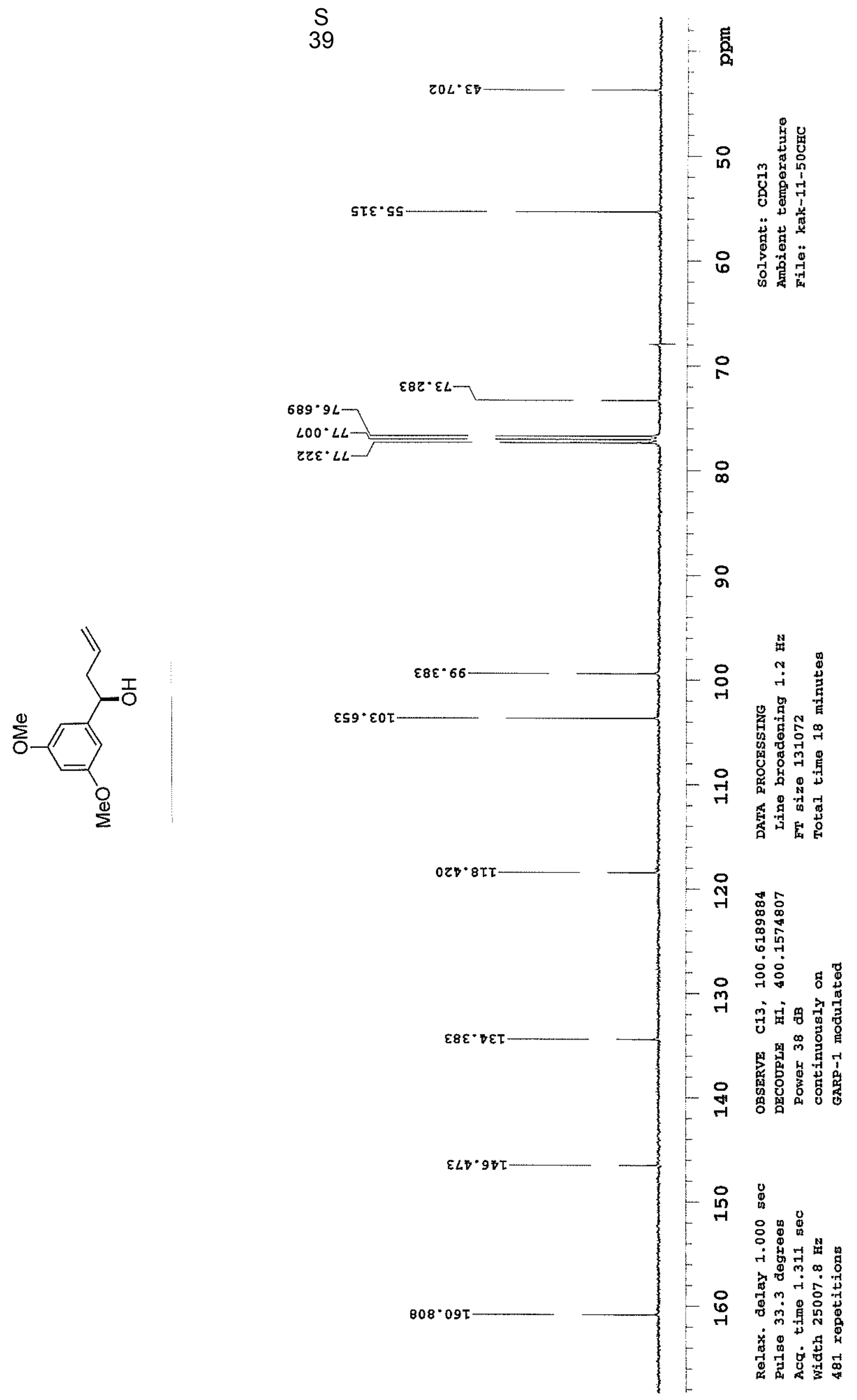




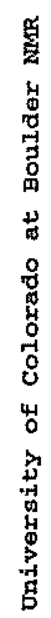

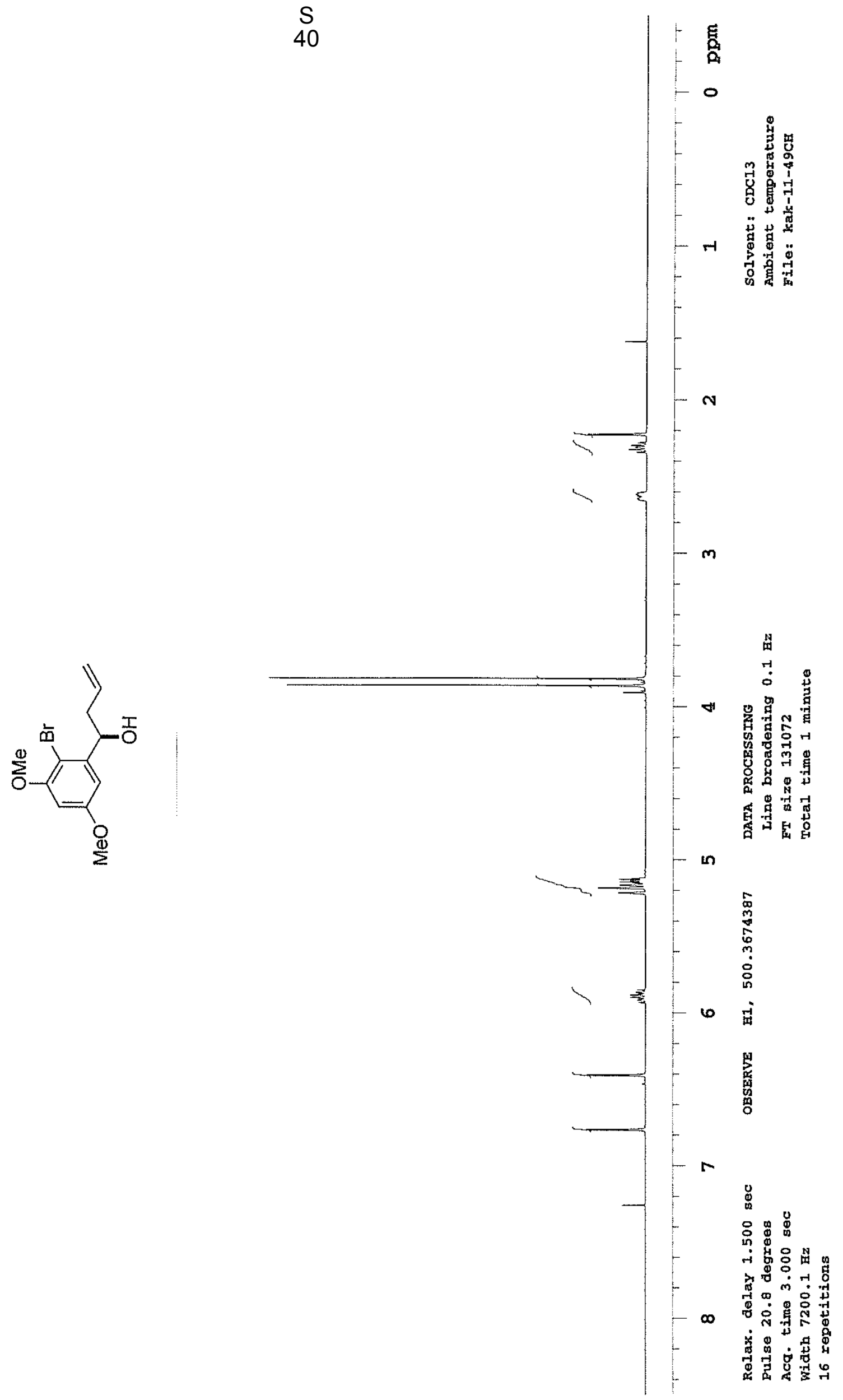




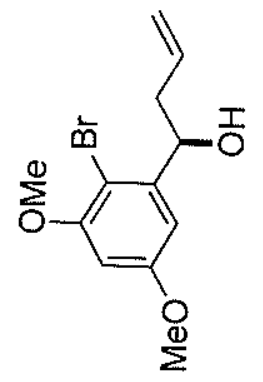

41

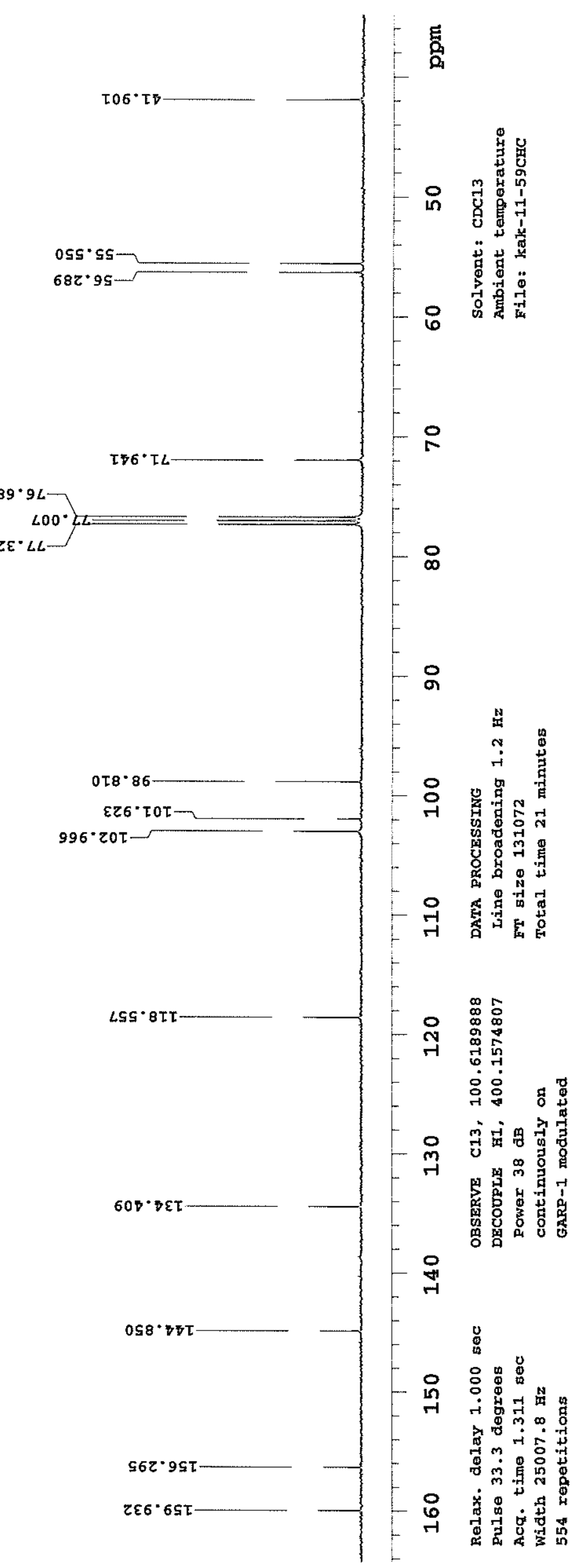



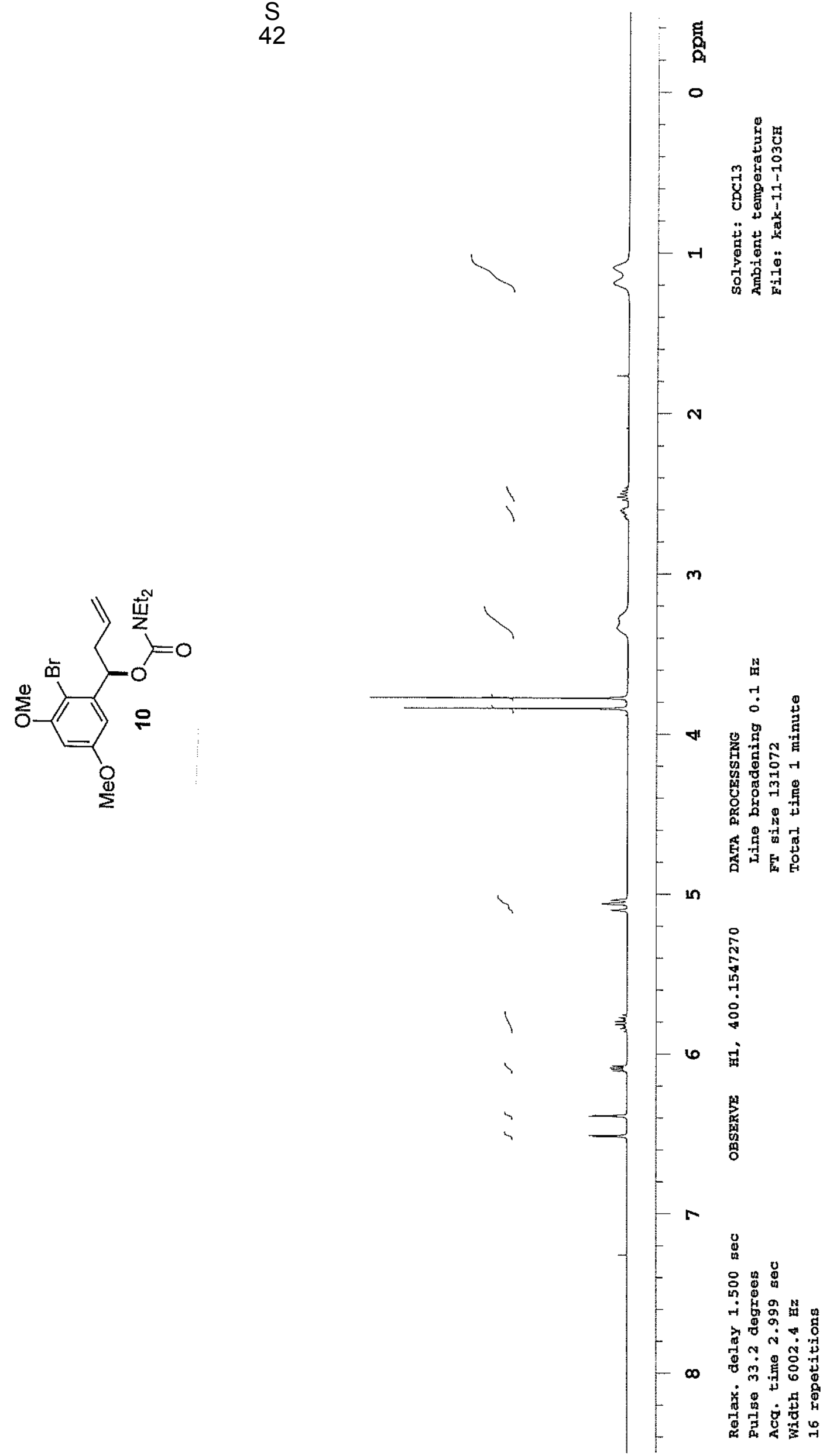


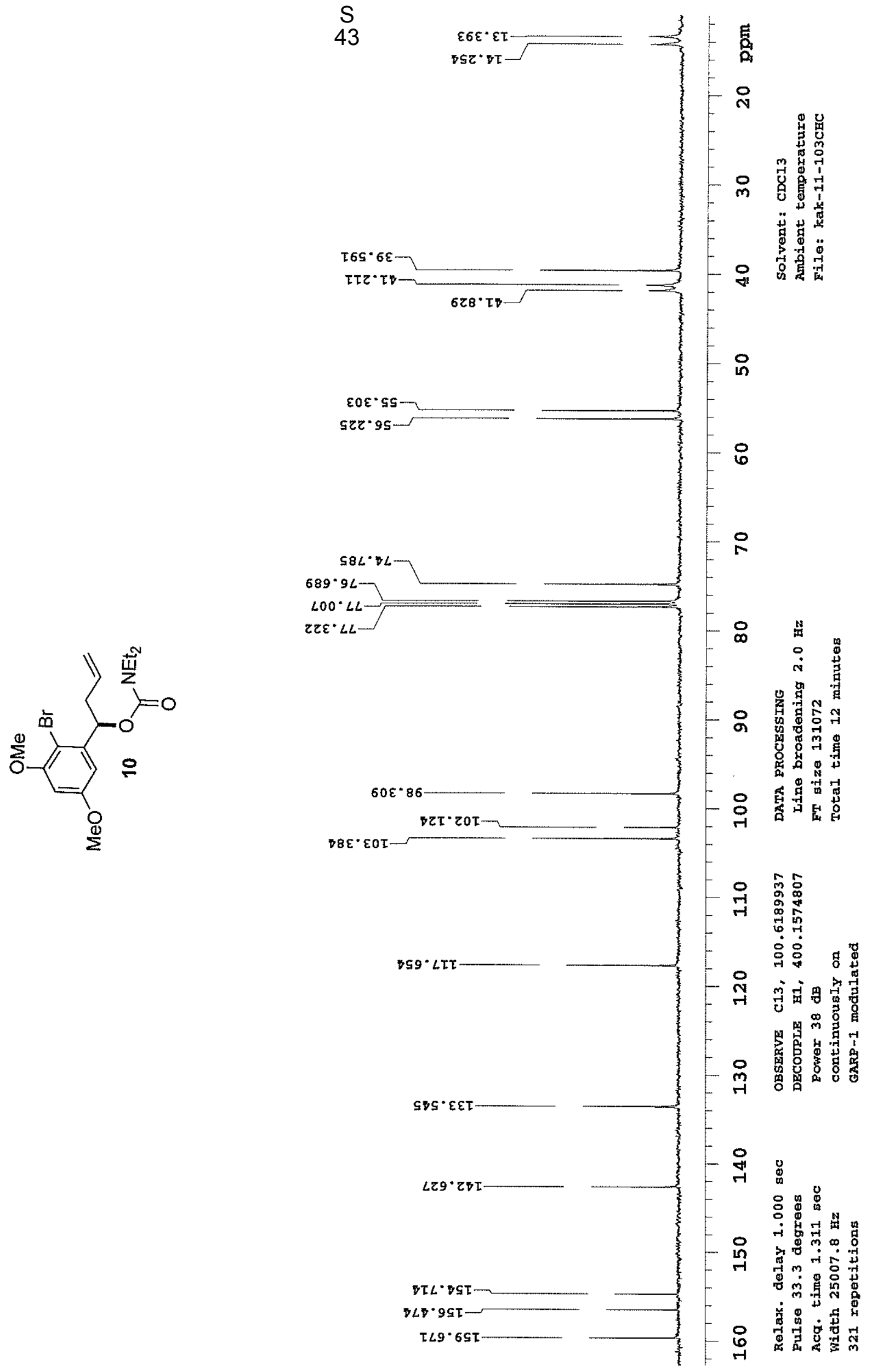



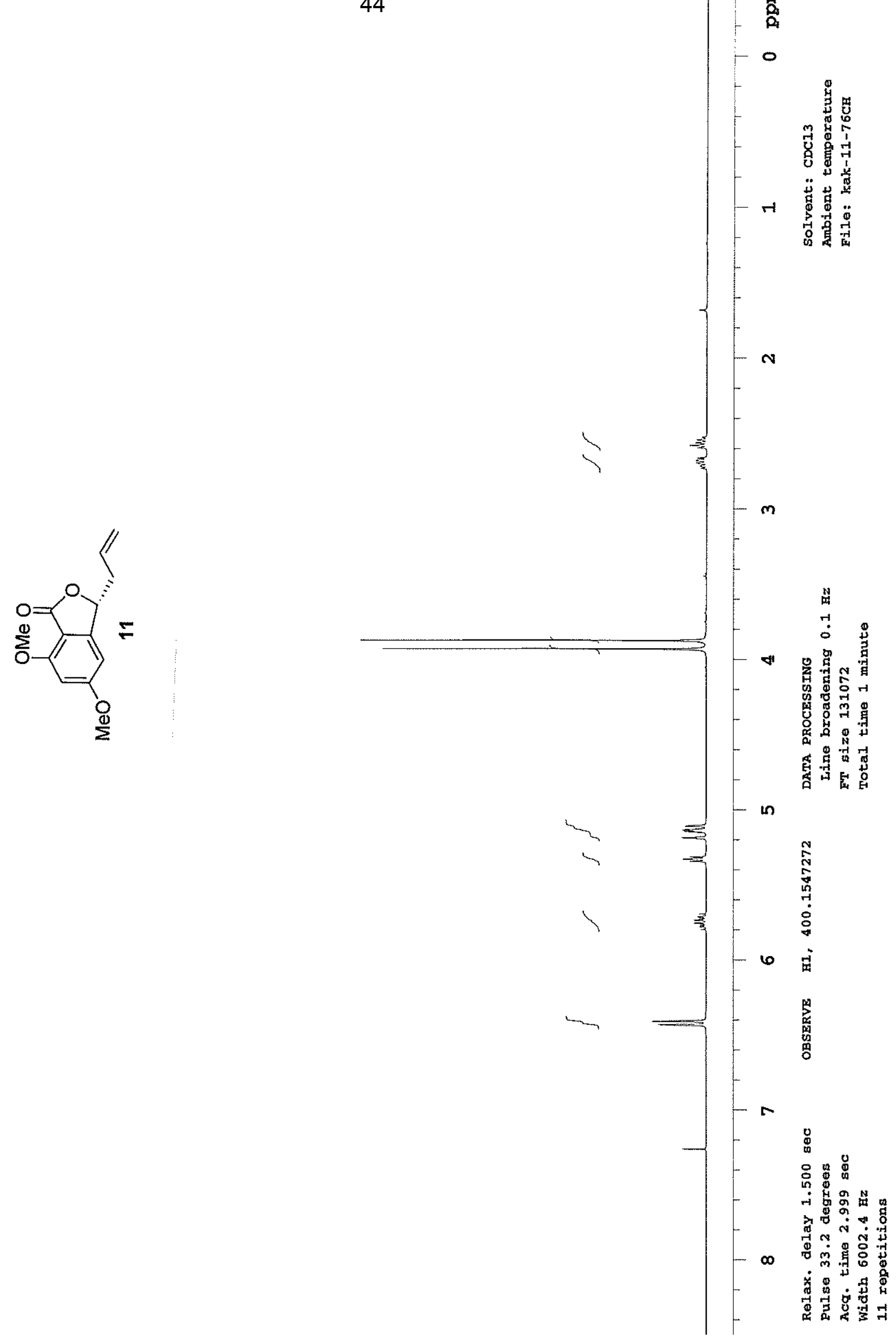


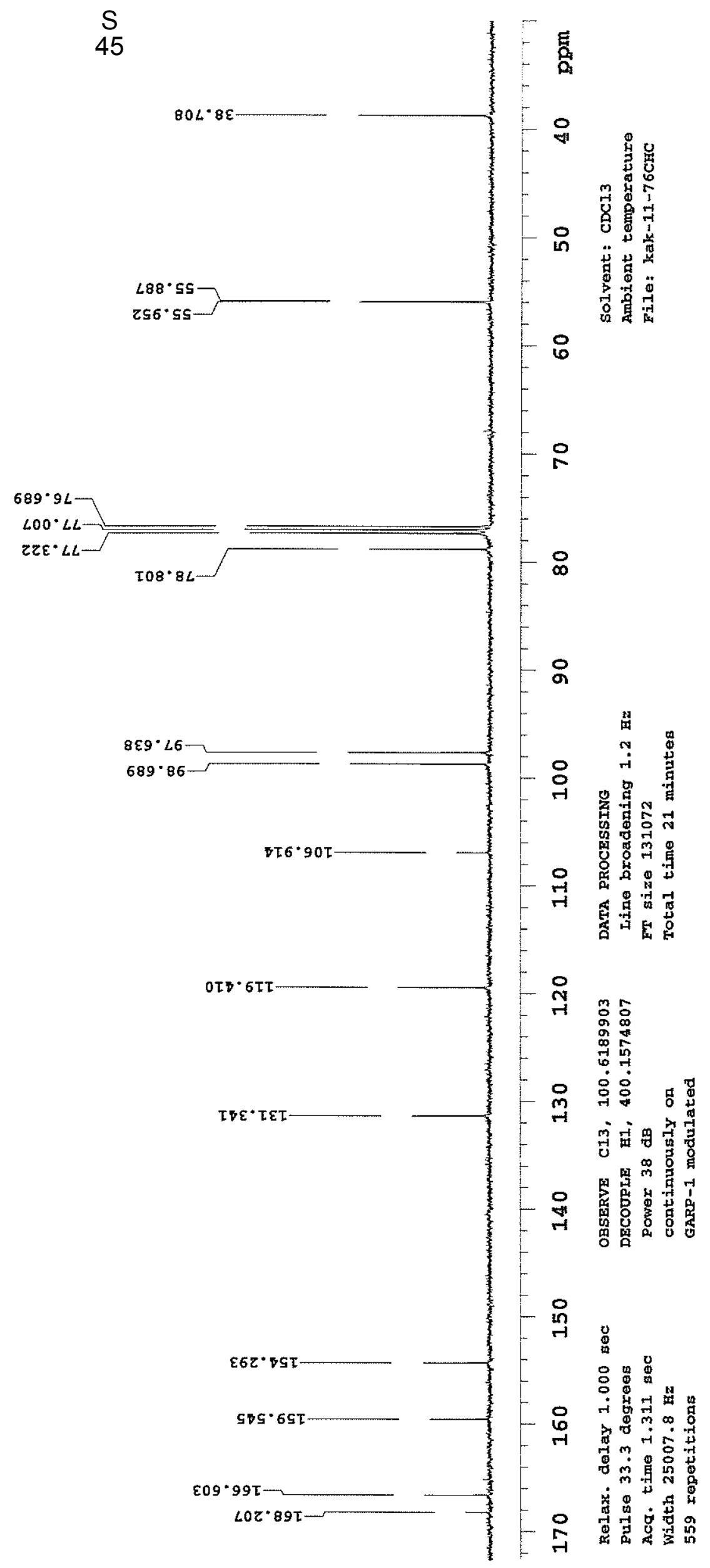




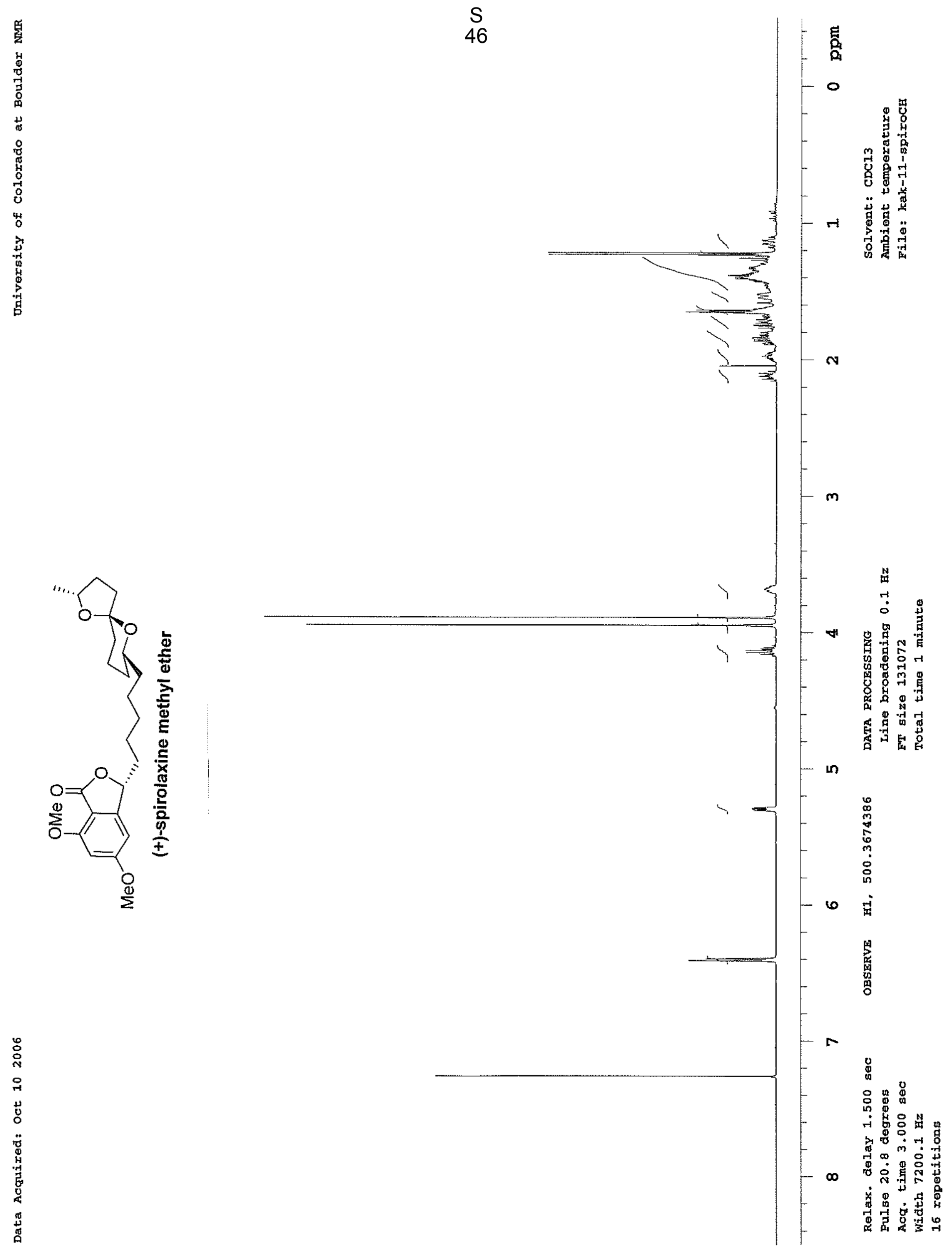




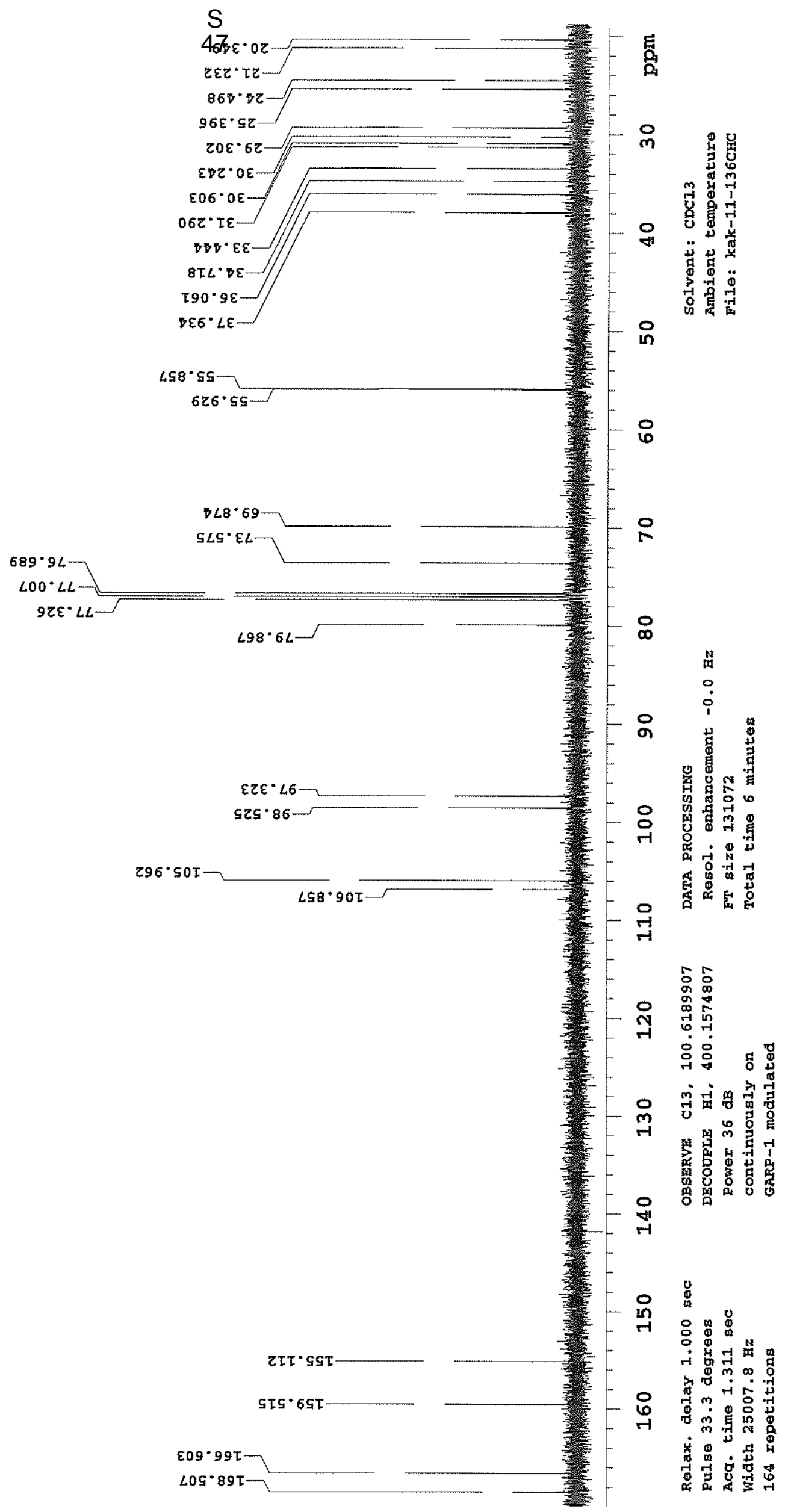

